\title{
MANY-ONE REDUCIBILITY WITHIN THE TURING DEGREES OF THE HYPERARITHMETIC SETS $H_{a}(x)\left({ }^{1}\right)$
}

BY

\author{
G. C. NELSON( ${ }^{2}$ )
}

ABSTRACT. Spector [13] has proven that the hyperarithmetic sets $H_{a}(x)$ and $H_{b}(x)$ have the same Turing degree iff $|a|=|b|$. Y. Moschovakis has proven that the sets $H_{a}(x)$ under many-one reducibility for $|a|=\gamma$ and $a \in 0$ have nontrivial reducibility properties if $\gamma$ is not of the form $a+1$ or $a+\omega$ for any ordinal $a$. In particular, he proves that there are chains of order type $\omega_{1}$ and incomparable many-one degrees within these Turing degrees. In Chapter II, we extend this result to show that any countable partially ordered set can be embedded in the many-one degrees within these Turing degrees. In Chapter III, we prove that if $\gamma$ is also not of the form $a+\omega^{2}$ for some ordinal $a$, then there is no minimal manyoone degree of the form $H_{a}(x)$ in this Turing degree, answering a question of $Y$. Moschovakis posed in [8]. In fact, we prove that given $H_{a}(x)$ there are $H_{b}(x)$ and $H_{c}(x)$ both many-one reducible to $H_{a}(x)$ with incomparable many-one degrees, $|a|=|b|=|c|=\gamma$.

\section{CHAPTER I. PRELIMINARIES}

For the most part we adopt the terminology and notation as introduced by Kleene in [4], [5], and [6]. For definiteness, our Gödel numbering of the partial recursive functions of $n$-variables will be the particular one given in [4]. Also, we use freely the function $U(z)$ and the $T$-predicates of Kleene [4].

We assume familiarity with the notions of many-one (one-one) reducibility of $A$ to $B$ and denote this by $A \leq_{m} B\left(A \leq_{1} B\right)$ [10] and [11]. Similarly, we write $A \leq_{T} B$ if $A$ is Turing reducible to $B$ [7], i.e., $A$ is recursive in $B$ [5]. Degrees will refer to the equivalence classes of sets indistinguishable under a specified

Presented to the Society, September 23, 1968 under the title Reducibility orderings of the hyperarithmetic predicates; received by the editors June 25, 1971 and, in revised form, June 1, 1973.

AMS (MOS) subject classifications (1970). Primary 02F 27, 02F 35.

Key words and phrases. Constructive ordinal notations, many-one degrees, Turing degrees, recursively majorized.

(1) The results of this paper are contained in the author's Ph.D. thesis at Case Western Reserve University, June 1968, written under the direction of C. F. Kent.

(2) The author expresses his appreciation to the referee for his corrections and suggestions. 
reducibility and we denote, for example, the many-one degrees of $A$ by $[A]_{m}$.

We let $\mathcal{O}$ denote the set of constructive ordinal notations of Kleene [3] or [6]. As in [8], if $3 \cdot 5^{y} \in \mathcal{O}$, then we denote $\{y\}\left(n_{0}\right)$ by $y_{n^{*}}$. For $b$ in $\mathcal{O}$, there is associated a unique ordinal denoted by $|b|$ which is defined inductively on $\mathcal{O}$ as $|1|=0,\left|2^{y}\right|=|y|+1$, and $\left|3 \cdot 5^{y}\right|=\lim _{n \rightarrow \infty}\left|y_{n}\right|$. We assume familiarity with the relation $a<_{0} b$ for $a, b$ in $\mathcal{O}$.

The next two theorems are proven in [3] and are basic to our constructions.

Theorem 1.1. There is a primitive recursive function enm $(b, m)$ such that, if $b \in \mathcal{O}$ and $|b| \geq \omega$, then enm $(b, 0)$, enm $(b, 1), \ldots$ is an enumeration witbout repetitions of all $a \in \mathcal{O}$ sucb that $a<_{0} b$.

Theorem 1.2. There is a primitive recursive function $t_{0}$ of two variables sucb that for all $a, b$ in $\mathcal{O}, a+_{0} b \in \mathcal{O}, 1<<_{0} b$ implies $a<_{0} a+_{0} b$, and $\left|a++_{0} b\right|=$ $|a|+|b|$.

The next theorem is proven in $[8, \mathrm{p} .339]$, by Y. Moschovakis and allows one to "subtract" $a$ from $b$ if $a \leq_{0} b$.

Theorem 1.3. There exists a primitive recursive function $\delta(a, b)$ sucb that if $a \leq_{0} b$, then $\delta(a, b) \in \mathcal{O}$ and $|a|+|\delta(a, b)|=|b|$. If $a \leq_{0} x<_{0} b$, then $\delta(a, x)<_{0} \delta(a, b)$.

Our concern in this paper is to study the hyperarithmetic predicates of the form $H_{a}(x)$ with $a \in \mathcal{O}$ under many-one reducibility. By a well-known result of Spector [13], $H_{a}(x)$ and $H_{b}(x)$ with $a, b$ in $\Theta$ have the same Turing degree iff $|a|=$ $|b|$. Earlier, Davis [1] had proven that $H_{a}(x)$ and $H_{b}(x)$ are recursively isomorphic if $|a|=|b|<\omega^{2}$. Y. Moschovakis in [8] proved that even though $H_{a}(x)$ and $H_{b}(x)$ are Turing equivalent, they need not be recursively isomorphic and, moreover, since $\leq_{m}$ and $\leq_{1}$ are the same relation on $H_{a}(x)$ [8] that Spector's result is the best possible under these notions of reducibility.

Moschovakis' results in [8] prove most useful for our more extensive study of the many-one reducibilities between $H_{a}(x)$ for $|a|=\gamma$ and we state his fundamental definition and theorem.

Definition 1.1. We say that $a^{\prime}$ is recursively majorized by $b^{\prime}$ and write $a^{\prime} \lesssim b^{\prime}$ if $a^{\prime}=3 \cdot 5^{a} \in \mathcal{O}, b^{\prime}=3 \cdot 5^{b} \in \mathcal{O},\left|a^{\prime}\right|=\left|b^{\prime}\right|$, and there is a recursive function $f(x)$ such that, for all $n,\left|a_{n}\right|<\left|b_{f(n)}\right|$.

Theorem 1.4. If $a^{\prime}=3 \cdot 5^{a} \in \mathcal{O}, b^{\prime}=3 \cdot 5^{b} \in \mathcal{O}$, and $\left|a^{\prime}\right|=\left|b^{\prime}\right|$, then $H_{a^{\prime}}(x) \leq_{m} H_{b^{\prime}}(x)$ iff $a^{\prime} \lesssim b^{\prime}$. 
This paper deals entirely with the questions about $a^{\prime} \precsim b^{\prime}$ and via Theorem 1.4 indirectly with questions about many-one degrees of $H_{a^{\prime}}(x)$ and $H_{b^{\prime}}(x)$. The following generalization of Davis' result is in [8].

Theorem 1.5. If $\gamma$ is of the form $a+1$ or $\alpha+\omega$ for some $a$, then $H_{a}(x)$ and $H_{b}(x)$ are recursively isomorphic if $|a|=|b|=\gamma$.

Now let $\mathscr{L}(\gamma)$ be the set of many-one degrees of $H_{a}(x)$ with $|a|=\gamma$ partially ordered by $\leq_{m}$. Moschovakis, in [8], proves the following results.

Theorem 1.6. $\mathscr{L}(\gamma)$ and $\mathfrak{Q}(\alpha+\gamma)$ are isomorphic as partially ordered sets for $a<\omega_{1}, \gamma<\omega_{1}\left(\omega_{1}\right.$ the first nonconstructive ordinal).

Theorem 1.7. If $\omega<\gamma<\omega_{1}$ and $\gamma$ is principal for addition, then $\mathcal{L}(\gamma)$ contains chains of order type $\omega_{1}$ and incomparable elements under $\leq_{m}$, where $\omega_{1}$ is the first nonconstructive ordinal.

It follows from these results that if $\mathcal{L}(\gamma)$ contains more than a single element, then $\gamma=\alpha+\beta$ for some $\beta \geq \omega^{2}, \beta$ a principal number for addition, and $\mathscr{L}(\gamma)$ and $\mathscr{L}(\beta)$ are isomorphic. Thus, in order to study the structure of $\mathscr{L}(\gamma)$ for all $\gamma$, it suffices to study $\mathcal{L}(\beta)$ for $\beta \geq \omega^{2}, \beta$ principal for addition.

Before we proceed to our main results, we prove the following result which is implicit in [8].

Lemma 1.1. There exists a primitive recursive function $\operatorname{sum}_{0}(e)$ sucb that if $e$ is a Gödel number of a recursive function such that for each $x,\{e\}(x) \in \mathcal{O}$ and $1<{ }_{0}\{e\}(x)$, then $\operatorname{sum}_{0}(e)=3 \cdot 5^{c} \in \mathcal{O}, c_{0}=\{e\}(0), c_{n+1}=c_{n}+_{0}\{e\}(n+1)$, and $\left|c_{n}\right|=\Sigma_{i=0}^{n}|\{e\}(i)|$.

Proof. Define $c$ to be a Gödel number of the function $g(x)$ defined inductively by

$$
\begin{aligned}
g(1) & \simeq\{e\}(0), \\
g\left((n+1)_{0}\right) & \simeq g\left(n_{0}\right)+{ }_{0}\{e\}(n+1), \\
g(x) & =1 \text { if } x \neq n_{0} \text { for some } n .
\end{aligned}
$$

The conclusions follow easily by Theorem 1.2.

Definition 1.2. If $e$ is as above, then we call $\operatorname{sum}_{0}(e)$ the infinite sum in $\theta$ of the sequence $\{e\}(0),\{e\}(1), \cdots,\{e\}(n), \cdots$ and often write $\operatorname{sum}_{0}(e)=$ $\Sigma_{0 i=0}^{\infty}\{e\}(i)$. 


\section{CHAPTER II. UNIVERSAL $\mathscr{~}(\gamma)$}

In this chapter we prove a main result of this paper, namely

Theorem 2.1. If $\gamma$ is any principal number for addition with $\omega^{2} \leq \gamma<\omega_{1}$, then $\mathcal{S}(\gamma)$ is universal as a partially ordered set, i.e., any countable partially ordered set $\mathcal{S}$ can be embedded in $\mathcal{L}(\gamma)$.

Once this is established, the following is immediate using Theorem 1.6.

Corollary 2.1. If $\gamma$ is any ordinal $0<\gamma<\omega_{1}$ not of the form $a+1$ or $a+\omega$ for any $a$, then $\mathcal{L}(\gamma)$ is universal.

The key result used in constructing $b \in \mathcal{O}$ rich in $\prec$ properties is the following

Theorem 2.2. There exists a primitive recursive function $F(r, a)$ sucb that whenever $r$ is a Gödel number of a recursive function representing a predicate of the form $(s)(s \leq t \rightarrow R(s))$ and $a \in \mathcal{O}$, then

(i) $F(r, a) \in \mathcal{O}$,

(ii) $|F(r, a)|=|a|$ if $(t)(\{r\}(t)=0)$,

(iii) $|F(r, a)| \leq \omega \cdot k_{a}$ for some $k_{a}$ if $\left(E_{t}\right)(\{r\}(t) \neq 0)$,

(iv) $|F(r, a)| \leq|a|$, and

(v) $|F(r, a)| \geq \omega$ if $|a| \geq \omega$,

(vi) moreover, if (iii) applies, then from $t$ such that $\{r\}(t) \neq 0$ we can effectively find the Gödel number of a partial recursive function $q$ such that, for all $a \in \mathcal{O}, q(a)$ converges and $|F(r, a)| \leq \omega \cdot q(a)$.

Proof. Apply Kleene's recursion theorem [4, p. 352] to the primitive recursive function $f(z, r, a)$ defined as follows:

(0) $f(z, r, a)=0$ if $a \neq 1, a \neq 2^{(a)_{0}}$, or $a \neq 3 \cdot 5^{(a)_{2}}$,

(1) $f(z, r, 1)=1$,

(2) $f\left(z, r, 2^{a}\right)=f(z, r, a)+{ }_{0} 2$, and

(3) $f\left(z, r, 3 \cdot 5^{a}\right)=3 \cdot 5^{g(z, r, a)}$ where $g(z, r, a)$ is a Gödel number of the following "system of equations":

(a)

$$
\begin{aligned}
& \{g(z, r, a)\}\left(0_{0}\right) \simeq\{z\}\left(r, a_{0}\right) \text { if }\{r\}(0)=0 \\
& \{g(z, r, a)\}\left(0_{0}\right) \simeq 2 \text { if }\{r\}(0) \neq 0, \text { undefined otherwise. }
\end{aligned}
$$




$$
\{g(z, r, a)\}\left((j+1)_{0}\right) \simeq\left(\{g(z, r, a)\}\left(j_{0}\right)+_{0}\{z\}\left(r, \delta\left(a_{j}, a_{j+1}\right)\right)\right)
$$

(b)

$$
\begin{aligned}
\{g(z, r, a)\}\left((j+1)_{0}\right) \simeq & \{g(z, r, a)\}\left(j_{0}\right)++_{0} 2, \\
& \text { if }\{r\}(j+1) \neq 0, \text { undefined otherwise. }
\end{aligned}
$$

Now by the recursion theorem we can find an $e$ such that, for all $r$ and $a$, $\{e\}(r, a) \cong f(e, r, a)$. Let $F(r, a)=\{e\}(r, a)=f(e, r, a)$, and since $f(e, r, a)$ is primitive recursive so is $F(r, a)$.

We show (i)-(v) hold for all $a \in \mathcal{O}$ by ordinal induction on $|a|$.

(i) If $|a|<\omega$, then $F(r, a)=a \in \mathcal{O}$. If $a=2^{(a)} 0$, then $\left.F(r, a)=2^{F(r,(a)}\right)_{0} \in \mathcal{O}$ since $F\left(r,(a)_{0}\right) \in \mathcal{Q}$. If $a^{\prime}=3 \cdot 5^{a}$, then $F\left(r, \delta\left(a_{j}, a_{j+1}\right)\right) \in \mathcal{O}$ since $\left|\delta\left(a_{j}, a_{j+1}\right)\right|<\left|a^{\prime}\right|$, and it is easy to show $\{g(e, r, a)\}\left(j_{0}\right)<_{0}\{g(e, r, a)\}\left((j+1)_{0}\right)$ and hence $3 \cdot 5^{g(e, r, a)}=F\left(r, a^{\prime}\right) \in \mathcal{O}$.

(ii) Suppose for all $t,\{r\}(t)=0$. Clearly, $|F(r, a)|=|a|$ if $|a|<\omega .\left|F\left(r, 2^{a}\right)\right|=$ $|F(r, a)|+1=|a|+1=\left|2^{a}\right|$, since $|a|<\left|2^{a}\right|$. Suppose $a^{\prime}=3 \cdot 5^{a}$; since $\left|a_{0}\right|<\left|a^{\prime}\right|,\left|\delta\left(a_{j}, a_{j+1}\right)\right|<a^{\prime}$ we have $\left|F\left(r, a_{0}\right)\right|=\left|a_{0}\right|,\left|F\left(r, \delta\left(a_{j}, a_{j+1}\right)\right)\right|=$ $\left|\delta\left(a_{j}, a_{j+1}\right)\right|$. Consequently, one shows easily

$$
\left|\{g(e, r, a)\}\left((j+1)_{0}\right)\right|=\sum_{i=0}^{j+1}\left|\delta\left(a_{i-1}, a_{i}\right)\right|=\left|a_{j+1}\right|,
$$

since $\left|a+{ }_{0} \delta(a, b)\right|=|a|+|\delta(a, b)|=|b|$ if $a<_{0} b$. Thus,

$$
\left|F\left(r, a^{\prime}\right)\right|=\lim _{j \rightarrow \infty}|\{g(e, r, a)\}(j)|=\lim _{j \rightarrow \infty}\left|a_{j}\right|=\left|a^{\prime}\right| .
$$

(iii) Suppose $t_{0}$ is the smallest $t$ such that $\{r\}(t) \neq 0$; then $\{r\}(t) \neq 0$ for all

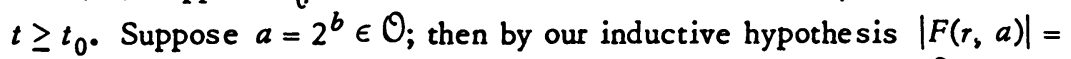
$|F(r, b)|+1 \leq \omega \cdot k_{b}+1<\omega \cdot\left(k_{b}+1\right)$. Suppose $a^{\prime}=3 \cdot 5^{a} \in \mathcal{O}$; then for each $j$, $\left|F\left(r, \delta\left(a_{j}, a_{j+1}\right)\right)\right| \leq \omega \cdot k_{j}$ for some $k_{j}$ and it follows readily that

$$
\left|F\left(r, a^{\prime}\right)\right|=\lim _{n}\left|\{g(e, r, a)\}\left(t_{0}+n\right)_{0}\right| \leq \omega \cdot \sum_{i=0}^{t_{0}-1} k_{i}+\omega .
$$

(iv) By (ii) above, we need only consider the case when $\{r\}(t) \neq 0$ for all $t \geq t_{0}$. Suppose $a^{\prime}=3 \cdot 5^{a}$, then

$$
\begin{aligned}
\left|\{g(e, r, a)\}\left(t_{0}+j\right)_{0}\right| & \leq\left|F\left(r, a_{0}\right)\right|+\sum_{i=1}^{t_{0}-1}\left|F\left(r, \delta\left(a_{i-1}, a_{i}\right)\right)\right|+(j+1) \\
& \leq\left|a_{t_{0}-1}\right|+j+1
\end{aligned}
$$


by our inductive hypothesis. Thus, $\left|F\left(r, a^{\prime}\right)\right| \leq\left|a^{\prime}\right|$.

$(v)$ is trivial.

Suppose $t_{0}$ equals the least $t$ such that $\{r\}(t) \neq 0$. We define $q$ using the recursion theorem on the partial recursive function $f(z, a)$ defined as follows:

(0) $q \quad f(z, a)=0$ if $a \neq 1, a \neq 2^{(a)_{0}}$, or $a \neq 3 \cdot 5^{(a)_{2}}$,

(1) $q \quad f(z, 1)=1$,

(2) $q$

$f\left(z, 2^{a}\right) \simeq f(z, a)+1$ if $a=3 \cdot 5^{(a)_{2}}$

$f\left(z, 2^{a}\right) \simeq f(z, a)$ if $a \neq 3 \cdot 5^{(a)_{2}}$,

$f\left(z, 3 \cdot 5^{a}\right) \simeq\left[\left(\sum_{i \in S}\{z\}\left(\delta\left(a_{i-1}, a_{i}\right)\right)\right)+\{z\}\left(a_{0}\right)\right]+1$, where $a_{0} \neq n_{0}$ for any $n$ (3) $q$ and $S=\left\{i: i \leq t_{0}-1\right.$ and $\delta\left(a_{i-1}, a_{i}\right) \neq n_{0}$ for any $\left.n\right\}$, or

$$
f\left(z, 3 \cdot 5^{a}\right) \simeq\left[\sum_{i \in S}\{z\}\left(\delta\left(a_{i-1}, a_{i}\right)\right)\right]+1 \text { if } a_{0}=n_{0}
$$

for some $n$ and $S$ is as above, undefined otherwise.

By the recursion theorem [4, p. 352] we can effectively find $e$ such that $\{e\}(a) \cong f(e, a)$. Define $q(a) \cong\{e\}(a)$. It is straightforward to show $|F(r, a)| \leq$ $w \cdot q(a)$ by induction on $|a|$ for $a \in \mathcal{O}$. Q.E.D.

We assume now that $b^{\prime}=3 \cdot 5^{b} \in \mathcal{O}, \omega^{3} \leq\left|b^{\prime}\right|$, and $\left|b^{\prime}\right|$ is a principal number for addition. We are interested in constructing elements $c^{\prime} \in \mathcal{O}$ such that $b^{\prime} \prec c^{\prime}$, so that we may assume $\left|\delta\left(b_{i-1}, b_{i}\right)\right| \geq \omega^{2}$ for each $i \geq 0$, where $b_{-1}=1$; otherwise replace $b^{\prime}$ by $d^{\prime}=\operatorname{sum}_{0}(e)=\Sigma_{\mathcal{O}_{i}=0}^{\infty}\{e\}(i)$ where $\{e\}(i)=a+_{0} \delta\left(b_{i-1}, b_{i}\right)$ where $a \in \mathcal{O}$ and $|a|=\omega^{2}$. Clearly, $b^{\prime} \lesssim d^{\prime},\left|d^{\prime}\right|=\left|b^{\prime}\right|$ and $\left|\delta\left(d_{i-1}, d_{i}\right)\right| \geqq \omega^{2}$.

We now define inductively a recursive function $f(n, t)$ (depending upon $b^{\prime}$ and a Gödel number $e$ of a partial recursive function) such that for each $n$ and $t$, $f(n, t) \in \mathcal{O}$ and $1<_{0} f(n, t)$. Moreover, we will define $\gamma_{i}=\Sigma_{O_{t}=0}^{\infty} f(i, t)$ and $c^{\prime}=$ $3 \cdot 5^{c}=\Sigma_{O i=0}^{\infty} \gamma_{i}$. The construction will require that the "growth" of $\{e\}$ determine the relative size of $c_{i}$ with respect to $b_{i}$, i.e., for each $i$ we require for $k_{i}=$ $\max \{i,\{e\}(0), \cdots,\{e\}(i)\}$ that $\left|b_{i}\right| \leq\left|b_{k_{i}}\right| \leq\left|c_{i}\right| \leq\left|b_{k_{i}}\right|+\omega^{2} \cdot(i+1)$ for each $i$. This is accomplished as follows, letting $a$ be a fixed notation for $\omega$ :

$$
\begin{aligned}
f(0,0) & =\delta\left(1, b_{0}\right), \\
\ldots & \\
f(0, t) & =\delta\left(b_{0}, b_{\{e\}(0)}\right) \text { if } T_{1}(e, 0, t) \text { and } U(t)>0, \\
f(0, t) & =a \text { otherwise, }
\end{aligned}
$$


Below $F(r, a)$ denotes the primitive recursive function of Theorem 2.2. For $n \geq 1$, define $f(n, t)$ as follows:

$f(n, 0)=F\left(r_{n}, \delta\left(b_{n-1}, b_{n}\right)\right)$, where $\left\{r_{n}\right\}(t)=0$ iff

$$
(z)\left(t^{\prime}\right)\left(\left[z<n \wedge t^{\prime} \leq t \wedge T_{1}\left(e, z, t^{\prime}\right)\right] \rightarrow\left(U\left(t^{\prime}\right)<n\right)\right) .
$$

$f(n, t)=a$ if $\bar{T}_{1}(e, n, t) \vee\left(T_{1}(e, n, t) \wedge U(t) \leq n\right)$.

$f(n, t)=F\left(r_{n}^{1}, \delta\left(b_{k_{1}}, b_{\{e\}(n)}\right)\right)$ if $T_{1}(e, n, t) \wedge U(t)>n \wedge k_{1}<U(t)$, where $k_{1}=\max \left(\left\{U\left(t^{\prime}\right): t^{\prime} \leq t \wedge(E z<n) T_{1}\left(e, z, t^{\prime}\right)\right\} \cup\{n\}\right)$ and $\left\{r_{n}^{1}\right\}(x)=0$ iff $(z)\left(t^{\prime}\right)\left(\left[t^{\prime} \leq x \wedge z<n \wedge T_{1}\left(e, z, t^{\prime}\right)\right] \rightarrow\left(U\left(t^{\prime}\right) \leq k_{1}\right)\right)$.

$f(n, t)=a$ otherwise.

After $\left.f(n, z)=F\left(r_{n}^{j}, \delta\left(b_{k_{j}}, b_{\{e}\right\}(n)\right)\right)$ for some $z$ and $j>0$, we proceed as follows for $t>z$ (otherwise continue as above).

$$
f(n, t)=a \text { if }\left\{r_{n}^{j}\right\}(t)=0 \text {. }
$$

$f(n, t)=F\left(r_{n}^{j+1}, \delta\left(b_{k_{j+1}}, b_{\{e\}(n)}\right)\right)$ if $t$ is the first $t$ such that $\left\{r_{n}^{j}\right\}(t) \neq 0$ and $k_{j+1}<\{e\}(n)$, where $k_{j+1}=\max \left\{U\left(t^{\prime}\right): t^{\prime} \leq t \wedge(E m<n) T_{1}(e, m, t) \wedge U\left(t^{\prime}\right)>k_{j}\right\}$ and $\left\{r_{n}^{j+1}\right\}(x)=0$ iff $(z)\left(t^{\prime}\right)\left(\left[t^{\prime} \leq x \wedge z<n \wedge T_{1}\left(e, z, t^{\prime}\right)\right] \rightarrow\left(U\left(t^{\prime}\right) \leq k_{j+1}\right)\right)$.

$f(n, t)=a$ otherwise.

For each $i$, let $\gamma_{i}=\Sigma_{\theta_{t=0}}^{\infty} f(i, t)$, via Lemma 1.1. For $i=0$, it is clear that $\left|\gamma_{0}\right|=\left|\delta\left(1, b_{0}\right)\right|+\omega \cdot q+\left|\delta\left(b_{0}, b_{\{e\}(0)}\right)\right|+\omega^{2}$ if $\{e\}(0)$ is defined and $\{e\}(0)>0$ or $\left|\gamma_{0}\right|=\left|\delta\left(1, b_{0}\right)\right|+\omega^{2}$ if either $\{e\}(0)$ is undefined or $\{e\}(0)=0$. The following lemma clarifies how the function $\{e\}(n)$ determines the size of $\left|\gamma_{i}\right|$.

Lemma 2.1. For $i>0$, let $n_{i-1}=\max \{i-1,\{e\}(0), \cdots,\{e\}(i-1)\}$; then for some $q$

$$
\begin{aligned}
& \left.\left|\gamma_{i}\right|=\left|\delta\left(b_{i-1}, b_{i}\right)\right|+\omega \cdot q+\mid \delta\left(b_{i}, b_{\{e}\right\}_{(i)}\right) \mid+\omega^{2} \text { if } n_{i-1}<i \\
& \text { and } i<\{e\}(i) \text {, } \\
& \left|\gamma_{i}\right|=\left|\delta\left(b_{i-1}, b_{i}\right)\right|+\omega^{2} \text { if } n_{i-1}<i \text { and either } \\
& \{e\}(i) \text { is undefined or }\{e\}(i) \leq i \text {, } \\
& \begin{aligned}
\left.\left|\gamma_{i}\right|=\omega \cdot q+\mid \delta\left(b_{n_{i-1}}, b_{\{e}\right\}_{(i)}\right) \mid+\omega^{2} & \text { if } i \leq n_{i-1} \text { and } \\
n_{i-1} & <\{e\}(i), \text { or }
\end{aligned} \\
& \left|\gamma_{i}\right|=\omega^{2} \quad \text { if } i \leq n_{i-1} \text { and either } \\
& \{e\}(i) \text { is undefined or }\{e\}(i) \leq n_{i-1} \text {. }
\end{aligned}
$$


Proof. Suppose $i>n_{i-1}$, then $n_{i-1}=i-1$. Moreover, $|f(i, 0)|=$ $\left|\delta\left(b_{i-1}, b_{i}\right)\right|$ by Theorem 2.2 , since $\left\{r_{i}\right\}(t)=0$ holds for all $t$. If $\{e\}(i)$ is undefined, then $f(i, t)=a$ for all $t>0$ and thus $(2)_{i}$ holds. If $\{e\}(i)$ is defined, then for some unique $t^{\prime}, T_{1}\left(e, i, t^{\prime}\right)$ and $U\left(t^{\prime}\right)=\{e\}(i)$. If $\{e\}(i) \leq i$, then $f(i, t)=a$ for all $t>0$ and again $(2)_{i}$ holds. If $\{e\}(i)>i$, then $U\left(t^{\prime}\right)>i=k_{1} ;\left\{r_{i}^{1}\right\}(x)=0$ for all $x$, and thus $\left.\left|f\left(i, t^{\prime}\right)\right|=\mid \delta\left(b_{i}, b_{\{e}\right\}(i)\right) \mid$ from which $(1)_{i}$ follows since $f(i, t)=a$ for $t \neq 0$ and $t \neq t^{\prime}$.

Suppose $i \leq n_{i-1}$; then $\left\{r_{i}\right\}(t) \neq 0$ for some $t$ since there is a $z<i$ such that $\{e\}(z)=n_{i-1} \geq i$. Hence, by Theorem $2.2,|f(i, 0)| \leq \omega \cdot q_{1}$ for some $q_{1}$. Suppose $\{e\}(i)$ is not defined; then $f(i, t)=a$ for $t>0$ and consequently $(4)_{i}$ holds. Suppose $\{e\}(i) \leq n_{i-1}$; then let $t^{\prime}$ be the smallest $t$ such that, for some $z<i$, $T_{1}(e, z, t) \wedge U(t) \geq\{e\}(i)$. Let $t_{i}$ be the unique $t$ such that $T_{1}(e, i, t)$. If $\{e\}(i) \leq i$, then $f(i, t)=a$ for all $t>0$ since $\{e\}(i) \leq i \leq k_{1}$; thus (4) holds. Suppose now $i<\{e\}(i) \leq n_{i-1}$. If $t^{\prime} \leq t_{i}$, then $\{e\}(i) \leq k_{1}, f(i, t)=a$ for all $t>0$, and $(4)_{i}$ holds. If $t_{i}<t^{\prime}$, then $k_{1}<\{e\}(i),\left\{r_{i}^{\prime}\right\}\left(t^{\prime}\right) \neq 0$, and $\left|f\left(i, t_{i}\right)\right| \leq \omega \cdot q_{1}$ for some $q_{1}$ by Theorem 2.2. For any $r_{i}^{j}$ defined before $f\left(i, t^{\prime}\right)$ is defined, it is clear that $\left\{r_{i}^{j}\right\}\left(t^{\prime}\right) \neq 0$ and hence $|f(i, t)| \leq \omega \cdot q^{\prime}$ for some $q^{\prime}$ and each $t<t^{\prime}$ by Theorem 2.2. However, $f\left(i, t^{\prime}\right)=a$ since $\{e\}(i) \leq U\left(t^{\prime}\right) \leq n_{i-1}$ and thus it follows that $f(i, t)=a$ for all $t \geq t^{\prime}$ and (4) $i$ holds.

Suppose $i \leq n_{i-1}$ and $n_{i-1}<\{e\}(i)$. As above, it follows that $|f(i, 0)| \leq$ $\omega \cdot q_{1}$ for some $q_{1}$. Let $t^{\prime}$ now be the smallest $t$ such that, for some $z<i$, $T_{1}\left(e, z, t^{\prime}\right)$ and $U\left(t^{\prime}\right)=n_{i-1}$. Let $t_{i}$ be that unique $t$ such that $T_{1}(e, i, t)$. If $t^{\prime} \leq t_{i}$, then $k_{1}=n_{i-1},\left\{r_{i}^{1}\right\}(x)=0$ for all $x$, and $\left|f\left(i, t_{i}\right)\right|=\left|\delta\left(b_{n_{i-1}}, b_{\{e}\right\}(i)\right|$ by Theorem 2.2; hence (3) holds. If $t_{i}<t^{\prime}$ then, for each $0<t<t^{\prime}, f(i, t)=a$ or $\left.f(i, t)=\Gamma\left(r_{i}^{j}, \delta\left(b_{k_{j}}, b_{\{e}\right\}(i)\right)\right)$ where $k_{j}<n_{i-1}$ and consequently $\left\{r_{i}^{j}\right\}\left(t^{\prime}\right) \neq 0$. Hence, $|f(i, t)| \leq \omega \cdot q$ for some $q$ for all $t<t^{\prime}$, by Theorem 2.2. If $j^{\prime}$ is the largest $j$ such that $\left.f(i, t)=F\left(r_{i}^{j}, \delta\left(b_{k_{j}}, b_{\{e}\right\}(i)\right)\right)$ for some $t<t^{\prime}$, then $\left\{r_{i}^{j^{\prime}}\right\}\left(t^{\prime}\right) \neq 0$ since $k_{j}<n_{i-1}=U\left(t^{\prime}\right)\left(t^{\prime}\right.$ is the smallest $t$ such that $\left.\left\{r_{i}^{j^{\prime}}\right\}(t) \neq 0\right), k_{j^{\prime}+1}=n_{i-1}$, $k_{j^{\prime}+1}<\{e\}(i)$, and $f\left(i, t^{\prime}\right)=F\left(r_{i}^{j^{\prime}+1}, \delta\left(b_{n_{i-1}}, b_{\{e\}(i)}\right)\right)$ where $\left\{r_{i}^{j^{\prime}+1}\right\}(t)=0$ for all $t$. Hence, $f(i, t)=a$ for all $t>t^{\prime}$. Consequently, $\left|f\left(i, t^{\prime}\right)\right|=$ $\left|\delta\left(b_{n_{i-1}}, b_{\{e\}(i)}\right)\right| ;$ hence $(3)_{i}$ holds.

Lemma 2.2. There exists a primitive recursive function $G\left(e, b^{\prime}\right)$ such that if $b^{\prime}=3 \cdot 5^{b} \in \mathcal{C}$ and $b^{\prime}$ is as assumed above $\left(\left|b^{\prime}\right|>\omega^{2}\right.$, for eacb $i$, $\left|\delta\left(b_{i-1}, b_{i}\right)\right| \geq \omega^{2},\left|b^{\prime}\right|$ principal for addition $)$, then $G\left(e, b^{\prime}\right)=c^{\prime}=3 \cdot 5^{c} \in \mathcal{O}$ and $\left|c^{\prime}\right|=\left|b^{\prime}\right|$. Moreover, for eacb $n$,

$$
\left|b_{n}\right| \leq\left|b_{k_{n}}\right| \leq\left|c_{n}\right| \leq\left|b_{k_{n}}\right|+\omega^{2} \cdot(n+1),
$$

where $k_{n}=\max \{n,\{e\}(0), \cdots,\{e\}(n)\}$. Clearly, then $b^{\prime} \lesssim c^{\prime}$. 
Proof. The existence of $G\left(e, b^{\prime}\right)$ is clear by our construction of $f(n, t)$, the remarks preceding that construction, and Lemma 1.1. By Lemma 1.1,

$$
\left|c_{n}\right|=\sum_{O i=0}^{\infty}\left|\gamma_{i}\right| \text { where } \gamma_{i}=\sum_{O t=0}^{\infty} f(i, t) .
$$

Clearly, $k_{0} \leq k_{1} \leq \cdots \leq k_{n}$ where $k_{i}=\max \{i,\{e\}(0), \cdots,\{e\}(i)\}$. Define $i_{0}=0$, $i_{j+1}=m$, where $m$ is the smallest number such that $k_{m}>k_{i_{j}}$. Suppose $k_{i_{q}}=k_{n}$, $q \geq 0$ and $i_{0}<i_{1}<\cdots<i_{q}$. Since $\left|\delta\left(b_{i-1}, b_{i}\right)\right| \geq \omega^{2}$, then for any $q, \omega \cdot q+$ $\left|\delta\left(b_{i}, b_{j}\right)\right|=\left|\delta\left(b_{i}, b_{j}\right)\right|$ if $i<j$. By Lemma 2.1 , it follows easily that

$$
\begin{aligned}
\left|c_{n}\right|=\sum_{i=0}^{n}\left|\gamma_{i}\right|=\mid & \left|\delta\left(1, b_{k_{i_{0}}}\right)\right|+\omega^{2}+\omega^{2} \cdot\left(i_{1}-\left(i_{0}+1\right)\right) \\
& +\left|\delta\left(b_{k_{i_{0}}}, b_{k_{i_{1}}}\right)\right|+\omega^{2}+\omega^{2} \cdot\left(i_{2}-\left(i_{1}+1\right)\right)+\cdots \\
& +\left|\delta\left(b_{k_{i_{q-1}}}, b_{k_{i_{q}}}\right)\right|+\omega^{2}+\omega^{2} \cdot\left(n-i_{q}\right) .
\end{aligned}
$$

It is clear since $k_{i_{q}}=k_{n}$ that $\left|b_{n}\right| \leq\left|b_{k_{n}}\right| \leq\left|c_{n}\right|$. If $\alpha$ is any ordinal such that $a \geq \omega^{2}$, then $\omega^{2}+\alpha^{q}+\omega^{2} \leq \alpha+\omega^{2}+\omega^{2}$ by the Cantor normal form of $\alpha$ [0]; consequently, $\left|c_{n}\right| \leq\left|b_{k_{n}}\right|+\omega^{2} \cdot(n+1)$. Thus, (1) holds.

Definition 2.1. We say that a partial recursive function $\{e\}(x)$ has a recursive upper bound if there exists a recursive function $f(x)$ such that $\{e\}(i) \leq f(i)$ for every $i$ in the domain of $\{e\}$. We call $f(x)$ a recursive upper bound for $\{e\}(x)$.

Lemma 2.3. Let e be the Gödel number of a partial recursive function and $G\left(e, b^{\prime}\right)=c^{\prime}=3 \cdot 5^{c}$ be given by Lemma 2.2. $\{e\}(x)$ bas no recursive upper bound iff $b^{\prime}<c^{\prime}$.

Proof. Clearly, by Lemma $2.2, b^{\prime} \precsim c^{\prime}$. Suppose $c^{\prime} \lesssim b^{\prime}$. Then there is a recursive function $g(x)$ such that for all $n,\left|c_{n}\right|<\left|b_{g(n)}\right|$. Let $k_{n}=$ $\max \{n,\{e\}(0), \cdots,\{e\}(n)\}$. By Lemma 2.2, $\left|b_{k_{n}}\right| \leq\left|c_{n}\right|<\left|b_{g(n)}\right|$. Hence, $k_{n}<g(n)$ and clearly $g(x)$ is a recursive upper bound of $\{e\}(x)$.

Suppose $\{e\}(x)$ has a recursive upper bound $f(x)$. Define $b(x)=x+$ $\left(\sum_{i=0}^{x} f(x)\right)+x+1$. By Lemma 2.2, $\left|c_{n}\right| \leq\left|b_{k_{n}}\right|+\omega^{2} \cdot(n+1) \leq\left|b_{k_{n}}+(n+1)\right| \leq$ $\left|b_{b(n)}\right|$. Hence $c^{\prime} \precsim b^{\prime}$.

The following lemma of S. Tennenbaum shows that there exists a vast number of partial recursive functions $\{e\}(x)$ without recursive upper bounds and gives an alternative characterization of the notion of a nonrecursive recursively enumerable set. 
Lemma 2.4. Let $W_{e}$ be any infinite r.e. set and let $f(x)$ be a recursive func. tion mapping $N$ one-one onto $W_{e}$. Then $f^{-1}(x)$ is a partial recursive function without a recursive upper bound iff $W_{e}$ is nonrecursive.

Proof. Suppose $W_{e}$ is recursive, then define

$$
\begin{array}{ll}
b(x)=f^{-1}(x), & \text { if } x \in W_{e}, \\
b(x)=0, & \text { if } x \notin W_{e} .
\end{array}
$$

Since $W_{e}$ is recursive, so is $b(x)$ and clearly $b(x)$ is a recursive upper bound for $f^{-1}(x)$.

Suppose $f^{-1}(x)$ has a recursive upper bound, say $b(x)$. Then $x \in W_{e}$ iff $(E y \leq b(x))(f(y)=x)$. Since $b(x)$ and $f(x)$ are both recursive, it is clear that $W_{e}$ is recursive.

Definition 2.2. If $e_{1}$ and $e_{2}$ are Gödel numbers of partial recursive functions, then we say $\left\{e_{1}\right\}(x)$ is recursively maximized by $\left\{e_{2}\right\}(x)$ and write $\left\{e_{1}\right\} \leq^{m}$ $\left\{e_{2}\right\}$ if there is a recursive function $f(x)$ such that, for each $n$,

$$
\max \left\{n,\left\{e_{1}\right\}(0), \ldots,\left\{e_{1}\right\}(n)\right\} \leq \max \left\{f(n),\left\{e_{2}\right\}(0), \cdots,\left\{e_{2}\right\}(f(n))\right\} .
$$

Lemma 2.5. If $\left\{e_{1}\right\} \leq^{m}\left\{e_{2}\right\}$, then $G\left(e_{1}, b^{\prime}\right) \lesssim G\left(e_{2}, b^{\prime}\right)$.

Proof. Suppose $c^{\prime}=3 \cdot 5^{c}=G\left(e_{1}, b^{\prime}\right)$ and $d^{\prime}=3 \cdot 5^{d}=G\left(e_{2}, b^{\prime}\right)$. Suppose that, for some recursive function $f(x)$,

$$
\begin{aligned}
k_{n} & =\max \left\{0,\left\{e_{1}\right\}(0), \ldots,\left\{e_{1}\right\}(n)\right\} \\
& \leq k_{f(n)}^{\prime}=\max \left\{f(n),\left\{e_{2}\right\}(0), \ldots,\left\{e_{2}\right\}(f(n))\right\} .
\end{aligned}
$$

By Lemma 2.2, we have $\left|c_{n}\right| \leq\left|b_{k_{n}}\right|+\omega^{2} \cdot(n+1)$ and $\left|b_{k_{n}}\right| \leq\left|b_{k_{f(n)}^{\prime}}\right| \leq\left|d_{f(n)}\right| \cdot$ Consequently, $\left|c_{n}\right| \leq\left|d_{f(n)}\right|+\omega^{2} \cdot(n+1)$. By Lemma 2.1 as applied in the proof of Lemma 2.2, $\left|\delta\left(d_{i}, d_{i+1}\right)\right| \geq \omega^{2}$ and, hence, $\left|c_{n}\right| \leq\left|d_{f(n)_{+}(n+1)}\right|$. Define $g(x)=$ $f(x)+x+1$. Then, for every $n,\left|c_{n}\right| \leq\left|d_{g(n)}\right|$ and, by definition, we have

$$
c^{\prime}=G\left(e_{1}, b^{\prime}\right) \lesssim d^{\prime}=G\left(e_{2}, b^{\prime}\right) \text {. }
$$

The next result demonstrates how we can construct two elements $c^{\prime}$ and $d^{\prime}$ incomparable under $\precsim$ such that $b^{\prime}<c^{\prime}$ and $b^{\prime}<d^{\prime}$ once we know the existence of two r.e. sets with incomparable Turing degrees.

Lemma 2.6. Let $f_{1}(x)$ and $f_{2}(x)$ be one-one recursive functions mapping the recursive sets $R_{1}$ and $R_{2}$ one-one onto $W_{1}$ and $W_{2}$, respectively. Let $e_{1}$ and $e_{2}$ be Gödel numbers of $f_{1}^{-1} / W_{1}$ and $f_{2}^{-1} / W_{2}$, respectively, where $f_{i}^{-1} / W_{i}$ denotes 
the restriction of $f_{i}^{-1}(x)$ to domain $W_{i}, i=1$, 2. Then $G\left(e_{1}, b^{\prime}\right) \precsim G\left(e_{2}, b^{\prime}\right)$ implies $W_{1} \leq_{T} W_{2}$.

Proof. Let $c^{\prime}=3 \cdot 5^{c}=G\left(e_{1}, b^{\prime}\right)$ and $d^{\prime}=3 \cdot 5^{d}=G\left(e_{2}, b^{\prime}\right)$. Suppose $g(x)$ is a recursive function such that, for each $n,\left|c_{n}\right| \leq\left|d_{g(n)}\right|$. Let

$$
k_{n}=\max \left\{0,\left\{e_{1}\right\}(0), \cdots,\left\{e_{1}\right\}(n)\right\}
$$

and

$$
\left.k_{g(n)}^{\prime}=\max \left\{g(n),\left\{e_{2}\right\}(0), \ldots,\left\{e_{2}\right\} g(n)\right)\right\}
$$

By Lemma 2.2, we have for each $n$,

$$
\left|b_{k_{n}}\right| \leq\left|c_{n}\right| \leq\left|d_{g(n)}\right| \leq\left|b_{k_{g(n)}^{\prime}}\right|+\omega^{2} \cdot(g(n)+1) \leq \mid b_{k_{g(n)}^{\prime}}+(g(n)+1),
$$

where the last inequality holds since $\left|\delta\left(b_{i}, b_{i+1}\right)\right| \geq \omega^{2}$. Thus, $k_{n} \leq k_{g(n)}^{\prime}+$ $(g(n)+1)$.

We claim that $x \in W_{1}$ iff $E z \leq\left(k_{g(x)}^{\prime}+g(x)+1\right)\left(z \in R_{1}\right.$ and $\left.f_{1}(z)=x\right)$. Suppose $x \in W_{1}$, then there exists $z \in R_{1}$ such that $f_{1}(z)=x$ and hence $\left\{e_{1}\right\}(x)=$ $f_{1}^{-1}(x)=z \leq k_{x} \leq k_{g(x)}^{\prime}+g(x)+1$. Hence, $E z \leq\left(k_{g(x)}^{\prime}+g(x)+1\right)\left(z \in R_{1}\right.$ and $\left.f_{1}(z)=x\right)$ and, clearly, if this holds, then $x \in W_{1}$. However,

$$
u(x)=\left(\max \left\{g(x),\left\{e_{2}\right\}(0), \ldots,\left\{e_{2}\right\}(g(x))\right\}\right)+g(x)+1
$$

is recursive in $W_{2}$ since to compute $u(x)$ we need only to know those elements $y \leq g(x)$ such that $y \in W_{2}$. Thus, $E z \leq u(x)\left(z \in R_{1}\right.$ and $\left.f_{1}(z)=x\right)$ is recursive in $W_{2}$. Hence, $W_{1} \leq_{T} W_{2}$.

Sacks, in [12], has proven that there exists an infinite sequence $A_{0}, A_{1}, \cdots$, $A_{n}, \cdots$ of recursively enumerable sets such that, for $i \neq j, A_{i}$ and $A_{j}$ have incomparable degrees of recursive unsolvability. From this we have immediately the following result:

Corollary 2.2. There exists an infinite sequence $c_{0}^{\prime}=G\left(e_{0}, b^{\prime}\right), \cdots, c_{n}^{\prime}=$ $G\left(e_{n}, b^{\prime}\right), \cdots$ such that for $i \neq j$, then $c_{i}^{\prime}$ and $c_{j}^{\prime}$ are incomparable with respect to $\lesssim$ and $b^{\prime}<c_{i}^{\prime}$.

Proof. Let $f_{i}$ be a recursive function mapping $N$ one-one onto $A_{i}$. Let $e_{i}$ be a Gödel number of $f_{i}^{-1}(x)$. By Lemma 2.6 the result is immediate.

The following lemma allows us to relate other results about Turing degrees of r.e. sets to $\mathfrak{l}(\gamma)$.

Lemma 2.7. Suppose $f(x)$ is a one-one recursive function such that, for 
$i=1,2, f(x)$ maps the recursive set $R_{i}$ onto $A_{i}$. Suppose $A_{1} \subseteq A_{2}$ and bence $R_{1} \subseteq R_{2}$. Let $\left\{e_{i}\right\}(x)=f^{-1}(x) / A_{i}, i=1,2$. Then $G\left(e_{1}, b^{\prime}\right) \precsim G\left(e_{2}, b^{\prime}\right)$, and $A_{1}<_{T} A_{2}$ implies $G\left(e_{1}, b^{\prime}\right) \prec G\left(e_{2}, b^{\prime}\right)$.

Proof. It is clear that $A_{1} \leq_{T} A_{2}$ since $x \in A_{1}$ iff $\left(x \in A_{2} \wedge f^{-1}(x) \in R_{1}\right)$. Since $A_{1} \subseteq A_{2}$, we have

$$
\left\{i,\left\{e_{1}\right\}(0), \ldots,\left\{e_{1}\right\}(i)\right\} \subseteq\left\{i,\left\{e_{2}\right\}(0), \ldots,\left\{e_{2}\right\}(i)\right\}
$$

and, thus,

$$
\max \left\{i,\left\{e_{1}\right\}(0), \ldots,\left\{e_{1}\right\}(i)\right\} \leq \max \left\{i,\left\{e_{2}\right\}(0), \ldots,\left\{e_{2}\right\}(i)\right\}
$$

Consequently, $\left\{e_{1}\right\} \leq^{m}\left\{e_{2}\right\}$ and by Lemma 2.5 we have immediately $G\left(e_{1}, b^{\prime}\right) \precsim$ $G\left(e_{2}, b^{\prime}\right)$.

Suppose $A_{1}<_{T} A_{2}$ and suppose on the contrary that $G\left(e_{2}, b^{\prime}\right) \precsim G\left(e_{1}, b^{\prime}\right)$, i.e., not $\left(G\left(e_{1}, b^{\prime}\right) \prec G\left(e_{2}, b^{\prime}\right)\right)$. By Lemma 2.6, we have that $A_{2} \leq_{T} A_{1}$, a contradiction. Thus, $G\left(e_{1}, b^{\prime}\right)<G\left(e_{2}, b^{\prime}\right)$.

Now we prove Theorem 2.1 for $\gamma \geq \omega^{3}$.

Proof of Theorem 2.1 for $\gamma \geq \omega^{3}$. In [12, p. 53] Sacks proves the analogous result for the partially ordered set of Turing degrees of the r.e. sets by constructing an infinite sequence $B_{0}, B_{1}, \cdots, B_{n}, \cdots$ of r.e. sets having the following properties: $B_{0}, B_{1}, \cdots, B_{n}, \cdots$ is a sequence of recursively independent, disjoint, simultaneously recursively enumerable sets. Thus, there is a one-one recursive function $f(x)$ such that $f(x)$ maps $N$ one-one onto $\bigcup\left\{B_{i}: i \in N\right\}$ and $f(\{(i, n): n \in N\})=B_{i}$ for each $i$, where by $(x, y)$ we mean $t(x, y)=$ $1 / 2\left(x^{2}+2 x y+y^{2}+3 x+y\right)$ which is Cantor's pairing function that maps $N \times N$ one-one onto $N$. Sacks' proof utilizes a result due to Mostowski [9] which gives a recursive partial ordering relation $x \leq_{R} y$ on $N$ which is universal. Define $C_{u}=\bigcup\left\{B_{i}: i \leq_{R} u\right\}$. Sacks shows that

$$
u \leq_{R} v \text { iff } C_{u} \leq_{T} C_{v}
$$

We note first that $u \leq_{R} v$ implies $C_{u} \subseteq C_{v}$ since $i \leq_{R} u$ and $u \leq_{R} v$ implies $i \leq_{R} v$ by the transitivity of $\leq_{R}$. Thus, (2) is equivalent to

$$
u \leq_{R} v \text { iff } C_{u} \subseteq C_{v} \text { and } C_{u} \leq_{T} C_{v} \text {, noting that }
$$

$$
C_{u} \subseteq C_{v} \text { implies } u \leq_{R} v \text { since the } B_{i} \text { 's are disjoint }
$$
and $i \leq_{R} i$.

Now define $R_{u}=\left\{(i, n): n \geq 0 \wedge i \leq_{R} u\right\}$. It is clear that $R_{u}$ is recursive since $(x, y) \in R_{u}$ iff $x \leq_{R} u$. Also, $f(x)$ maps the recursive set $R_{u}$ one-one onto 
$C_{u}$. It is not difficult to find a primitive recursive function $p(x)$ such that for each $u, p(u)$ is a Gödel number of the partial recursive function $f^{-1}(x) / C_{u}$. Define $e_{u}=p(u)$. We claim

$$
u \leq_{R} v \quad \text { iff } G\left(e_{u}, b^{\prime}\right) \lesssim G\left(e_{v}, b^{\prime}\right),
$$

where $G\left(e, b^{\prime}\right)$ is the primitive recursive function of Lemma 2.2.

Suppose $u \leq_{R} v$, then by (3) $C_{u} \subseteq C_{v}$ and $f$ maps the recursive set $R_{u}$ oneone onto $C_{u}$. By Lemma 2.7, we have immediately $G\left(e_{u}, b^{\prime}\right) \lesssim G\left(e_{v}, b^{\prime}\right)$ and moreover $G\left(e_{u}, b^{\prime}\right)\left\langle G\left(e_{v}, b^{\prime}\right)\right.$ if $u \neq v$.

Suppose $G\left(e_{u}, b^{\prime}\right) \precsim G\left(e_{v}, b^{\prime}\right)$. Then, by Lemma 2.6, $C_{u} \leq_{T} C_{v}$ and hence $u \leq_{R} v$ by (2). Thus, (4) holds for all $u$ and $v$.

Now by Theorem 1.4, we have

$$
u \leq_{R} v \text { iff } H_{G\left(e_{u} b^{\prime}\right)}(x) \leq_{m} H_{G\left(e_{v}, b^{\prime}\right)}(x)
$$

Thus, the ordering $R$ is isomorphic to $\left\{\left[H_{G\left(e_{u}, b^{\prime}\right)}(x)\right]_{m}: u \geq 0\right\}$ under $\leq_{m}$. Since any countable partially ordered set $\delta$ can be embedded in the ordering $R$, then clearly $\mathcal{S}$ can be embedded in $\left\{\left[H_{G\left(e_{u}, b^{\prime}\right)}(x)\right]_{m}: u \geq 0\right\} \subseteq \mathcal{L}(\gamma)$ and, hence, the theorem follows for $\gamma \geq \omega^{3}$.

In order to show $\mathcal{L}\left(\omega^{2}\right)$ is universal, one follows essentially the same sequence as above but the basic construction is much easier.

Let $a$ be a fixed element of $\mathcal{O}$ such that $|a|=\omega$. We write $a \cdot k$ for $\left(\cdots\left(a+_{0} a\right)+{ }_{0} \cdots++_{0} a\right)$ with $k$ summands if $k>0$. For a Gödel number $e$ define $f(n, t)$ inductively as follows:

$$
\begin{array}{ll}
f(i, t)=2 & \text { if } \overline{T_{1}}(e, i, t), \\
f(i, t)=a \cdot k & \text { if } T_{1}(e, i, t) \text { and } U(t)=k>0, \\
f(i, t)=2, & \text { otherwise, } \\
\ldots &
\end{array}
$$

Using Lemma 1.1, define $\gamma_{i}=\Sigma_{\theta_{t=0}}^{\infty} f(i, t)$ and $c^{\prime}=3 \cdot 5^{c}=\Sigma_{\theta_{i=0}}^{\infty} \gamma_{i^{\circ}}$. It is clear that

$$
\begin{aligned}
& \left|\gamma_{i}\right|=\omega \text { if }\{e\}(i) \text { is undefined or }\{e\}(i)=0 . \\
& \left|\gamma_{i}\right|=\omega \cdot k+\omega \text { if }\{e\}(i)=k>0 .
\end{aligned}
$$

For a partial recursive function $\{e\}(x)$, we define $k_{x}=\Sigma_{i=0}^{x}\{e\}(x)=$ $\Sigma_{i \in S}\{e\}(i)$ where $S=\{i:\{e\}(i)$ is defined and $i \leq x\}$. By Lemma 1.1, we have immediately that $\left|c_{n}\right|=\omega \cdot k_{x}+\omega \cdot(n+1)$.

These facts we formulate in the following lemma. 
Lemma 2.8. There exists a primitive recursive function $G(e)$ such that, for eacb e, $G(e)=3 \cdot 5^{c}=c^{\prime} \in \mathcal{O}$ and $\left|c^{\prime}\right|=\omega^{2}$, for some $c$. Moreover, $\left|c_{n}\right|=$ $\omega \cdot k_{n}+\omega \cdot(n+1)$.

Lemma 2.9. If $b^{\prime} \in \mathcal{O},\left|b^{\prime}\right|=\omega^{2}$, and $b^{\prime}=3 \cdot 5^{b}$ is the minimum element with respect to $\lesssim, e_{.} g_{\cdot},\left|b_{n}\right|=\omega \cdot n$ for eacb $n$, then $b^{\prime}\left\langle G(e)=3 \cdot 5^{c}=c^{\prime}\right.$ iff the partial recursive function $\{c\}(x)$ bas no recursive upper bound.

Proof. Suppose $c^{\prime} \precsim b^{\prime}$, then there is a recursive function $g(x)$ such that $\left|c_{n}\right| \leq\left|b_{g(n)}\right|=|\omega| \cdot g(n)$ for each $n$. Thus, $k_{n}^{\prime}=\Sigma_{i=0}^{n}\{e\}(i)+n+1 \leq g(n)$ for each $n$ by Lemma 2.8. Clearly, then $g(n)$ is a recursive upper bound for $\{e\}(n)$.

Suppose $b^{\prime} \prec c^{\prime}$. If $g(x)$ were a recursive upper bound for $\{e\}(x)$, then $b(x)=$ $\left(\sum_{i=0}^{x} g(x)\right)+x+1$ is a recursive function. However, by Lemma 2.8 it follows that $\left|c_{n}\right| \leq\left|b_{b(n)}\right|$, a contradiction. Thus, $\{e\}(x)$ has no recursive upper bound.

Lemma 2.10. Suppose, for $i=1,2 f_{i}(x)$ is a one-one recursive function mapping $R_{i}$ onto $W_{i}$. Let $e_{i}$ be a Gödel number of $f_{i}^{-1}(x) / W_{i}$. Then $G\left(e_{1}\right) \lesssim$ $G\left(e_{2}\right)$ implies $W_{1} \leq_{T} W_{2}$.

Proof. Suppose $G\left(e_{1}\right)=c^{\prime}=3 \cdot 5^{c}$ and $G\left(e_{2}\right)=d^{\prime}=3 \cdot 5^{d}$. Let $b(x)$ be a recursive function such that $\left|c_{n}\right| \leq\left|d_{b(n)}\right|$ assuming $c^{\prime} \lesssim d^{\prime}$. Thus, by Lemma 2.8,

$$
\left(\sum_{i=0}^{n}\left\{e_{1}\right\}(i)\right)+n+1 \leq\left(\sum_{i=0}^{b(n)}\left\{e_{2}\right\}(i)\right)+b(n)+1 \text {. }
$$

Hence, $x \in W_{1}$ iff $E z \leq\left(b(x)+1+\sum_{i=0}^{b(n)}\left\{e_{2}\right\}(i)\right)\left(z \in R_{1}\right.$ and $\left.f_{1}(z)=x\right)$. Since $\Sigma_{i=0}^{b(n)}\left\{e_{2}\right\}(x)$ is recursive in $W_{2}, W_{1} \leq_{T} W_{2}$.

Lemma 2.11. Suppose $W_{1} \subseteq W_{2}$ and $f(x)$ is a one-one recursive function mapping the recursive set $R_{i}$ onto $W_{i}$ for $i=1,2$. Let $\left\{e_{i}\right\}=f^{-1}(x) / W_{i}, i=1,2$. Then $G\left(e_{1}\right) \precsim G\left(e_{2}\right)$, and $W_{1}<_{T} W_{2}$ implies $G\left(e_{1}\right) \prec G\left(e_{2}\right)$.

Proof. Clearly, by 2.8, $G\left(e_{1}\right) \precsim G\left(e_{2}\right)$ since $\Sigma_{i=0}^{n}\left\{e_{1}\right\}(i) \leq \Sigma_{i=0}^{n}\left\{e_{2}\right\}(i)$. If $G\left(e_{2}\right) \precsim G\left(e_{1}\right)$, then $W_{2} \leq_{T} W_{1}$ by 2.10 . Hence, the result follows.

Clearly now, the same proof as above for $\gamma \geq \omega^{3}$ holds, using Lemmas 2.82.11 for justification. Thus, $\mathscr{L}\left(\omega^{2}\right)$ is universal and Theorem 2.1 is established.

Theorem 2.3. If $\omega^{2} \leq y<\omega_{1}$ and $\gamma$ is not of the form $\alpha+1$ or $\alpha+\omega$ for any ordinal $a$, then $\mathcal{L}(y)$ is universal as a partially ordered set.

Proof. Immediate by Theorem 2.1 and Theorem 1.6. 


\section{CHAPTER III. NONEXISTENCE OF MINIMAL ELEMENTS}

The main result of this chapter is the following theorem:

Theorem 3.1. If $b^{\prime} \in \mathcal{O},\left|b^{\prime}\right| \geq \omega^{3}$, and $\left|b^{\prime}\right|$ is a principal number for addition, then we can effectively find $a^{\prime}, c^{\prime} \in \mathcal{O}$ sucb that $a^{\prime}<b^{\prime}, c^{\prime}<b^{\prime}$, and $a^{\prime}$, $c$ ' are incomparable under $\lesssim$.

First, we may assume without loss in generality that, for each $i, \delta\left(b_{i-1}, b_{i}\right)$ is a limit notation, for otherwise replace $b^{\prime}$ by $d^{\prime}$ with $d^{\prime} \precsim b^{\prime}$ constructed as follows: Let $g(x)$ be the recursive function defined so that $g(x)=3 \cdot 5^{y}$ if there is a notation $k$ in $\mathcal{O},|k|$ finite such that $3 \cdot 5^{y}+{ }_{0} k=x$, and $g(x)=0$ otherwise. Define $f(0)=\mu i\left(g\left(b_{i}\right) \neq 0\right)$ and $f(j+1)=\mu i\left(g\left(b_{f(j)}\right)<_{0} g\left(b_{i}\right)\right)$. Since $\left|b^{\prime}\right| \geq \omega^{3}$ and principal for addition, clearly $f$ is total recursive. Finally, let $d^{\prime}=3 \cdot 5^{d}$ in $\mathcal{O}$ where, for each $n, d_{n}=g\left(b_{f(n)}\right)$. It is evident that, for all $n, d_{n} \leq_{0} b_{f(n)}$; for every $i, \delta\left(d_{i-1}, d_{i}\right)$ is a limit notation, and $\left|d^{\prime}\right|=\left|b^{\prime}\right|$. (Since $b^{\prime} \precsim^{\prime}$, it also follows that $b^{\prime} \approx d^{\prime}$.)

The proof of this theorem is based upon a priority argument which is motivated as follows. It is sufficient to construct $c^{\prime}=3 \cdot 5^{c}=\Sigma_{O i=0}^{\infty} \gamma_{i}$, where $\left|\gamma_{i}\right|<\left|b^{\prime}\right|$ for each $i$ and $\gamma_{i}$ 's are enumerated by a recursive $\{e\}(i)$ so that for some strictly increasing recursive function $f^{c}(s)$ and every $s, x$, and $n$, the following three conditions are satisfied:

$(1)_{s}^{c}$

$$
\left|c_{f^{c}(2 s)}\right| \leq\left|b_{f^{c}(2 s)}\right|
$$
there exists an $i$ such that either $\{x\}(i)$ diverges, $\{x\}(i)<i+1$, or $\left|a_{i}\right| \underline{L}\left|c_{\{x\}(i)}\right|$, and

$(3)_{n}^{c}$ there exists a finite sequence of numbers $i_{0}<i_{1}<\cdots<i_{n}$ such that $\left|\gamma_{i_{j}}\right|=\left|\delta\left(b_{j-1}, b_{j}\right)\right|$ for $j=0,1, \ldots, n$.

Likewise, the analogous conditions are to hold for $a^{\prime}=3 \cdot 5^{a}=\Sigma_{O_{i=0}}^{\infty} a_{i}$ and some strictly increasing recursive function $f^{a}(s)$.

Suppose that we have constructed $a^{\prime}$ and $c^{\prime}$ satisfying the above. Conditions (3) $)_{n}^{c}$ and (3) $)_{n}^{a}$ insure that $\left|a^{\prime}\right|=\left|c^{\prime}\right|=\left|b^{\prime}\right|$. Conditions (1) $)_{s}^{a}$ and (1) $)_{s}^{c}$ insure that $a^{\prime} \precsim b^{\prime}$ and $c^{\prime} \precsim b^{\prime}$. Conditions (2) ${ }_{x}^{c}$ insure that $a^{\prime} \not c^{\prime}$ for if $a^{\prime} \precsim c^{\prime}$, then there is a recursive function with Gödel number $x$ such that $\left.\left|a_{i}\right| \leq \mid c_{x}\right\}_{(i)} \mid$; but clearly we can assume $i+1 \leq\{x\}(i)$ for all $i$, contradicting (2) $)_{x}^{c}$. Similarly, conditions $(2)_{x}^{a}$ insure that $c^{\prime} \not a^{\prime}$. But now the theorem easily follows.

Definition 3.1. For each condition $(2)_{x}^{a}$ or $(2)_{x}^{c}$ the conditions occurring to 
its left in the following sequence are said to have bigher priority than that condition: $(2)_{0}^{c},(2)_{0}^{a},(2)_{1}^{c},(2)_{1}^{a}, \cdots,(2)_{x}^{c},(2)_{x}^{a},(2)_{x+1}^{c},(2)_{x+1}^{a}, \cdots$. If condition $y$ has higher priority than condition $z$, we write $z<_{p} y$.

The purpose of this priority assignment is to well-order the most interesting conditions we need to satisfy. In the construction of $a^{\prime}, c^{\prime}$ below we will always attempt to satisfy all conditions $z$ with higher priority than condition $y$ before satisfying condition $y$. However, at any given stage $s$ it is not possible to determine effectively which conditions $z$ have been satisfied. Consequently, we associate certain $E$-criteria with each condition $z$ under consideration at stage $s$ and use freely Theorem 2.2 to control the size of $\gamma_{i}\left(\alpha_{i}\right)$ being defined at that stage. If a condition of higher priority than the one under consideration at stage $s$ has not been met via the $E$-criteria associated with it, then we will learn this fact at some later stage and be able to use the function $q$ of Theorem 2.2(vi) to compute precisely how far astray we have gone.

In our construction we keep track of certain ordered pairs of numbers whose convergence properties are relevant to our priority conditions by means of disjoint sets $F_{s}^{a}$ and $F_{s}^{c}$. If $(x, y) \in F_{s}^{a}\left(F_{s}^{c}\right)$, then the condition it is associated with is $(2)_{x}^{a}\left((2)_{x}^{c}\right)$, often denoted by $(a, x)((c, x))$. We let the priority assignment on $(2)_{x}^{a}$ and $(2)_{x}^{c}$ induce a priority assignment on the elements of $F_{s}^{a} \cup F_{s}^{c}$ in the natural way so that it is meaningful to find an element of highest priority in any nonempty subset of $F_{s}^{a} \cup F_{s}^{c}$. The function $p(s)$ below is used to keep track of what condition we attempt to satisfy at stage $s$ ( $s$ even). The function $f^{c}(s)$ $\left(f^{a}(s)\right)$ defined inductively in the construction is used to keep track of the growth of $c^{\prime}\left(a^{\prime}\right)$ relative to $b^{\prime}$ and will eventually insure $(1)_{s}^{c}\left((1)_{s}^{a}\right)$ for all $s$. The functions $I_{s}^{c}\left(I_{s}^{a}\right)$ are simply bookkeeping functions used to insure $(3)_{n}^{c}\left((3)_{n}^{a}\right)$ for all $n$.

We now give the construction of $a^{\prime}=\Sigma_{O_{i=0}}^{\infty} a_{i}, c^{\prime}=\Sigma_{O_{i=0}}^{\infty} \gamma_{i}$ by stages. At each stage $s$, we define $\alpha_{i}$ in $\mathcal{O}$ for $f^{a}(s-1)<i \leq f^{a}(s)$ and $\gamma_{i}$ in $\Theta$ for $f^{c}(s-1)<$ $i \leq f^{c}(s)$. $D_{s}^{a}$ and $D_{s}^{c}$ will hold certain numbers which represent conditions that we have assumed satisfied at some stage $2 s^{\prime}+1 \leq s$; namely if $x \in D_{s}^{a}$, then we have assumed at some stage $2 s^{\prime}+1 \leq s$ that all conditions of priority higher or equal to that of $(2)_{x}^{c}$ have been satisfied before stage $2 s^{\prime}+1$.

Stage 0. $I_{0}^{a}(x)=x, I_{0}^{c}(x)=x$ for all $x, p(0)=(c, 0), F_{0}^{c}=\{(0,0)\}, \alpha_{0}=$ $F\left(r((c, 0), 0), \delta\left(b_{-1}, b_{0}\right)\right)$ where $\{r((c, 0), 0)\}(t)=0$ for all $t, \gamma_{0}=2$, and $f^{a}(0)=$ $f^{c}(0)=0 . D_{0}^{a}=D_{0}^{c}=F_{0}^{a}=G_{0}^{a}=G_{0}^{c}=\varnothing$.

Convention. $D_{s}^{a}, D_{s}^{c}, F_{s}^{a}, F_{s}^{c}, G_{s}^{a}, G_{s}^{c}, I_{s}^{a}, I_{s}^{c}$ will be defined to be the same as at stage $s-1$ unless otherwise specified. Also, $f^{a}(s)=f^{a}(s-1)+1, f^{c}(s)=$ $f^{c}(s-1)+1, a_{i}=2$ for $f^{a}(s-1)<i \leq f^{a}(s)$, and $\gamma_{i}=2$ for $f^{c}(s-1)<i \leq f^{c}(s)$ unless specified otherwise. Frequently, we will define $I_{s}^{a}\left(I_{s}^{c}\right)$ at stage $s$ by 
writing $I_{s}^{a}(k)=j$ for some $k$ and $j$ which is to mean $I_{s}^{a}(x)=I_{s-1}^{a}(x)$ if $x<k$ and $l_{s}^{a}(x+k)=x+j$ for $x \geq 0$.

Stage 1. $p(1)=(a, 0), F_{1}^{a}=\{(0,1)\}$.

Convention. If for some $k,(x, k) \in F_{s}^{a}\left(F_{s}^{c}\right)$, then we denote by $x_{s}^{a}\left(x_{s}^{c}\right)$ the largest number $y$ such that $(x, y) \in F_{s}^{a}\left(F_{s}^{c}\right)$.

Stage $2 s(s>0)$. Case 1. Case 1 holds if, for all $x,(a, x) p p(2 s-1)$ and, for some $j,(x, j) \in F_{2 s-1}^{a}$ implies $\urcorner(E t)_{t \leq 2 s} T_{1}\left(x, x_{2 s-1}^{a}, t\right)$; and for all $x$, $(c, x)_{p}>p(2 s-1)$ and, for some $j,(x, j) \in F_{2 s-1}^{c}$ implies

$$
\urcorner(E t)_{t \leq 2 s^{T}} T_{1}\left(x, x_{2 s-1}^{c}, t\right) .
$$

Case 2 holds otherwise.

Case 1, Subcase A. $p(2 s-1)=(a, x)$. Define $p(2 s)=(a, x)$ and $k=f^{c}(2 s)=$ $f^{c}(2 s-1)+1$. Let $j=I_{2 s-1}^{c}(x)$ and define

$$
\gamma_{k}=F\left(r((a, x), 2 s), \delta\left(b_{j-1}, b_{j}\right)\right)
$$

where $F$ is the function of Theorem 2.2 and $r((a, x), 2 s)$ is a Gödel number of the following recursive function $b(t)$ :

$$
\begin{aligned}
& b(t)=0, \quad \text { if }(z)\left(t^{\prime}\right)_{t^{\prime} \leq t}\left(\left\{\left[(a, z)_{p}>(a, x) \wedge(E y)\left((z, y) \in F_{2 s}^{a}\right)\right] \rightarrow \bar{T}_{1}\left(z, z_{2 s}^{a}, t^{\prime}\right)\right\}\right. \\
& \left.\wedge\left\{\left[(c, z)_{p}>(a, x) \wedge(E y)\left((z, y) \in F_{2 s}^{c}\right)\right] \rightarrow \bar{T}_{1}\left(z, z_{2 s}^{c}, t^{\prime}\right)\right\}\right) .
\end{aligned}
$$

(Note that, for all $t, b(t)=0$, iff for each $(a, z)_{p}>(a, x)$ either there is no $j$ such that $(z, j) \in F_{2 s}^{a}$ or there is a $j$ such that $(z, j) \in F_{2 s}^{a}$ and $\{z\}\left(z_{2 s}^{a}\right)$ diverges, and for each $(c, z)_{p}>(a, x)$ either there is no $j$ such that $(z, j) \in F_{2 s}^{c}$ or there is a $j$ such that $(z, j) \in F_{2 s}^{c}$ and $\{z\}\left(z_{2 s}^{c}\right)$ diverges. By Theorem 2.2, if, for all $t$, $b(t)=0$, then we are assured that $\left|\gamma_{f_{(2 s)}}\right|=\left|\delta\left(b_{j-1}, b_{j}\right)\right|$ (this is the only way that the construction insures $\left.(3)_{j}^{c}\left((3)_{j}^{a}\right)\right)$. On the other hand, if, for some $t$, $b(t) \neq 0$, then by Theorem $2.2\left|\gamma_{f}{ }^{c}(2 s)\right|<\omega^{2}$. This has the effect of retarding the growth of $c^{\prime}$ to $\left|b^{\prime}\right|$. When $p(2 s)=(a, x)(p(2 s)=(c, x))$ we are attempting to make $\left|\gamma_{f^{c}(2 s)}\right|\left(\left|\alpha_{f^{c}(2 s)}\right|\right)$ large if all conditions of priority higher than $(a, x)$ $((c, x))$ diverge at stage $2 s$ (Definition 3.4) and $\left|\gamma_{f^{c}(2 s)}\right|<\omega^{2}\left(\left|a_{f^{c}(2 s)}\right|<\omega^{2}\right)$, otherwise. It will follow by iteration of Case 2, Subcase 2 with $p(2 s)=(a, x)$ and all conditions of priority higher than $(a, x)$ diverging at stage $2 s$ that $\left|c_{n}\right|$ will eventually become larger than $\left|a_{\{x\}(n)}\right|$ if $\{x\}$ is a strictly increasing function; thus, assuring condition $(a, x)$, i.e, $(2)_{x}^{a}$. The crucial assumption that $\left|b^{\prime}\right| \geq \omega^{3}$ and $\left|b^{\prime}\right|$ is principal for addition assures us that, for infinitely many $m$, $\left|\delta\left(b_{m-1}, b_{m}\right)\right| \geq \omega^{2}$; this together with the fact that when we discover a $t$ such 
that $\left\{r\left((c, x+1), 2 s^{\prime}\right)\right\}(t) \neq 0$ Theorem 2.2(vi) allows us to compute effectively a number $j$ such that $\left|a_{f^{c}\left(2 s^{\prime}\right)}\right| \leq \omega \cdot j$ become important in comparing at odd stages $2 s^{\prime \prime}+1$ the growth of $a^{\prime}$ and $c^{\prime}$ to $\left|b^{\prime}\right|$.)

Note that in Case 1, Subcase B below, $r((c, x), 2 s)$ is defined by interchanging $a$ and $c$ in the above if $p(2 s)=(c, x)$.

Case 1, Subcase B. $p(2 s-1)=(c, x)$ (here $x>0$ always). Define $p(2 s)=$ $(c, x)$ and $k=f^{a}(2 s)=f^{a}(2 s-1)+1$. Let $j=I_{2 s-1}^{a}(x-1)(x-1$ here rather than $x$ as in Subcase $A$ because $(a, x-1)$ is the condition of lowest priority with priority higher than that of $(c, x))$ and define

$$
a_{k}=F\left(r((c, x), 2 s), \delta\left(b_{j-1}, b_{j}\right)\right) .
$$

Stage $2 s(s>0)$. Case 2. Let $(x, y)$ be the element of highest priority in $F_{2 s-1}^{a}\left(F_{2 s-1}^{c}\right)$ such that $(x, y)=\left(x, x_{2 s-1}^{a}\right)\left((x, y)=\left(x, x_{2 s-1}^{c}\right)\right)$, $(E t)_{t \leq 2 s} T_{1}\left(x, x_{2 s-1}^{a}, t\right)\left((E t)_{t \leq 2 s} T_{1}\left(x, x_{2 s-1}^{c}, t\right)\right)$, and $(a, x)_{p}>p(2 s-1)$ $\left((c, x){ }_{p}>p(2 s-1)\right)$.

Case 2, Subcase A. $(x, y) \in F_{2 s-1}^{c}$ (Subcase B corresponds to $(x, y) \epsilon$ $\left.F_{2 s-1}^{c}\right)$.

Subcase A, Case $A_{1} \cdot x^{\prime} \in D_{2 s-1}^{a} \rightarrow(a, x)<_{p}\left(c, x^{\prime}\right)$ and $x^{\prime} \in D_{2 s-1}^{c} \rightarrow$ $(a, x)<_{p}\left(a, x^{\prime}\right)$.

Case $A_{2}$. If otherwise.

Case $\mathrm{A}_{1}$, Subcase 1. $x \notin G_{2 s-1}^{a}$ and $\{x\}(y)<y+1$. Define $F_{2 s}^{a}=F_{2 s-1}^{a}-$ $\left\{\left(x, y^{\prime}\right): y^{\prime} \in N\right\}$ and set $G_{2 s}^{a}=G_{2 s-1}^{a} \cup\{x\}$. (Here we know for sure that condition $(a, x)$ is satisfied.) Define $p(2 s)=(c, x+1)$ and proceed as in Subcase 3 below.

Case $A_{1}$, Subcase 2. $x \notin G_{2 s-1}^{a}$ and $\{x\}(y) \geq y+1$. Define $F_{2 s}^{a}=F_{2 s-1}^{a} \cup$ $\{(x, z)\}$ where $z$ is the smallest number $k \geq 2 s$ larger than all $j$ such that $\left(x^{\prime}, j\right) \epsilon$ $F_{s^{\prime}}^{a} \cup F_{s^{\prime}}^{c}$ for some $x^{\prime}$ and some $s^{\prime}<2 s$. Define $p(2 s)=(a, x)$. Let $2 s_{0}$ be the largest $2 s^{\prime}<2 s$ such that $p\left(2 s^{\prime}\right)=(a, x)$ and $(x, y) \in F_{2 s^{\prime}}^{a}$. By Lemmas 3.3 and $3.42 s_{0}$ exists. Let $Q_{c}=\left\{2 s^{\prime}: 2 s>2 s^{\prime}>2 s_{0} \wedge p\left(2 s^{\prime}\right)=\left(a, x^{\prime}\right)<_{p}(a, x)\right\}$. If $Q_{c}=\varnothing$, then define $f^{c}(2 s)=f^{c}(2 s-1)+1$ and $I_{2 s}^{c}(x)=j+1$ where $j=I_{2 s s_{0}}^{c}(x)$. If $Q_{c} \neq \varnothing$, then for $2 s^{\prime} \in Q_{c}$ it is clear that $(E t \leq 2 s)\left(\left\{r\left(p\left(2 s^{\prime}\right), 2 s^{\prime}\right)\right\}(t) \neq 0\right)$ and using Theorem 2.2(vi) and the least such $t$, we can effectively find $k_{2 s^{\prime}}$ such that $\left|\gamma_{k}\right| \leq \omega \cdot k_{2 s^{\prime}}$ for $k=f^{c}\left(2 s^{\prime}\right)$. Define $f^{c}(2 s)=1+\Sigma_{2 s^{\prime} \in Q_{c}} k_{2 s^{\prime}}+f^{c}(2 s-1)$ and $I_{2 s}^{c}(x)=j+1$ where $j=I_{2 s}^{c}(x)$. Finally, define $\gamma_{k}=F\left(r((a, x), 2 s), \delta\left(b_{i-1}, b_{i}\right)\right)$ where $k=f^{c}(2 s)$ and $i=I_{2 s}^{c}(x)$.

Let $Q_{a}=\left\{2 s^{\prime}: 2 s>2 s^{\prime}>2 s_{0} \wedge p\left(2 s^{\prime}\right)=\left(c, x^{\prime}\right)<_{p}(a, x)\right\}$. If $Q_{a}=\varnothing$, then $f^{a}(2 s)=f^{a}(2 s-1)+1$. If $Q_{a} \neq \varnothing$, then for each $2 s^{\prime} \in Q_{a},(E t \leq 2 s)\left(\left\{r\left(p\left(2 s^{\prime}\right)\right.\right.\right.$, $\left.\left.2 s^{\prime}\right)\right\}(t) \neq 0$ ) and using Theorem $2.2(\mathrm{vi})$ and the least such $t$ we can effectively find $k_{2 s^{\prime}}$ such that $\left|a_{k}\right| \leq \omega \cdot k_{2 s^{\prime}}$ for $k=f^{a}\left(2 s^{\prime}\right)$. Define $f^{a}(2 s)=$ 
$1+\Sigma_{2 s^{\prime} \in Q_{a}}{ }^{k} 2 s^{\prime}+f^{a}(2 s-1)$. Finally, define $l_{2 s}^{a}(x)=j$ where $j=l_{2 s^{\prime}}^{a}(x)$ and $2 s^{\prime}$ is the smallest element of $Q_{a}$ such that $p\left(2 s^{\prime}\right)=(c, x+1)$, or $I_{2 s}^{a}=I_{2 s-1}^{a}$, otherwise.

Case $A_{1}$, Subcase 3. $x \in G_{2 s-1}^{a}$ and $(x, y) \in F_{2 s-1}^{a}$. Define $p(2 s)=$ $(c, x+1), F_{2 s}^{a}=F_{2 s-1}^{a}-\{(x, y)\}$. Define $D_{2 s}^{a}=D_{2 s-1}^{a}-\{x\}$, if $\{x\}(y)<y+1$, or $D_{2 s}^{a}=D_{2 s-1}^{a}$ otherwise. Let $2 s_{0}$ be the largest $2 s^{\prime}<2 s$ such that $p\left(2 s_{0}\right)=$ $(a, x)$ and $(x, y) \in F_{2 s-1}^{a}$. Let $Q_{a}, Q_{c}, f^{a}(2 s)$, and $f^{c}(2 s)$ be defined exactly as in Subcase 2. $I_{2 s}^{a}(x)=j$ where $j=I_{2 s_{1}}^{a}(x)$ where $2 s_{1}$ is the smallest element of $Q_{a}$ such that $p\left(2 s_{1}\right)=(c, x+1)$, or $I_{2 s}^{a}=I_{2 s-1}^{a}$, otherwise. Define $a_{k}=$ $F\left(r((c, x+1), 2 s), \delta\left(b_{i-1}, b_{i}\right)\right)$ where $k=f^{a}(2 s)$ and $i=I_{2 s}^{a}(x) . I_{2 s}^{c}(x+1)=j$ where $j=I_{2 s^{\prime}}^{c}(x+1)$ and $2 s^{\prime}$ is the smallest element of $Q_{c}$ such that $p\left(2 s^{\prime}\right)=$ $(a, x+1)$, or $I_{2 s}^{c}=I_{2 s-1}^{c}$, otherwise.

Stage $2 s$, Subcase A, Case $A_{2} \cdot(x, y) \in F_{2 s-1}^{a}$ and either there is an $x^{\prime} \epsilon$ $D_{2 s-1}^{c}$ such that $(a, x) \underset{p-}{\geq}\left(a, x^{\prime}\right)$ or there is an $x^{\prime} \in D_{2 s-1}^{a}$ such that $(a, x) \geq\left(c, x^{\prime}\right)$.

Let $S^{a}=\left\{z: z \in D_{2 s-1}^{a}\right.$ and $\left.(c, z) \leq_{p}(a, x)\right\}$. For $z \in S^{a}$, define inductively $D_{z}=\left\{(z, y):(z, y) \in F_{s^{\prime}}^{a}\right.$ for some $\left.s^{\prime}<2 s\right\}$ if, for some $j,(z, j) \in F_{2 s-1}^{a}$.

$D_{z}=\left\{(z, y):(z, y) \in F_{s^{\prime}}^{a}\right.$ for some $\left.s^{\prime}<2 s\right\} \cup\{(z, k)\}$ where $k$ is the smallest $y \geq 2 s$ such that $y$ is larger than all $j$ such that, for some $v,(v, j)$ belonged to $F_{s^{\prime}}^{a} \cup F_{s^{\prime}}^{c}$ with $s^{\prime}<2 s$ or to $D_{z^{\prime}}$ for some $z^{\prime}<z$ and $z^{\prime} \in S^{a}$, otherwise.

Let $S^{c}=\left\{z: z \in D_{2 s-1}^{c}\right.$ and $\left.(a, z) \leq_{p}(a, x)\right\}$. For $z \in S^{c}$, define inductively

$E_{z}=\left\{(z+1, y):(z+1, y) \in F_{s^{\prime}}^{c}\right.$ for some $\left.s^{\prime}<2 s\right\}$ if, for some $j,(z+1, j) \epsilon$ $F_{2 s-1}^{c}$

$E_{z}=\left\{(z+1, y):(z+1, y) \in F_{s^{\prime}}^{c}\right.$ for some $\left.s^{\prime}<2 s\right\} \cup\{(z+1, k)\}$ where $k$ is the least number $y \geq 2 s$ larger than all $j$ such that for some $v(v, j)$ belonged to $F_{s^{\prime}}^{a} \cup F_{s^{\prime}}^{c}$ with $s^{\prime}<2 s$, to $D_{z}$ with $z \in S^{a}$, or to $E_{z^{\prime}}$ for some $z^{\prime} \in S^{c}$ and $z^{\prime}<z$, otherwise.

Replace:

$$
\begin{array}{ll}
F_{2 s-1}^{c} \text { by } & F_{2 s-1}^{c} \cup \underset{z \in S^{c}}{\bigcup} E_{z}, \\
D_{2 s-1}^{c} \text { by } D_{2 s-1}^{c}-S^{c}, \\
G_{2 s-1}^{c} \text { by } D_{2 s-1}^{c}-\left\{z+1: z \in S^{c}\right\} .
\end{array}
$$

and

$$
\begin{aligned}
& F_{2 s-1}^{a} \text { by } F_{2 s-1}^{a} \cup \bigcup_{z \in S^{a}} D_{z}, \\
& D_{2 s-1}^{a} \text { by } D_{2 s-1}^{a}-s^{a}, \\
& G_{2 s-1}^{a} \text { by } G_{2 s-1}^{a}-s^{a} .
\end{aligned}
$$


With these replacements, we start at stage $2 s$ anew. Now, it is clear since $(x, y) \in F_{2 s-1}^{a}$ that Stage $2 s$, Case 2, Subcase $A$, Case $A_{1}$ applies and proceed as specified there with $(x, y)$ being the element of highest priority insuring

\section{Case 2.}

Stage $2 s$, Case 2 , Subcase B. $(x, y) \in F_{2 s-1}^{c}$. The construction is exactly analogous to the above but the analogous priority assignments must be strictly followed, i.e., it is the same except for the variation between the priority assignments on $a$ and $c$ obtained by interchanging $a$ and $c, a$ and $\gamma, f^{a}$ and $f^{c}$, etc. Thus, we do not explicitly write out the details.

Stage $2 s+1$. If $p(2 s)=(c, z)(p(2 s)=(a, z))$, then $p(2 s+1)=(a, z)$ $(p(2 s+1)=(c, z+1))$. If $2 s+1$ is the first stage $s^{\prime}$ for which $p\left(s^{\prime}\right)=(a, z)$ $\left(p\left(s^{\prime}\right)=(c, z+1)\right)$, then $F_{2 s+1}^{a}=F_{2 s}^{a} \cup\{(z, j)\}\left(F_{2 s+1}^{c}=F_{2 s}^{c} \cup\{(z+1, j)\}\right)$ where $j$ is the smallest number larger than $2 s+1$ such that $j$ has never occurred previously as a second coordinate of any element of $F_{s^{\prime}}^{a} \cup F_{s^{\prime}}^{c}$ with $s^{\prime}<2 s+1$. If $p(2 s)=(a, z)((c, z))$ and Case 1 below holds for $(a, z)((c, z))$, then $F_{2 s+1}^{a}$ $\left(F_{2 s+1}^{c}\right)$ is defined as specified there (note there is no conflict here since we are defining $F_{2 s+1}^{c}\left(F_{2 s+1}^{a}\right)$ first, then defining $F_{2 s+1}^{a}\left(F_{2 s+1}^{c}\right)$ next).

Case 1. Suppose $p(2 s)=(a, x)$ (analogously, if $p(2 s)=(c, x)$ ). Let $2 s_{0}$ be the largest $2 s^{\prime}<2 s$ such that $p\left(2 s^{\prime}\right)=(c, x)$ (by Lemma $3.4,2 s_{0}$ exists). Next let $2 s_{1}$ be the largest $2 s^{\prime}$ with $2 s_{0} \leq 2 s^{\prime} \leq 2 s$ such that, for some $j,(x, j)$ is removed from $F_{2 s^{\prime}}^{c}$, i.e., for some $j,(x, j) \in F_{2 s^{\prime}-1}^{c}$ and $(x, j) \notin F_{2 s^{\prime}}^{c}$, or $2 s_{1}=$ $2 s_{0}$, otherwise. In order to determine if Case 1 holds at stage $2 s+1$ for every two elements $\left(x, k_{1}\right)$ and $\left(x, k_{2}\right)$ in $F_{2 s}^{a}$ with $k_{1}<k_{2}$ and $f^{a}\left(2 s_{0}\right) \leq k_{2}$ we carry out the following procedure:

Suppose there is a number $j$ such that $f^{a}\left(2 s_{0}\right) \leq j$ and for some $2 s_{2}$ with $2 s_{1} \leq 2 s_{2} \leq 2 s, p\left(2 s_{2}\right)=(a, x), j=I_{2 s_{2}}^{c}(x), f^{c}\left(2 s_{2}\right) \leq k_{1}$, and

$$
(t)_{t \leq 2 s+1}^{(*)}\left(\left\{r\left((c, x), 2 s_{0}\right)\right\}(t)=0 \quad \text { and } \quad\left\{r\left((a, x), 2 s_{2}\right)\right\}(t)=0\right)
$$

otherwise, Case 1 does not hold for this choice of $\left(x, k_{1}\right)$ and $\left(x, k_{2}\right)$ in $F_{2 s}^{a}$. Let $j^{*}$ and $2 s_{2}$ denote the smallest numbers satisfying the above, choosing $j^{*}$ first.

If the above does hold for $\left(x, k_{1}\right)$ and $\left(x, k_{2}\right)$, then in order for Case 1 to hold for them we next require that $f^{a}(2 s) \geq k_{2}$ and for every $2 s^{\prime}$ with $2 s_{0}<2 s^{\prime}<$ $2 s$ such that $p\left(2 s^{\prime}\right)=(c, w)$ (by the above choice of $2 s_{0}$ and Lemma 3.4, $\left.(c, w)<_{p}(a, x)\right)$ and $f^{a}\left(2 s_{0}\right)<f^{a}\left(2 s^{\prime}\right) \leq k_{2}$, there exists a $t \leq 2 s+1$ such that $\left\{r\left((c, w), 2 s^{\prime}\right)\right\}(t) \neq 0$. Using Theorem 2.2 (vi) we can compute effectively for each $2 s^{\prime}$ as above and the least $t$ such that $\left\{r\left((c, w), 2 s^{\prime}\right)\right\}(t) \neq 0$ a number $q_{2 s^{\prime}}$ such that $\left|\alpha_{f^{a}\left(2 s^{\prime}\right)}\right| \leq \omega \cdot q_{2 s^{\prime}}$. Thus, 


$$
\left|\delta\left(a_{f^{a}\left(2 s_{0}\right)}, a_{k_{2}}\right)\right|=\left|\sum_{i=f^{a}\left(2 s_{0}\right)+1}^{k_{2}} a_{i}\right| \leq\left(\omega \cdot\left(\sum_{2 s^{\prime} \in S^{2}} q_{2 s^{\prime}}\right)\right)+\omega
$$

where $S=\left\{2 s^{\prime}: 2 s_{0}<2 s^{\prime}<2 s, p\left(2 s^{\prime}\right)=(c, w)\right.$, and $\left.f^{a}\left(2 s^{\prime}\right) \leq k_{2}\right\}$ since, for any $i$ with $f^{a}\left(2 s_{0}\right)+1 \leq i \leq k_{2},\left|a_{i}\right|=1$ unless $i=f^{a}\left(2 s^{\prime}\right)$ for some $2 s^{\prime} \in S$. Let $m=$ $\left(\Sigma_{2 s^{\prime} \in S} q_{2 s^{\prime}}\right)+1$.

It follows (by Lemma 3.8) that $\left|a_{f^{a}\left(2 s_{0}\right)}\right| \leq\left|b_{f^{a}\left(2 s_{0}\right)}\right|$. Also, by our choice of $2 s_{2}\left|c_{c_{\left(2 s_{2}\right)}}\right| \leq\left|c_{k_{1}}\right|$ and (by Lemma 3.1(iii)) $\{x\}\left(k_{1}^{0}\right)$ converges and $k_{1}<$ $\{x\}\left(k_{1}\right)<k_{2}$. Next by Theorem 1.1 compute $\operatorname{enm}(d, 0)$, enm $(d, 1), \cdots$, enm $(d, 2 s+1)$ where $d=\delta\left(c_{f^{c}}{ }_{\left(2 s_{2}\right)}, c_{k_{1}}\right)$ and let $m^{*}$ be the number of distinct numbers of the form $3 \cdot 5^{y}$ which occur in this list. If $m^{*}>m$, then we say that for this choice of $\left(x, k_{1}\right)$ and $\left(x, k_{2}\right)$ Case 1 holds. Case 1 holds at stage $2 s+1$ if for some choice of $\left(x, k_{1}\right)$ and $\left(x, k_{2}\right)$ in $F_{2 s}^{a}$ Case 1 holds, i.e., the above conditions are all satisfied.

Suppose Case 1 holds for condition $(a, x)((c, x))$. Define $D_{2 s+1}^{a}=D_{2 s}^{a} \cup$ $\{x\}$ and $G_{2 s+1}^{a}=G_{2 s}^{a} \cup\{x\}\left(D_{2 s+1}^{c}=D_{2 s}^{c} \cup\{x-1\}\right.$ and $\left.G_{2 s+1}^{c}=G_{2 s}^{c} \cup\{x\}\right)$. Let $k$ be the largest $y$ such that $(x, y) \in F_{2 s}^{a}\left(F_{2 s}^{c}\right)$. Define $F_{2 s+1}^{a}=F_{2 s}^{a}-\{(x, y)$ : $y \neq k\}\left(F_{2 s+1}^{c}=F_{2 s}^{c}-\{(x, y): y \neq k\}\right)$.

[This paragraph is to motivate what is done at stage $2 s+1$, Case 1 . Suppose for $\left(x, k_{1}\right)$ and $\left(x, k_{2}\right)$ in $F_{2 s}^{a}$, Case 1 holds at stage $2 s+1$. If $(*)$ holds for all $t \geq 2 s+1$ as well as $t \leq 2 s+1$ (which of course we cannot determine effectively), then by our inductive argument of Lemma 3.14 it will follow that $\left|b_{f^{a}\left(2 s_{0}\right)}\right| \leq\left|c_{f^{c}\left(2 s_{2}\right)}\right|$. ((*) holds for all $t \geq 2 s+1$ as well as for $t \leq 2 s+1$ is equivalent to the property that at stage $2 s_{0}$ all elements of priority higher than $(c, x)$ diverge and that at stage $2 s_{2}$ all elements of priority higher than $(a, x)$ diverge (Definition 3.4).) Suppose that $\left|c_{k_{1}}\right| \leq\left|a_{\{x\}\left(k_{1}\right)}\right|$; then Case 1 assures us that

$$
\left|a_{f_{\left(2 s_{0}\right)}}\right| \leq\left|b_{f^{a}\left(2 s_{0}\right)}\right| \leq\left|c_{f_{\left(2 s_{2}\right)}}\right| \leq\left|c_{k_{1}}\right| \leq\left|a_{\{x\}\left(k_{1}\right)}\right|<\left|a_{k_{2}}\right|
$$

but it then follows that

$$
\left|\delta\left(a_{f} a_{\left(2 s_{0}\right)}, a_{k_{2}}\right)\right| \geq\left|\delta\left(c_{f}{ }_{\left(2 s_{2}\right)}, c_{k_{1}}\right)\right|
$$

and this contradicts the Case 1 computation that $m^{*}>m$. Thus, assuming (*) holds for all $t$, it follows that $\left.\left|c_{k_{1}}\right| \leq \mid a_{\{x}\right\}_{\left(k_{1}\right)} \mid$ is false, i.e., condition $(a, x)$ is true. If $(*)$ does not hold for all $t$, then not all elements of priority higher than $(a, x)$. diverge at stage $2 s_{2}$ or not all elements of priority higher than $(c, x)$ 
diverge at stage $2 s_{0}$ and consequently at some even stage $2 s^{\prime}>2 s+1$, Case 2 , Case $\mathrm{A}_{2}$ or $\mathrm{B}_{2}$ will hold and since $x \in D_{2 s^{\prime}-1}^{a}$ we will correct the construction accordingly knowing that at stage $2 s+1,(a, x)$ need not have been verified. Our priority assignment will assure us that eventually all conditions $(a, x)$ $((c, x))$ become satisfied.]

Case 2. Case 2 holds, if Case 1 does not hold; and we proceed according to our conventions. This completes the construction.

Definition 3.2. We say $(x, y)$ is placed in $F_{s}^{a}\left(F_{s}^{c}\right)$ if $(x, y) \in F_{s}^{a}\left(F_{s}^{c}\right)$ and $(x, y) \notin F_{s-1}^{a}\left(F_{s-1}^{c}\right)$. We say $(x, y)$ is removed from $F_{s}^{a}\left(F_{s}^{c}\right)$ if $(x, y) \in F_{s-1}^{a}$ $\left(F_{s-1}^{c}\right)$ and $(x, y) \notin F_{s}^{a}\left(F_{s}^{c}\right)$. Similarly, $x$ is placed in $G_{s}^{a}\left(G_{s}^{c}, D_{s}^{a}, D_{s}^{c}\right)$ if $x \in G_{s}^{a}$ $\left(G_{s}^{c}, D_{s}^{a}, D_{s}^{c}\right)$ and $x \notin G_{s-1}^{a}\left(G_{s-1}^{c}, D_{s-1}^{a}, D_{s-1}^{c}\right)$ and analogously, for $x$ is removed from $G_{s}^{a}\left(G_{s}^{c}, D_{s}^{a}, D_{s}^{c}\right)$.

The next lemma enumerates some of the basic properties of the above construction and is useful in justifying results about the construction.

Lemma 3.1. (i) If $(x, k)$ is removed from $F_{s}^{a}$, then $(E t)_{t \leq s} T_{1}(x, k, t)$, every $(x, j) \in \bigcup_{i \leq s} F_{i}^{a}$ with $j \leq k$ does not belong to $F_{s}^{a}$, and $x \in G_{s^{\circ}}^{a}$

(ii) If $(x, k)$ is placed in $F_{s}^{a}$, then every $(x, j)$ in $U_{i<s} F_{s}^{a}$ also belongs to $F_{s}^{a}, x \notin G_{s}^{a}, x \notin D_{s}^{a}, p(s), p(a, x)$, and if $(x, k) \in \bigcup_{i<s} F_{i}^{a}$, then there is a $y>k$ such that $(x, y) \in F_{s}^{a}$.

(iii) If $s$ is the first stage such that $(x, k)$ is placed in $F_{s}^{a}$, then $s \leq k$, for every $(x, j) \in \bigcup_{i<s} F_{i}^{a}\left((E t)_{t \leq s} T_{1}(x, j, t)\right)$ and $j+1 \leq\{x\}(j)<s, p(s){ }_{p} \geq(a, x)$, and $(x, k)$ is the only element of the form $(x, j)$ in $F_{s}^{a}-\bigcup_{i<s} F_{i}^{a}$.

(iv) If $s$ is a stage such that $x \in G_{s}^{a}$ and $x \notin D_{s}^{a}$ (while $x \notin G_{s-1}^{a}$ or $\left.x \in D_{s-1}^{a}\right)$, then for all $j,(x, j) \notin F_{s}^{a}$ and for some $(x, y) \in \bigcup_{i<s} F_{i}^{a},\{x\}(y)<y+1$.

(v) If $x$ is placed in $G_{s}^{a}$, then $s$ is odd implies $x$ is placed in $D_{s}^{a}$ and there is a unique element of the form $(x, j)$ in $F_{s}^{a}$, and $s$ is even implies $x \notin D_{s}^{a}$ and there is no element of the form $(x, j)$ in $F_{s}^{a}$.

(vi) If $x$ is placed in $D_{s}^{a}$, then $s$ is odd and $x$ is placed in $G_{s}^{a}$.

(vii) If $x$ is removed from $G_{s}^{a}$, then $x \in D_{s-1}^{a}, p(s){ }_{p} \geq(a, x), s$ is even, Case 2 , Case $\mathrm{A}_{2}$ or $\mathrm{B}_{2}$ applies at stage $s$, and $x$ is removed from $D_{s}^{a}$.

(The analogous results bold for $F_{s}^{c}, G_{s}^{c}$, and $D_{s}^{c}$ where in formulation one replaces $x \notin D_{s}^{a}, x \in D_{s}^{a}$, and $x$ is removed from $D_{s}^{a}$ by $(x-1) \notin D_{s}^{c},(x-1) \in D_{s}^{c}$, $x-1$ is placed in $D_{s}^{c}$, and $x-1$ is removed from $D_{s}^{c}$, respectively.)

Proof. By induction on $s$, we establish (i)-(vii) simultaneously. The results are clear for $s=0$ and $s=1$. Moreover, we need only verify any of (i)-(vii) at stage $s$ in case its individual hypothesis is satisfied. Suppose (i)-(vii) hold for all $s^{\prime}<s$, and suppose $s$ is even. If Case 1 holds at stage $s$, then by our convention $F_{s}^{a}, G_{s}^{a}$, and $D_{s}^{a}$ remain the same as at stage $s-1$ and (i)-(vii) hold. 
Suppose $s$ is even and Case 2 holds at stage $s$. Suppose Subcase A, Case $A_{1}$ or Case $A_{2}$ is applicable, i.e., $(x, y)$ as in Case 2 with $(x, y) \in F_{s-1}^{a}$ and $(E t)_{t \leq s^{2}} T_{1}(x, y, t)$. Let $s^{\prime}<s$ be the first stage such that $(x, y) \in F_{s^{\prime}}^{a-1}$ By (iii) at stage $s^{\prime}$ it follows that if $(x, j) \in \bigcup_{i<s^{\prime}} F_{i}^{a}$, then $y>j$ and, by (ii) at $s^{\prime}$, $(x, j) \in F_{s^{\prime}}^{a}$. It will follow by our inductive hypothesis that if $(x, j) \in \bigcup_{i<s} F_{i}^{a}$, then $(x, j) \in F_{s^{\prime}}^{a}$. For suppose $(x, j) \in \bigcup_{i<s} F_{i}^{a}$ and $(x, j) \notin F_{s^{\prime}}^{a}$. Suppose $(x, j)$ is first placed in $F_{s^{\prime \prime}}^{a}$ where $s^{\prime}<s^{\prime \prime}<s$. By (ii) at $s^{\prime \prime}$, it follows that $(x, y) \in F_{s^{\prime \prime}}^{a}$ and by (iii) at $s^{\prime \prime}, y<j$. Since $y$ is the largest number $k$ such that $(x, k) \in F_{s-1}^{a}$ by Case 2 conditions, $(x, j)$ must be removed from $F_{s_{2}}^{a}$ for some $s_{2}, s^{\prime \prime}<s_{2} \leq s-1$ and we assume $s_{2} \leq s-1$ is the largest number such that $(x, j)$ is removed from $F_{s_{2}}^{a}$. By (i) at stage $s_{2},(x, y) \notin F_{s_{2}}^{a}$. Consequently, $(x, y)$ must be placed in $F_{s_{3}}^{a}$ for some $s_{3}, s_{2}<s_{3} \leq s-1$ but then by (ii) at stage $s_{3},(x, j) \in F_{s_{3}}^{a}$, contrary to our choice of $s_{2}$. Thus, $(x, j) \in F_{s^{\prime}}^{a}$. In addition, it follows that $(x, y)$ belongs to $F_{s^{\prime \prime}}^{a}$ for all $s^{\prime \prime}, s^{\prime} \leq s^{\prime \prime} \leq s-1$, since if $(x, y)$ is removed from $F_{s^{\prime \prime}}^{a}$, $s^{\prime}<s^{\prime \prime}<s-1$, then it must be placed in $F_{s^{\prime \prime \prime}}^{a}, s^{\prime \prime}<s^{\prime \prime \prime} \leq s-1$, for some $s^{\prime \prime \prime}$, and by the last part of (ii) at $s^{\prime \prime \prime}$, there is a $k>y$ such that $(x, k) \in F_{s^{\prime \prime \prime}}^{a}$. By the above, $(x, k) \in F_{s^{\prime}}^{a}$ contrary to (iii) at $s^{\prime}$. Hence, for all $s^{\prime \prime}, s^{\prime} \leq s^{\prime \prime} \leq s-1$, $\left(x, x_{s^{\prime \prime}}^{a}\right)=(x, y) \in F_{s^{\prime \prime}}^{a}$. We restate this result as Lemma 3.3 below.

Suppose now at stage $s$, Case $A_{1}$, Subcase 1 holds, then all elements of the form $(x, j)$ are removed from $F_{s}^{a}$ and $x$ is placed in $G_{s}^{a}$. Thus (i) holds for $k=y$ and for $(x, j) \in F_{s-1}^{a}, j \neq y$, (i) holds by (iii) at $s^{\prime}$ since $(x, j) \in \bigcup_{i<s^{\prime}} F_{i}^{a}$ by the above. The hypothesis of (iv) holds since $x \notin D_{s-1}^{a}$ (and $x \notin D_{s}^{a}$ ), for otherwise, let $s_{1}$ be the largest $s^{\prime \prime}$ such that $x$ is placed in $D_{s^{\prime \prime}}^{a}$ with $s^{\prime \prime} \leq s-1$. By (vi) at $s_{1}, x$ is placed in $G_{s_{1}}^{a}$. Since $x \notin G_{s-1}^{a}$, there is an $s_{2}$ such that $s_{1}<$ $s_{2} \leq s-1$ and $x$ is removed from $G_{s_{2}}^{a}$. By (vii) at $s_{2}, x$ is removed from $D_{s_{2}}^{a}$ contrary to the choice of $s_{1}$. Clearly, the conclusion of (iv) holds since $\{x\}(y)<y+1$. Clearly, (v) holds since $x \notin D_{s}^{a}$ by the above and there are no elements of the form $(x, j)$ in $F_{s}^{a}$.

Suppose now at stage $s$, Case $A_{1}$, Subcase 2 holds, then $s$ is the first stage such that $(x, z)$ is placed in $F_{s}^{a}$ and $y+1 \leq\{x\}(y)<s \leq z$ by the choice of $z$ and the fact that $\{x\}(y)<t$ if $T_{1}(x, y, t)$. If $(x, j) \in \bigcup_{i<s} F_{i}^{a}$ and $j \neq y$, then by the above $(x, j) \in \bigcup_{i<s^{\prime}} F_{i}^{a}$ where $(x, y)$ was first placed in $F_{s^{\prime}}^{a}$. Consequently, the remaining parts of (iii) follow from (iii) at $s^{\prime}$ and also $p(s)=(a, x)$. Since $x \notin$ $D_{s-1}^{a}$ by the arguments for (iv) given above in Subcase 1 , (ii) follows if we show every $(x, j)$ in $\bigcup_{i<s} F_{i}^{a}$ belongs to $F_{s}^{a}$. Suppose $(x, j) \in \bigcup_{i<s} F_{i}^{a}$, then $(x, j) \epsilon$ $F_{s^{\prime}}^{a}$. It will follow that $(x, j) \in F_{s-1}^{a}$, for suppose $(x, j) \notin F_{s-1}^{a}$, and let $s_{1}$ denote the largest $s^{\prime \prime} \leq s-1$ such that $(x, j)$ is removed from $F_{s^{\prime \prime}}^{a}$. By (i) at $s_{1}$, $x \in G_{s_{1}}^{a}$. Since $x \notin G_{s-1}^{a}$, it follows that $x$ must be removed from $G_{s_{2}}^{a}$ for some 
$s_{2}, s_{1}<s_{2} \leq s-1$, and by (vii) since Case 2, Case $A_{2}$ or $\mathrm{B}_{2}$ applies at stage $s_{2}$ all elements of the form $(x, k)$ in $\bigcup_{i<s_{2}} F_{i}^{a}$ also belong to $F_{s_{2}}^{a}$ contrary to our choice of $s_{1}$. Thus, $(x, j) \in F_{s-1}^{a}$ and consequently $(x, j) \in F_{s^{\circ}}^{a}$ This establishes (i)-(vii) at stage $s$.

Suppose now at stage $s$, Case 2, Case $A_{1}$, Subcase 3 holds. Let $s^{\prime}$ be the first stage such that $(x, y) \in F_{s^{\prime}}^{a}$. From the above $(x, y) \in F_{s^{\prime \prime}}^{a}$ for all $s^{\prime \prime}, s^{\prime} \leq$ $s^{\prime \prime} \leq s-1$, and any $(x, j) \in \bigcup_{i<s} F_{i}^{a}$ also belongs to $F_{s^{\prime}}^{a}$. It follows that $x \in$ $D_{s-1}^{a}$, for suppose $x \notin D_{s-1}^{a}$, and let $s_{1}$ denote the largest $s^{\prime \prime} \leq s-1$ such that $x$ is placed in $G_{s^{\prime \prime}}^{a}$. By (iii) at $s^{\prime}$, it follows that $s^{\prime}<s_{1}$ and by (v), $s_{1}$ is odd since $(x, y) \in F_{s_{1}}^{a}$ and $x$ is placed in $D_{s_{1}}^{a}$. If $x$ is removed from $D_{s^{\prime \prime}}^{a}$ for some $s^{\prime \prime}$, $s_{1}<s^{\prime \prime} \leq s-1$, it follows by (iv) at $s^{\prime \prime}$ since $x \in G_{s^{\prime \prime}}^{a}$ that $(x, y) \notin F_{s^{\prime \prime}}^{a}$, contrary to the above. Thus, $x \in D_{s-1}^{a}$. Suppose now $\{x\}(y)<y+1$, then at stage $s, x$ is removed from $D_{s}^{a}$ and $x \in G_{s}^{a}$, also $(x, y)$ is removed from $F_{s}^{a}$. It follows that (iv) holds since, by $(v)$ at $s_{1},(x, y)$ is the only element of the form $(x, j)$ in $F_{s_{1}}^{a}$ and no element of the form $(x, j)$ can be placed in $F_{s^{\prime \prime}}^{a}$ for $s_{1}<s^{\prime \prime} \leq s-1$ since otherwise, by (ii), $x \notin G_{s^{\prime \prime}}^{a}$, contrary to the choice of $s_{1}$. Since $(x, y)$ is the only element removed from $F_{s}^{a}$, (i) follows at stage $s$. Suppose now that $\{x\}(y) \geq y+1$. Then $(x, y)$ is removed from $F_{s}^{a}$ but $x$ is not removed from $D_{s}^{a}$. As above, (i) holds. Thus, (i)-(vii) hold at stage $s$.

Suppose at stage $s$, Subcase $A$, Case $A_{2}$ holds, i.e., for $(x, y)$ as in Case 2, $(x, y) \in F_{s-1}^{a},\{x\}(y)$ converges, and for some $x^{\prime} \in D_{s-1}^{a},(a, x){ }_{p} \geq\left(c, x^{\prime}\right)$ or for some $x^{\prime} \in D_{s-1}^{c},(a, x)_{p} \geq\left(a, x^{\prime}\right)$. Suppose $x^{\prime} \in D_{s-1}^{a}$ and $(a, x)_{p} \geq\left(c, x^{\prime}\right)$, i.e., $x<x^{\prime}$. It follows by the inductive hypothesis that $x^{\prime} \in G_{s-1}^{a}$ since if $s_{1}$ is the largest stage $s^{\prime \prime} \leq s-1$ such that $x^{\prime}$ is placed in $D_{s^{\prime \prime}}^{a}$, it follows by (vi) that $x^{\prime}$ is placed in $G_{s}^{a}$ and if $x^{\prime}$ is removed from $G_{s^{\prime \prime}}^{a}$ for some $s_{1}<s^{\prime \prime} \leq s-1$, it follows by (vii) that $x^{\prime}$ is removed from $D_{s^{\prime \prime}}^{a}$, contrary to our choice of $s_{1}$. Thus, $x^{\prime} \epsilon$ $G_{s-1}^{a}$ and $s_{1}$ is the largest $s^{\prime \prime}$ such that $s^{\prime \prime} \leq s-1$ and $x^{\prime}$ is placed in $G_{s^{\prime \prime}}^{a}$. No $\left(x^{\prime}, j\right)$ is placed in $F_{s^{\prime \prime}}^{a}$ for $s_{1}<s^{\prime \prime} \leq s-1$ by (ii), and by (v) $s_{1}$ is odd and there is exactly one element $\left(x^{\prime}, k\right)$ in $F_{s_{1}}^{\bar{a}}$. If $\left(x^{\prime}, k\right) \in F_{s-1}^{a}$, then let $s_{0}$ denote the first stage such that $\left(x^{\prime}, k\right) \in F_{s_{0}}^{a}$. By the results in the second paragraph of this proof, it follows that every $\left(x^{\prime}, j\right) \in \bigcup_{i<s} F_{i}^{a}$ belongs to $F_{s_{0}}^{a}$. The construction now places all $\left(x^{\prime}, j\right) \in \bigcup_{i<s} F_{i}^{a}$ in $F_{s}^{a}$, removes $x^{\prime}$ from $G_{s^{\prime}}^{a^{\prime}}$, and removes $x^{\prime}$ from $D_{s^{\circ}}^{a}$. It will follow below that $p(s){ }_{p} \geq(c, x+1)_{p}>\left(a, x^{\prime}\right)$ and, clearly, (ii) holds for $\left(x^{\prime}, j\right)$ placed in $F_{s}^{a}$. Moreover, (vii) holds for $x^{\prime}$. If $\left(x^{\prime}, k\right) \notin F_{s-1}^{a}$, then an $\left(x^{\prime}, z\right)$ is placed for the first time in $F_{s}^{a}$ along with all elements $\left(x^{\prime}, j\right)$ such that $\left(x^{\prime}, j\right) \in \bigcup_{i<s} F_{i}^{a}, x^{\prime}$ is removed from $G_{s}^{a}$, and $x^{\prime}$ is removed from $D_{s}^{a}$. Let $s_{2}$ denote the largest $s^{\prime \prime} \leq s-1$ such that $\left(x^{\prime}, k\right)$ is removed from $F_{s^{\prime \prime}}^{a}$, then $s_{1}<s_{2}$ and there is exactly one element of the form $\left(x^{\prime}, j\right)$ in $F_{s_{2}-1}^{a}$ and hence 
$s_{2}$ is even. Since $x^{\prime} \in G_{s_{2}-1}^{a}$, it follows that Case 2 , Case $A_{1}$ or $A_{2}$, Subcase 3 applies at stage $s_{2}$ and since $x^{\prime} \in D_{s_{2}}^{a}$, it follows that $\left\{x^{\prime}\right\}(k) \geq k+1$ and $(E t)_{t \leq s_{2}} T_{1}\left(x^{\prime}, k, t\right)$. Thus, (iii) and (ii) hold since $p(s) \geq_{p}(c, x+1) \geq_{p}\left(a, x^{\prime}\right)$ since $x^{\prime}>x$ and $z \geq s$. Clearly (vii) holds. Note in case $x^{\prime} \in D_{s-1}^{c}$ and $(a, x)_{p} \geq\left(a, x^{\prime}\right)$, we now replace $\left(x^{\prime}+1, j\right)$ in $F_{s}^{c}$, etc. as above first, i.e., the Case. $\mathrm{A}_{2}$ or $\mathrm{B}_{2}$ part of stage $s$ does not interfere with $(x, j)$ 's in $F_{s}^{a}$ where $(x, y) \in F_{s-1}^{a}$ is the $(x, y)$ of Case 2. The proof of the next part of this stage breaks up into the Subcase 1, Subcase 2, and Subcase 3 as viewed above and, consequently, is treated exactly as above.

Suppose now $s$ is odd. If $s$ is the smallest number such that $p(s)=\left(a, x^{\prime}\right)$, then an element $\left(x^{\prime}, k\right)$ is placed in $F_{s}^{a}$ for the first time and there are no elements of the form $\left(x^{\prime}, j\right)$ in $\bigcup_{i<s} F_{i}^{a}$. Thus, (ii) holds since clearly $x^{\prime} \notin G_{s-1}^{a}$ and $x^{\prime} \notin D_{s-1}^{a}$ since in order for $x^{\prime}$ to be placed in $G_{s^{\prime}}^{a}$ or $D_{s^{\prime}}^{a}$, there must be at least one element of the form $\left(x^{\prime}, j\right)$ in $F_{s^{\prime}-1^{a}}^{a}$. Likewise (iii) holds. Suppose now that Case 1 holds for $(a, x)$ at stage $s, s$ odd, then for the $x$ of that case all elements of the form $(x, j)$ in $F_{s-1}^{a}$ except $\left(x, x_{s-1}^{a}\right)$ are removed from $F_{s}^{a}$. At stage $s, x$ is placed in $G_{s}^{a}$ since if $x \in G_{s-1}^{a}$, let $s_{1}$ denote the largest stage $s^{\prime} \leq s-1$ such that $x$ is placed in $G_{s^{\prime}}^{a}$. By $(v), s_{1}$ must be odd since otherwise there is no element of the form $(x, j)$ in $F_{s_{1}}^{a}$ and hence at some $s^{\prime \prime}, s_{1}<s^{\prime \prime} \leq$ $s-1,(x, j)$ must be placed in $F_{s^{\prime \prime}}^{a}$ and by (ii) $x \notin G_{s^{\prime \prime}}^{a}$, contrary to our choice of $s_{1}$. Thus, $s_{1}$ must be odd and there is exactly one element of the form $(x, k)$ of $F_{s_{1}}^{a}$; however, there must be at least two elements of the form $(x, j)$ in $F_{s-1}^{a}$, and, hence, by (ii), $x \notin G_{s^{\prime \prime}}^{a}$ for some $s_{1}<s^{\prime \prime} \leq s-1$, contrary to our choice of $s_{1}$. Thus, $x \notin G_{s-1}^{a}$ and $x$ is placed in $G_{s}^{a}$. Similarly, $x$ is placed in $D_{s}^{a}$ since $x \notin D_{s-1}^{a}$; otherwise let $s_{1}$ be the largest stage such that $x$ is placed in $D_{s^{\prime \prime}}^{a}$, $s^{\prime \prime} \leq s-1$. By (vi), $x$ is placed in $G_{s_{1}}^{a}$ and $s$ is odd. Hence, there is a single element of the form $(x, k)$ in $F_{s_{1}}^{a}$ by (v). Again by (ii), $x \notin D_{s^{\prime \prime}}^{a}$ for some $s_{1}<$ $s^{\prime \prime} \leq s-1$, contrary to the choice of $s_{1}$. Thus, $x$ is placed in $D_{s}^{a}$. Clearly, (v) holds and (vi) holds at stage $s$. Also, (i) holds by our inductive hypothesis at stage $s^{\prime}$ where $\left(x, x_{s-1}^{a}\right)$ is first placed in $F_{s^{\prime}}^{a}$ at stage $s^{\prime}$ and the usual argument, that no elements of the form $(x, j)$ are first placed in $F_{s^{\prime \prime}}^{a}$ for $s^{\prime}<s^{\prime \prime} \leq$ $s-1$. Q.E.D.

Lemma 3.2. If, at stage $s, x \in G_{s}^{a}$ and $x \notin D_{s}^{a}$, then for all $s^{\prime} \geq s$ and all $j$, $(x, j) \notin F_{s^{\prime}}^{a}, x \in G_{s^{\prime}}^{a}, x \notin D_{s^{\prime}}^{a}$, and $(2)_{x}^{a}$ is true.

Proof. Assume $s$ is the smallest $s^{\prime}$ such that $x \in G_{s^{\prime}}^{a}$ and $x \notin D_{s^{\prime}}^{a}$. By (iv), $(2)_{x}^{a}$ holds and for all $j,(x, j) \notin F_{s}^{a}$. It is clear that no $(x, j)$ is placed in $F_{s^{\prime}}^{a}$ for $s^{\prime}$ odd and $s^{\prime} \geq s$. Because, in order for $x$ to be placed in $G_{s^{\prime \prime}}^{a}, s^{\prime \prime} \leq s$, there 
must be at least one element of the form $(x, y)$ in $F_{s^{\prime \prime}-1}^{a}$, consequently the first odd stage $s_{1}$ such that $p\left(s_{1}\right)=(a, x)$ has occurred prior to stage $s^{\prime \prime}$ and this is the only odd stage where an element $(x, j)$ is placed in $F_{s_{1}}^{a}$. Suppose now that the result is true for all $s^{\prime \prime}, s \leq s^{\prime \prime}<s^{\prime}$. Suppose $(x, j)$ is placed in $F_{s^{\prime}}^{a}$; then $s^{\prime}$ is even and Case 2 holds. Since there is no element of the form $(x, k)$ in $F_{s^{\prime}-1}^{a}$, it follows that Case 2, Case $A_{2}$ or $B_{2}$ occurs at stage $s^{\prime}$; but then in order for $(x, j)$ to be placed in $F_{s^{\prime}}^{a}$ it must be that $x \in D_{s^{\prime}-1}^{a}$, contrary to hypothesis. Suppose $x$ is placed in $D_{s^{\prime}}^{a}$; then by Lemma $3.1(v i) s^{\prime}$ is odd and $x \notin G_{s^{\prime}-1}^{a}$, contrary to hypothesis. Suppose $x$ is removed from $G_{s^{\prime}}^{a}$, then by Lemma 3.1(vii) $x \in D_{s^{\prime}-1}^{a}$, contrary to hypothesis. Q.E.D.

We restate a result which occurs in the proof of Lemma 3.1.

Lemma 3.3. If Case 2 bolds at stage $2 s$ with $(x, y) \in F_{2 s-1}^{a}$ being the $(x, y)$ of the construction and $s^{\prime}$ is the smallest number sucb that $(x, y) \in F_{s^{\prime}}^{a}$, then $\left(x, x_{s^{\prime \prime}}^{a}\right)=(x, y) \in F_{s^{\prime \prime}}^{a}$ for all $s^{\prime \prime}$ with $s^{\prime} \leq s^{\prime \prime} \leq 2 s-1$. Similarly, for c replacing $a$.

Lemma 3.4. For all $s, p(2 s+1)<_{p} p(2 s)$ and $p(2 s+2){ }_{p} \geq p(2 s+1)$. If $s<s^{\prime}$ and $p\left(2 s^{\prime}\right)<_{p} p(2 s)$, then for any condition $d=(a, x)$ or $d=(c, x)$ sucb that $p\left(2 s^{\prime}\right)<_{p} d<_{p} p(2 s)$ there is an $s^{\prime \prime}$ with $s<s^{\prime \prime}<s^{\prime}$ sucb that $p\left(2 s^{\prime \prime}\right)=d$.

Proof. By the construction $p(2 s+1)$ is always the condition of highest priority lower than that of $p(2 s)$. Also, $p(2 s), p p(2 s-1)$ since in Case 1, stage $2 s, p(2 s)=p(2 s-1)$ and in Case 2 , Subcase $A$ at stage $2 s$ with $(x, y)$ the $(x, y)$ of the construction $(a, x){ }_{p}>p(2 s-1)$ and either $p(2 s)=(c, x+1)_{p} \geq p(2 s-1)$ (Subcase 1 or Subcase 3 ) or $p(2 s)=(a, x)$ (Subcase 2); similarly if Case 2, Subcase $B$ holds at stage $2 s+1$.

The second assertion is now obvious.

Below $I_{2 s}^{a}(-1)$ is to be interpreted by 0 .

Definition 3.3. We say $x$ is secured at stage $2 s$ for $a(c)$ and $(c, z)((a, z))$ secures $x$ for $a(c)$ at stage $2 s$ if at stage $2 s$,

$$
\begin{aligned}
& p(2 s)=(c, z), I_{2 s}^{a}(z-1)=x, \alpha_{f^{a}(2 s)}=F\left(r((c, z), 2 s), \delta\left(b_{x-1}, b_{x}\right)\right), \\
& \text { and for all } t(\{r((c, z), 2 s)\}(t)=0) \\
& \left(p(2 s)=(a, z), I_{2 s}^{c}(z)=x, \gamma_{f}{ }^{c}(2 s)=F\left(r((a, z), 2 s), \delta\left(b_{x-1}, b_{x}\right)\right),\right. \\
& \text { and for all } t(\{r((a, z), 2 s)\}(t)=0)) .
\end{aligned}
$$

We say $x$ is secured for $a(c)$ by $(c, z)((a, z))$ if there is a stage $2 s$ such that $x$ is secured at stage $2 s$ for $a(c)$ and $p(2 s)=(c, z)(p(2 s)=(a, z))$. 
For the next definition recall from the construction that $z_{s}^{a}\left(z_{s}^{c}\right)$; denote for given $z$ the largest number $y$ such that $(z, y) \in F_{s}^{a}\left(F_{s}^{c}\right)$ if, for some $j,(z, j) \in F_{s}^{a}$ (for some $\left.j,(z, j) \in F_{s}^{c}\right)$.

Definition 3.4. We say at stage $s$ that all elements of priority bigher than $(c, x)$ diverge if $p(s) \leq_{p}(c, x)$, for every $(c, z){ }_{p}>(c, x)$ such that, for some $j(z, j) \in F_{s}^{c},\{z\}\left(z_{s}^{c}\right)$ diverges and for every $(a, z)_{p}>(c, x)$ such that for some $j(z, j) \in F_{s}^{a},\{z\}\left(z_{s}^{a}\right)$ diverges. At stage $s$ the notion of all elements of priority higher than $(a, x)$ diverge is defined similarly.

Lemma 3.5. If $i$ is secured by $(c, z)$ for a at stage $2 s$, then $\left|a_{f^{a}(2 s)}\right|=$ $\left|\delta\left(b_{i-1}, b_{i}\right)\right|$. At stage $2 s,(c, z)$ secures $i$ for a iff $p(2 s)=(c, z), l_{2 s}^{a}(z-1)=i$, and at stage $2 s$ all elements of priority bigher than $(c, z)$ diverge. The analogous result bolds for $(a, z)$.

Proof. Suppose $i$ is secured by $(c, z)$ for $a$ at stage 2s. By Definition 3.3, $a_{f^{a}(2 s)}=F\left(r(p(2 s), 2 s), \delta\left(b_{i-1}, b_{i}\right)\right)$ where $p(2 s)=(c, z)$ and $r((c, z), 2 s)$ is a Gödel number of a recursive function such that for all $t(\{r((c, z), 2 s)\}(t)=0)$. Consequently, by Theorem 2.2(ii)

$$
\left|F\left(r((c, z), 2 s), \delta\left(b_{i-1}, b_{i}\right)\right)\right|=\left|\delta\left(b_{i-1}, b_{i}\right)\right| \text {. }
$$

However, by definition, $\{r((c, z), 2 s)\}(t)=0$ iff

$$
\begin{aligned}
& (x)\left(t^{\prime}\right)_{t^{\prime} \leq t}\left(\left\{\left[(a, x){ }_{p}>(c, z) \text { and }(E y)\left((x, y) \in F_{2 s}^{a}\right)\right] \rightarrow \bar{T}_{1}\left(x, x_{2 s^{\prime}}^{a}, t^{\prime}\right)\right\}\right. \\
& \left.\quad \text { and }\left\{\left[(c, x)_{p}>(c, z) \text { and }(E y)\left((x, y) \in F_{2 s}^{c}\right)\right] \rightarrow \bar{T}_{1}\left(x, x_{2 s^{c}}^{c}, t^{\prime}\right)\right\}\right) .
\end{aligned}
$$

Since for all $t,\{r((c, z), 2 s)\}(t)=0$, it is clear that all elements of priority higher than $(c, z)$ diverge.

Conversely, suppose $p(2 s)=(c, z), I_{2 s}^{a}(z-1)=i$, and at stage $2 s$ all elements of priority higher than $(c, z)$ diverge. By the construction $a_{f^{a}(2 s)}=$ $F\left(r((c, z), 2 s), \delta\left(b_{i-1}, b_{i}\right)\right)$ and by the above for each $t\{r((c, z), 2 s)\}(t)=0$. Consequently, $(c, z)$ secures $i$ for $a$ at stage $2 s$.

For the next lemma recall the convention in the construction that when we define $l_{2 s}^{a}(x)=j$, this means for all $k<x, l_{2 s}^{a}(k)=l_{2 s-1}^{a}(k)$ and for all $k \geq x$, $l_{2 s}^{a}(k)=j+(k-x)$.

Lemma 3.6. If $s_{0}<s, p(2 s)<_{p} p\left(2 s_{0}\right)=(a, x)$, and, for all $s^{\prime}$ with $s_{0}<s^{\prime} \leq s, p\left(2 s^{\prime}\right)<_{p}(a, x)$, then for all $k \leq x I_{2 s}^{c}(k)=I_{2 s_{0}}^{c}(k)$. If $s_{0}<s$, $p(2 s)<_{p}\left(2 s_{0}\right)=(c, x+1)$, and, for all $s^{\prime}$ with $s_{0}<s^{\prime} \leq s, p\left(2 s^{\prime}\right)<_{p}(c, x+1)$, then for all $k \leq x, l_{2 s_{0}}^{a}(k)=I_{2 s}^{a}(k)$. 
Proof. In the construction the value of $I_{2 s^{\prime}}^{c}$ at $k$ changes at $2 s^{\prime}$ from the value of $I_{2 s^{\prime}-2}^{c}$ at $k$ only if Case 2 holds and $p\left(2 s^{\prime}\right){ }_{p} \geq(a, k)$, and the value of $I_{2 s^{\prime}}^{a}$ at $k$ changes from the value of $I_{2 s^{\prime}-2}^{a}$ at $k$ only if Case 2 holds and $p\left(2 s^{\prime}\right)_{p} \geq(c, k+1)$. For suppose at stage $2 s^{\prime}$ Case 2, Subcase A (Subcase B) holds. If $p\left(2 s^{\prime}\right)=(a, x)\left(p\left(2 s^{\prime}\right)=(c, x)\right)$, corresponding to Subcase 2 , then, for some $j, j^{\prime}, I_{2 s^{\prime}}^{c}(x)=j$ and $I_{2 s^{\prime}}^{a}(x)=j^{\prime}$ (for some $j, j^{\prime}, I_{2 s^{\prime}}^{a}(x-1)=j$ and $\left.I_{2 s^{\prime}}^{c}(x)=j^{\prime}\right)$. If $p\left(2 s^{\prime}\right)=(c, x)\left(p\left(2 s^{\prime}\right)=(a, x)\right)$, corresponding to Subcase 1 or Subcase 3 , then for some $j, j^{\prime} I_{2 s^{\prime}}^{c}(x)=j$ and $I_{2 s^{\prime}}^{a}(x-1)=j^{\prime}$ (for some $j, j^{\prime} I_{2 s^{\prime}}^{a}(x)=j$ and $\left.I_{2 s^{\prime}}^{c}(x)=j^{\prime}\right)$. Suppose $I_{2 s^{\prime}}^{c}(k) \neq I_{2 s^{\prime}-2}^{c}(k)$, thus $p\left(2 s^{\prime}\right)=$ $(a, z)$ implies $z \geq k$ and $p\left(2 s^{\prime}\right)=(c, z)$ implies $z \geq k$; hence $p\left(2 s^{\prime}\right) p(a, k)$ by Definition 3.1. Suppose $I_{2 s^{\prime}}^{a}(k) \neq I_{2 s^{\prime}-2}^{a}(k)$, then $p\left(2 s^{\prime}\right)=(c, z)$ implies $k \geq z-1$ and $p\left(2 s^{\prime}\right)=(a, z)$ implies $z \geq k$; hence $p\left(2 s^{\prime}\right)_{p} \geq(c, k+1)$ by Definition 3.1.

The lemma follows easily from the above facts.

A fundamental result concerning the construction is the following

Lemma 3.7. For any stage $2 s$, let $2 s^{c}$ be the largest $2 s^{\prime} \leq 2 s$ such that $p\left(2 s^{\prime}\right)=(a, x)$ where $(a, x)$ is the condition of lowest priority such that $(a, x){ }_{p} \geq p(2 s)$. Let $y_{s}^{c}=I_{2 s^{c}}^{c}(x)$, and define, for $j \leq y_{s^{\prime}}^{c}, k^{c}(2 s, j)=$ largest $2 s^{\prime} \leq 2 s$ such that for some $x^{\prime} p\left(2 s^{\prime}\right)=\left(a, x^{\prime}\right)$ and $j=I_{2 s^{\prime}}^{c}\left(x^{\prime}\right)$. Then

$$
k^{c}(2 s, 0)<k^{c}(2 s, 1)<\ldots<k^{c}\left(2 s, y_{s}^{c}\right)=2 s^{c}, \quad I_{2 s}^{c}=I_{2 s}^{c},
$$

and

$$
\left|c_{f^{c}(2 s)}\right| \leq\left(\sum_{i=0}^{y_{s}}\left|F\left(r\left(p\left(2 s_{i}\right), 2 s_{i}\right), \delta\left(b_{i-1}, b_{i}\right)\right)\right|\right)+\omega \cdot n_{s}^{c}
$$

where $2 s_{i}=k^{c}(2 s, i)$ and $n_{s}^{c} \leq f^{c}(2 s)-y_{s}^{c}$. Similarly, for a. Also, $I_{2 s}^{c}$ and $l_{2 s}^{a}$ are always increasing functions of $x, p(2 s)=\left(a, x^{\prime}\right)$ implies for some $j_{1}, j_{2}$ that $I_{2 s}^{c}\left(x^{\prime}\right)=j_{1}$ and $I_{2 s}^{a}\left(x^{\prime}\right)=j_{2}$, and $p(2 s)=\left(c, x^{\prime}\right)$ implies for some $j_{1}, j_{2}$ that $I_{2 s}^{c}\left(x^{\prime}\right)=j_{1}$ and $I_{2 s}^{a}\left(x^{\prime}-1\right)=j_{2}\left(\right.$ if $\left.x^{\prime}=0, I_{2 s}^{a}\left(x^{\prime}\right)=j_{2}\right)$. (Recall our convention about writing $I_{2 s}^{c}\left(x^{\prime}\right)=j_{1}$. $)$

Proof. These results are easily checked for $s=0$. Suppose the results are true for all $2 s^{\prime}<2 s$. Suppose at stage $2 s$ Case 1 holds and suppose $p(2 s)=$ $(a, x)$, then since $p(2 s)=p(2 s-1)$ it follows by Lemma 3.4 that $p(2 \cdot(s-1))=$ $(c, x)$ and, hence, $p\left(2(s-1)^{c}\right)=(a, x-1)$. Thus, by the inductive hypothesis $I_{2(s-1)}^{c}(x-1)=j_{1}$ for some $j_{1}$ and $I_{2 \cdot(s-1)}^{c}=I_{2(s-1)^{c}}^{c}$, and, hence, since $I_{2 s}^{c}=$ $I_{2 \cdot(s-1)}^{c}$ for Case $1, I_{2 s}^{c}(x)=I_{2(s-1)^{c}}^{c}(x)=j_{1}+1=y_{s-1}^{c}+1$. Thus, $2 s^{c}=2 s$ and $y_{s}^{c}=y_{s-1}^{c}+1$. Also $k^{c}(2 s, j)=k^{c}(2(s-1), j)$ for $j<y_{s}^{c}$ since $p(2 s)=(a, x)$. By our inductive hypothesis, we obtain $k^{c}(2 s, 0)<\cdots<k^{c}\left(2 s, y_{s}^{c}\right)=2 s^{c}$ and also, 


$$
\begin{aligned}
\mid c_{f}{ }^{c}(2 s) & =\left|c_{f^{c}(2(s-1))}\right|+1+\left|\gamma_{f^{c}(2 s)}\right| \\
\leq & \left(\sum_{i=0}^{y_{s-1}^{c}}\left|F\left(r\left(p\left(2 s_{i}\right), 2 s_{i}\right), \delta\left(b_{i-1}, b_{i}\right)\right)\right|\right)+\omega \cdot n_{s-1}^{c}+1 \\
& +\left|F\left(r((a, x), 2 s), \delta\left(b_{y_{s}^{c-1}}^{c}, b_{y_{s}}\right)\right)\right|
\end{aligned}
$$

where $2 s_{i}=k^{c}(2 s, i)$ for $i<y_{s}^{c}$. The last term is a limit ordinal greater than or equal to $\omega$ by Theorem $2.2(\mathrm{v})$ since $\left|\delta\left(b_{i-1}, b_{i}\right)\right|$ is a limit by our initial assumption about $b^{\prime}$. Clearly, if $\omega \leq \alpha$ a limit, then $\left(\omega \cdot n_{s-1}^{c}+1\right)+\alpha \leq \alpha+\left(\omega \cdot n_{s-1}^{c}\right)$ (consider the Cantor Normal Form for $a$ [0]). Thus, letting $n_{s}^{c}=n_{s-1}^{c}$ the result follows. Clearly, $I_{2 s}^{c} c=I_{2 s}^{c}, I_{2 s}^{c}\left(I_{2 s}^{a}\right)$ is increasing, and $p(2 s)=(a, x)$ implies $I_{2 s}^{c}(x)=j_{1}$ where $j_{1}^{2 s}=y_{s}^{c}$ and $l_{2 s}^{a}(x)=j_{2}$ where $j_{2}=I_{2(s-1)}^{a}(x)$ and $l_{2(s-1)}^{a}(x-1)=$ $j_{2}-1$. The result is easy if Case 1 holds and $p(2 s)=(c, x)$, for then $p(2(s-1))=$ $(a, x-1), 2 s^{c}=2(s-1)^{c}, y_{s-1}^{c}=y_{s}^{c}, f^{c}(2 s)=f^{c}(2 \cdot(s-1))+2$, and let $n_{s}^{c}=$ $n_{s \sim 1}^{c}+1$.

Suppose at stage $2 s$, Case 2 , Subcase $A$, Case $A_{1}$, Subcase 2 holds with $p(2 s)=(a, x)$ and since $p(2 s-1)<_{p}(a, x)$, we have $p(2 s-2) \leq_{p}(a, x)$. Let $2 s_{0}$ be the largest $2 s^{\prime}<2 s$ such that the $(x, y)$ of Case 2 belongs to $F_{2 s^{\prime}}^{a}$ and $p\left(2 s^{\prime}\right)=(a, x)$. In order to see that $2 s_{0}$ exists, let $s_{1}$ be the stage at which $(x, y)$ is first placed in $F_{s}^{a}$; by Lemma 3.1 (iii) it follows that $p\left(s_{1}\right) p_{p} \geq(a, x)$ and by Lemma 3.3, $(x, y) \in F_{s^{\prime}}^{a^{1}}$ and $\left(x, x_{s^{\prime}}^{a}\right)=(x, y)$ for every $s^{\prime}, s_{1} \leq s^{\prime} \leq 2 s-1$.

By Lemma 3.4, there is a $2 s^{\prime}$ such that $s_{1} \leq 2 s^{\prime} \leq 2 s-1$ and $p\left(2 s^{\prime}\right)=(a, x)$ since $p(2 s-1)<_{p}(a, x)$; consequently, $p\left(2 s^{\prime}\right)=(a, x)$ and $(x, y) \in F_{2 s^{\prime}}^{a}$. Thus, $2 s_{0}$ exists. Let

$$
Q_{c}=\left\{2 s^{\prime}: 2 s>2 s^{\prime}>2 s_{0} \wedge p\left(2 s^{\prime}\right)=\left(a, x^{\prime}\right)<_{p}(a, x)\right\}_{0}
$$

Suppose $Q_{c}=\varnothing$. Thus, $2(s-1)^{c}=2 s_{0}$ since otherwise $2 s_{0}<2(s-1)^{c} \leq 2(s-1)$ and $\left(a, x^{\prime}\right)=p\left(2(s-1)^{c}\right){ }_{p} \geq p(2 \cdot(s-1))$ while by Lemma 3.4 $p\left(2(s-1)^{c}\right) \leq_{p}(a, x)$; by the definition of $2 s_{0}, p\left(2(s-1)^{c}\right) \neq(a, x)$ and thus $p\left(2(s-1)^{c}\right)<_{p}(a, x)$, contrary to $Q_{c}=\varnothing$. Thus, $2(s-1)^{c}=2 s_{0}$ and by definition $I_{2 s}^{c}(x)=j+1$ where $j=I_{2 s_{0}}^{c}(x)=y_{s-1}^{c}$, since $I_{2 s_{0}}^{c}(x)=j$ by the inductive hypothesis, it follows that $I_{2 s}^{c}$ is increasing. Clearly, $2 s^{c}=2 s, y_{s}^{c}=y_{s-1}^{c}+1$, and $k^{c}\left(2 s, y_{s}^{c}-1\right)=k^{c}\left(2 \cdot(s-1), y_{s-1}^{c}\right)=2(s-1)^{c}=2 s_{0}$. Thus, for $j<y_{s}^{c}$, $k^{c}(2 s, j)=k^{c}(2(s-1), j)$. By our inductive hypothesis, the result follows as above. Suppose $Q_{c} \nRightarrow \varnothing$. By Lemma 3.6, it follows that $I_{2 s_{0}}^{c}(k)=I_{2 s-1}^{c}(k)$ for all $k \leq x$ since for all $s^{\prime}, s_{0}<s^{\prime} \leq s-1, p\left(2 s^{\prime}\right)<_{p}(a, x)$. Since $I_{2 s}^{c}(x)=j+1$ where $j=I_{2 s_{0}}^{c}(x)$, it follows that $I_{2 s}^{c}$ is increasing since $I_{2 s_{0}}^{c}(x)=j$ and is 
increasing, i.e., for $k<x, I_{2 s}^{c}(k)=I_{2 s_{0}}^{c}(k)$ and for $z \geq x, I_{2 s}^{c}(z)=I_{2 s_{0}}^{c}(z)+1$. For $2 s^{\prime} \in Q_{c}$, we have $\left|\gamma_{k}\right| \leq \omega \cdot k_{2 s^{\prime}}$ for $k=f^{c}\left(2 s^{\prime}\right)$ since $(x, y) \in F_{2 s^{\prime}}^{a}$, $p\left(2 s^{\prime}\right)<_{p}(a, x)$, i.e., $\left.(E t)\right)_{t \leq 2 s^{\prime}}\left\{r\left(p\left(2 s^{\prime}\right), 2 s^{\prime}\right)\right\}(t) \neq 0$. Clearly, any $2 s^{\prime}, 2 s_{0}<$ $2 s^{\prime} \leq 2 \cdot(s-1)$ such that for some $x^{\prime} p\left(2 s^{\prime}\right)=\left(a, x^{\prime}\right)$ belongs to $Q_{c}$ by our choice of $2 s_{0}$. We claim $k^{c}\left(2 s, y_{s}^{c}-1\right)=2 s_{0}$ since $p\left(2 s_{0}\right)=(a, x)$ and $I_{2 s_{0}}^{c}(x)=$ $y_{s}^{c}-1$. Suppose for some $2 s^{\prime}, 2 s_{0}<2 s^{\prime} \leq 2 \cdot(s-1), p(2 s)=\left(a, x^{\prime}\right)$ and $I_{2 s^{\prime}}^{c}\left(x^{\prime}\right)=y_{s}^{c}-1$. By Lemma 3.6, we have that $I_{2 s^{\prime}}^{c}(x)=I_{2 s_{0}}^{c}(x)=y_{s}^{c}-1$ but since by inductive hypothesis $I_{2 s^{\prime}}^{c}$ is increasing, it follows that $x=x^{\prime}$, contrary to our choice of $2 s_{0}$. Thus, $k^{c}\left(2 s, y_{s}^{c}-1\right)=2 s_{0}=k^{c}\left(2 s_{0}, y_{s_{0}}^{c}\right)$. By the same argument, $k^{c}(2 s, j)=k\left(2 s_{0}, j\right)$ for any $j \leq y_{s}^{c}-1$. Thus, $k^{c}(2 s, 0)<k^{c}(2 s, 1)<\cdots<$ $k^{c}\left(2 s, y_{s}^{c}\right)=2 s^{c}=2 s$. By our inductive hypothesis at $2 s_{0}$ and the above, it follows that

$$
\begin{aligned}
\left|c_{f^{c}(2 s)}\right| \leq & \sum_{i=0}^{y_{s}^{c}-1}\left|F\left(r\left(p\left(2 s_{i}\right), 2 s_{i}\right), \delta\left(b_{i-1}, b_{i}\right)\right)\right|+\omega \cdot n_{s_{0}}^{c} \\
& +\sum_{2 s^{\prime} \in Q_{c}}\left|\gamma_{f^{c}\left(2 s^{\prime}\right)}\right|+k+\left|F\left(r(p(2 s), 2 s), \delta\left(b_{y_{s}^{c}-1}, b_{y_{s}}\right)\right)\right| \\
\leq & \sum_{i=0}^{y_{s}^{c}-1}\left|F\left(r\left(p\left(2 s_{i}\right), 2 s_{i}\right), \delta\left(b_{i-1}, b_{i}\right)\right)\right|+\omega \cdot n_{s_{0}}^{c} \\
& +\sum_{2 s^{\prime} \in Q_{c}} \omega \cdot k_{2 s^{\prime}}+k+\left|F\left(r(p(2 s), 2 s), \delta\left(b_{y_{s}-1}, b_{y_{s}}\right)\right)\right|,
\end{aligned}
$$

where $2 s_{i}=k^{c}(2 s, i)$ for $i<y_{s}^{c}$. By the argument on ordinals above, we obtain

$$
\left|c_{f^{c}(2 s)}\right| \leq \sum_{i=0}^{y_{s}^{c}}\left|F\left(r\left(p\left(2 s_{i}\right), 2 s_{i}\right), \delta\left(b_{i-1}, b_{i}\right)\right)\right|+\omega \cdot n_{s_{0}}^{c}+\sum_{2 s^{\prime} \in Q_{c}} \omega \cdot k_{2 s^{\prime}} .
$$

Choose $n_{s}^{c}=n_{s_{0}}^{c}+\Sigma_{2 s^{\prime} \in Q_{c}}{ }^{k} 2 s^{\prime}$ and the result follows since $f^{c}(2 s)=1+$ $\Sigma_{2 s^{\prime} \in Q_{c}}{ }^{k} 2 s^{\prime}+f^{c}(2 s-1)$. In order to show $I_{2 s}^{a}$ is increasing and $I_{2 s}^{a}(x)=j_{2}$ for some $j_{2}$, first suppose $Q_{a}=\varnothing$, then $l_{2 s}^{a}=l_{2}^{a} \cdot(s-1)$ and $p(2(s-1)) \leq_{p}(a, x)$. $p(2 \cdot(s-1)) \neq\left(c, x^{\prime}\right)$, for otherwise $\left(c, x^{\prime}\right)<_{p}(a, x)$ and $2(s-1) \in Q_{a}$, contrary to $Q_{a}=\varnothing$. Thus, $p(2(s-1))=\left(a, x^{\prime}\right)$ and if $\left(a, x^{\prime}\right)<_{p}(a, x)$, then there is a $2 s_{0}<2 s^{\prime}<2(s-1)$ such that $p\left(2 s^{\prime}\right)=(c, x+1)$, contrary to hypothesis; consequently, $p(2(s-1))=(a, x)$ and $I_{2(s-1)}^{c}(x)=j_{2}$ for some $j_{2}$, by our inductive hypothesis. Suppose $Q_{a} \neq \varnothing$. By Lemma 3.4, the smallest element $2 s_{1} \in Q_{a}$ is such that $p\left(2 s_{1}\right)=(c, x+1)$ and by the choice of $2 s_{0}$, for all $2 s^{\prime}, 2 s_{0}<2 s^{\prime} \leq$ 
$2 \cdot(s-1), p\left(2 s^{\prime}\right)<_{p}(c, x)$. Consequently, by Lemma 3.6, for all $k \leq x-1$, $I_{2 s_{1}}^{a}(k)=I_{2(s-1)}^{a}(k)$ and, consequently, $I_{2 s}^{a}(x)=j$ where $j=I_{2 s_{1}}^{a}(x)$ implies $I_{2 s}^{a}=$ $l_{2 s_{1}}^{a}$, hence $I_{2 s}^{a}$ is increasing by our inductive hypothesis.

The argument follows the above outline in all the other cases.

Lemma 3.8. For every $s,\left|a_{f^{a}(2 s)}\right| \leq\left|b_{f_{(2 s)}}\right|$ and $\left|c_{f^{c}(2 s)}\right| \leq\left|b_{f^{c}(2 s)}\right|$. Hence, for all $s$, conditions $(1)_{s}^{a}$ and $(1)_{s}^{c}$ are true.

Proof. By Lemma 3.7 we have

$$
\left|a_{f_{(2 s)}}\right| \leq \sum_{i=0}^{y_{s}^{a}}\left|F\left(r\left(p\left(2 s_{i}\right), 2 s_{i}\right), \delta\left(b_{i-1}, b_{i}\right)\right)\right|+\omega \cdot n_{s}^{a},
$$

where $2 s_{i}=k^{a}(2 s, i)$ and $n_{s}^{a} \leq f^{a}(2 s)-y_{s}^{q}$. By Theorem 2.2, we have

$$
\sum_{i=0}^{y_{s}^{a}}\left|F\left(r\left(p\left(2 s_{i}\right), 2 s_{i}\right), \delta\left(b_{i-1}, b_{i}\right)\right)\right| \leq \sum_{i=0}^{y_{s}^{a}}\left|\delta\left(b_{i-1}, b_{i}\right)\right|=\left|b_{y_{s}^{a}}\right| \cdot
$$

However,

$$
\begin{aligned}
\left|b_{f^{a}(2 s)}\right| & =\left|b_{y_{s}^{a}}\right|+\sum_{i=y_{a}^{s+1}}^{f^{(2 s)}}\left|\delta\left(b_{i-1}, b_{i}\right)\right| \\
& \geq\left|b_{y_{s}}\right|+\sum_{i=y_{s}^{a+1}}^{a(2 s)} \omega=\left|b_{y_{s}^{a}}\right|+\omega \cdot \sum_{i=y_{s}^{a+1}}^{a(2 s)} 1 \\
& =\left|b_{y_{s}^{a}}\right|+\omega \cdot\left(f^{a}(2 s)-y_{s}^{a}\right) \geq\left|b_{y_{s}^{a}}\right|+\omega \cdot n_{s}^{a} .
\end{aligned}
$$

Hence, the result follows and similarly for $c$.

Lemma 3.9. If $(a, x)$ secures $k$ for $c$ at stage $2 s$, then for all $s^{\prime}>2 s$, $p\left(s^{\prime}\right) \leq_{p}(a, x)$, no element of the form $(z, j)$ with $(a, z) p_{p}>(a, x)$ is removed or placed in $F_{s^{\prime}}^{a}$, and no element of the form $(z, j)$ with $(c, z){ }_{p}>(a, x)$ is removed or placed in $F_{s^{\prime}}^{c}$. Similarly, if $(c, x)$ secures $k$ for a at stage $2 s$.

Proof. By Lemma 3.5 every element $\left(z, z_{2 s}^{a}\right) \in F_{2 s}^{a}$ such that $(a, z) p_{p}>(a, x)$ has the property that $\{z\}\left(z_{2 s}^{a}\right)$ diverges and every element $\left(z, z_{2 s}^{c}\right) \in F_{2 s}^{c}$ such that $(c, z)_{p}>(a, x)$ has the property that $\{z\}\left(z_{2 s}^{c}\right)$ diverges. By Lemma 3.4 for any condition $(a, z)((c, z))$ of priority higher than $(a, x)$, the first odd stage $s^{\prime \prime}$ such that $p\left(s^{\prime \prime}\right)=(a, z)((c, z))$ occurs before stage $2 s$; thus, no element $(z, j)$ can be placed in $F_{s^{\prime}}^{a}\left(F_{s^{\prime}}^{c}\right)$ if $s^{\prime}$ is odd, $s^{\prime}>2 s$, and $(a, z){ }_{p}>(a, x)$ 
$\left((c, z) p_{p}>(a, x)\right)$. The result for $s^{\prime}$ odd follows inductively since $p\left(s^{\prime}-1\right) \leq_{p}(a, x)$ and at stage $s^{\prime}$ Case 1 holds only for condition $p\left(s^{\prime}-1\right)$; thus, no elements of priority higher than $(a, x)$ can be removed from $F_{s^{\prime}}^{a}\left(F_{s^{\prime}}^{c}\right)$ and $p\left(s^{\prime}\right)<_{p} p\left(s^{\prime}-1\right)$ $\leq_{p}(a, x)$.

Suppose now $s^{\prime}$ is even and that the result holds for all $s^{\prime \prime}, 2 s<s^{\prime \prime}<s^{\prime}$. If Case 1 holds at $s^{\prime}$, then $F_{s^{\prime}}^{a}=F_{s^{\prime}-1}^{a}, F_{s^{\prime}}^{c}=F_{s^{\prime}-1}^{c}$, and $p\left(s^{\prime}\right)=p\left(s^{\prime}-1\right)$; thus, the result holds at $s^{\prime}$. If Case 2 holds at $s^{\prime}$, then let $(u, y)$ in $F_{s^{\prime}-1}^{a} \cup F_{s^{\prime}-1}^{c}$ be the $(x, y)$ of the construction at stage $s^{\prime}$. It follows that $(u, y)=\left(u, u_{s^{\prime}-1}^{a}\right)$ $\left((u, y)=\left(u, u_{s^{\prime}-1}^{c}\right)\right)$ and, hence, $(a, u) \leq_{p}(a, x)\left((c, u) \leq_{p}(a, x)\right)$ since $\{u\}(y)$ converges and the elements $\left(z, z_{s^{\prime}-1}^{a}\right)=\left(z, z_{2 s}^{a}\right)\left(\left(z, z_{s^{\prime}-1}^{c}\right)=\left(z, z_{2 s}^{c}\right)\right),(a, z){ }_{p}>$ $(a, x)\left((c, z){ }_{p}>(a, x)\right)$ have the property that $\{z\}\left(z_{s^{\prime}-1}^{a}\right)\left(\{z\}\left(z_{s^{\prime}-1}^{c}\right)\right)$ diverge. By the construction at Case 2, stage $s^{\prime}$ even, $p\left(s^{\prime}\right)$ has priority the same or lower than that of $(a, u)((c, u))$ and, thus, $p\left(s^{\prime}\right) \leq_{p}(a, x)$. By Lemma 3.1(ii) any element $(z, j)$ placed in $F_{s^{\prime}}^{a}\left(F_{s^{\prime}}^{c}\right)$ must satisfy $(a, z) \leq_{p} p\left(s^{\prime}\right) \leq(a, x)\left((c, z) \leq_{p}\right.$ $\left.p\left(s^{\prime}\right)\right)$; thus, no element of priority higher than $(a, x)$ is placed in $F_{s^{\prime}}^{a}\left(F_{s^{\prime}}^{c}\right)$. An element $(z, j)$ is removed from $F_{s^{\prime}}^{a}\left(F_{s^{\prime}}^{c}\right)$ at even stage $s^{\prime}$ only under Case 2 when $z=u$ with $(z, j),(u, y)$ in $F_{s^{\prime}-1}^{a}\left((z, j),(u, y)\right.$ in $\left.F_{s^{\prime}-1}^{c}\right)$; thus, no element of priority higher than $(a, x)$ can be removed from $F_{s^{\prime}}^{a}\left(F_{s^{\prime}}^{c}\right)$. Q.E.D.

Lemma 3.10. If $k$ is secured for a by $(c, z)$ at stage $2 s_{k}^{a}$, then $\left(c, z^{\prime}\right)$ secures $j$ for a at stage $2 s_{j}^{a}>2 s_{k}^{a}$ implies $\left(c, z^{\prime}\right) \leq_{p}(c, z)$ and $j \geq k+1$. Moreover, if $k$ is secured for a by $(c, z)$ at stage $2 s_{k}^{a}$, then $\left(a, z^{\prime}\right)$ secures $j$ at stage $2 s>2 s_{k}^{a}$ implies $\left(a, z^{\prime}\right) \leq_{p}(c, z)$. The analogous result obtained by interchanging $a$ and $c$ everywhere also bolds.

Proof. Suppose $2 s_{k}^{a}$ is the smallest stage such that for some $z,(c, z)$ secures $k$ for $a$. In particular, $p\left(2 s_{k}^{a}\right)=(c, z)$ and $k=I_{2 s_{k}^{a}}^{a}(z-1)$. By Lemma 3.9, the elements in $F_{s^{\prime}}^{a}\left(F_{s^{\prime}}^{c}\right)$ for $s^{\prime} \geq 2 s_{k}^{a}$ of priority higher than $(c, z)$ are exactly the same elements of priority higher than $(c, z)$ in $F_{2 s_{k}^{a}}^{a}\left(F_{2 s_{k}^{a}}^{a}\right)$. Thus, for all $s^{\prime} \geq 2 s_{k}^{a}$, there does not exist any element $(x, j) \in F_{s^{\prime}}^{a}\left((x, j) \in F_{s^{\prime}}^{c}\right)$ such that $(a, x)_{p}>(c, z)\left((c, x)_{p}>(c, z)\right)$ and $\{x\}\left(x_{s^{\prime}}^{a}\right)$ converges $\left(\{x\}\left(x_{s^{\prime}}^{c}\right)\right.$ converges $)$. Also, for $s^{\prime}>2 s_{k}^{a}, p\left(s^{\prime}\right) \leq_{p}(c, z)$.

Suppose for all $s^{\prime}>2 s_{k}^{a}, p\left(s^{\prime}\right)<_{p}(c, z)$. Let $2 s_{0}$ be the smallest number $2 s^{\prime}>2 s_{k}^{a}$ such that some $j$ is secured for $a$ by some condition $\left(c, z^{\prime}\right)$ at stage $2 s^{\prime}$. By hypothesis $\left(c, z^{\prime}\right)=p\left(2 s_{0}\right)<_{p}(c, z)$ and, hence, $z^{\prime}>z$. By Lemma 3.7, $I_{2 s_{0}}^{a}$ is an increasing function and, consequently, $j=I_{2 s_{0}}^{a}\left(z^{\prime}-1\right)>$ $I_{2 s_{0}}^{a}(z-1)$. By Lemma 3.6, $l_{2 s_{k}^{a}}^{a}(z-1)=I_{2 s_{0}}^{a}(z-1)$. Thus, $j=I_{2 s_{0}}^{a}\left(z^{\prime}-1\right)>$ $l_{2 s_{k}^{a}}^{a}(z-1)=k$. 
Suppose now that there is an $s^{\prime}>2 s_{k}^{a}$ such that $p\left(s^{\prime}\right)=(c, z)$. Let $2 s_{0}$ be the smallest $s^{\prime}>2 s_{k}^{a}$ such that $p\left(s^{\prime}\right)=(c, z)$ ( $s^{\prime}$ is even by Lemma 3.4). We claim $(c, z)$ secures $k+1$ for $a$ at stage $2 s_{0}$ and that $2 s_{0}$ is the first stage $2 s^{\prime}>2 s_{k}^{a}$ such that for some $j, p\left(2 s^{\prime}\right)$ secures $j$ for $a$ at stage $2 s^{\prime}$. Since $p\left(2 s_{0}\right)_{p}>p\left(2 s_{0}-1\right)$, it follows that at stage $2 s_{0}$ Case 2 holds. It follows that the $(x, y)$ of Case 2 is $(z, y) \in F_{2 s_{0}-1}^{c}$ since the $(x, y)$ of Case 2 cannot be of priority higher than $(c, z)$ by the above remarks and if the $(x, y)$ of Case 2 is of lower priority than $(c, z)$, it follows that $p\left(2 s_{0}\right) \leq_{p}$ (priority of $\left.(x, y)\right)<_{p}(c, z)$, contrary to the choice of $2 s_{0}$. By Lemma 3.1(ii), $(z, y) \in F_{2 s_{k}^{a}}^{c}$ By Lemma 3.3, it follows that $(z, y) \in F_{2 s^{\prime}}^{c}$ for all $2 s_{k}^{a} \leq 2 s^{\prime}<2 s_{0}$. Thus, since $\{z\}(y)$ converges, by Lemma 3.5 no $j$ can be secured for $a$ at stage $2 s^{\prime}, 2 s_{k}^{a} \leq 2 s^{\prime}<2 s_{0}$, since $p\left(2 s^{\prime}\right)=\left(c, z^{\prime}\right)<_{p}(c, z)$. By Case 2 conditions in order that $p\left(2 s_{0}\right)=(c, z)$ it follows that Case $B_{1}$, Subcase 2 holds (or first Case $B_{2}$, then Subcase 2 holds). Consequently, $\{z\}(y) \geq(y+1)$ and the largest $2 s^{\prime}<2 s_{0}$ such that $p\left(2 s^{\prime}\right)=(c, z)$ is $2 s_{k}^{a}$. By definition $I_{2 s_{0}}^{a}(z-1)=k+1$ where $k=I_{2 s_{k}^{a}}^{a}(z-1)$ and by Lemmas 3.5 and $3: 9,(c, z)$ secures $k+1$ for $a$ at stage $2 s_{0^{\circ}}$

Suppose now that $2 s$ is the smallest stage larger than $2 s_{k}^{a}$ as above such that $j$ is secured for $c$ by $p(2 s)=\left(a, z^{\prime}\right)$. By Lemma 3.9, $p(2 s)=\left(a, z^{\prime}\right)<_{p}(c, z)$.

By induction the results hold. Q.E.D.

By Lemma 3.10 we obtain immediately the next result.

Lemma 3.11. There is at most one stage $2 s$ such that $k$ is secured for a at stage $2 s$. Moreover, if $k$ is secured for a at stage $2 s_{k}^{a}, j$ is secured for a at stage $2 s_{j}^{a}$, and $k<j$, then $2 s_{k}^{a}<2 s_{j}^{a}$. Similarly, for $c$.

Proof. Let $2 s$ be the first stage at which some $(c, z)$ secures $k$ for $a$. By Lemma 3.10, it follows that only $j \geq k+1$ are secured for $a$ at a later stage $2 s^{\prime}$. Consequently, $k$ is not secured at any stage $2 s^{\prime}>2 s$.

The second result follows by Lemma 3.10 since if $2 s_{j}^{a}>2 s_{k}^{a}$, it follows that $j>k$.

We make the following definition in view of Lemma 3.11.

Definition 3.5. If $k$ is secured for $a$, then the unique stage $2 s$ at which this happens is denoted by $2 s_{k}^{a}$. Similarly, if $k$ is secured for $c$, the unique stage $2 s$ at which this happens is denoted by $2 s_{k}^{c}$.

The following lemma is useful in establishing that every $k$ is secured for $c$ (a).

Lemma 3.12. If $j$ is the largest number secured for $c$ by condition $(a, z-1)$, then for every $2 s \geq 2 s_{j}^{c}$ such that $p(2 s)=(c, z), I_{2 s}^{c}(z)=j+1$. The analogous result bolds for $a$. 
Proof. By Lemma 3.5 at stage $2 s_{j}^{c}$ all elements of priority higher than $(a, z-1)$ diverge. By Lemma 3.9 for all $s^{\prime} \geq 2 s_{j}^{c}, p\left(s^{\prime}\right) \leq_{p}(a, z-1)$ and at stage $s^{\prime} \geq 2 s_{j}^{c}$ all elements of priority higher than $(a, z-1)$ diverge. It follows that, for all $s^{\prime}>2 s_{j}^{c}, p\left(s^{\prime}\right)<_{p}(a, z-1)$; for suppose $s_{0}$ is the smallest $s^{\prime}$ such that $p\left(s^{\prime}\right)_{p} \geq(a, z-1)$ and $s^{\prime}>2 s_{j}^{c}$. By Lemma 3.4, $s_{0}$ must be even and, at stage $s_{0}$, Case 2 holds since if Case 1 holds $p\left(s_{0}\right)=p\left(s_{0}-1\right)<_{p}(a, z-1)$. Since at stage $s_{0}-1$ all elements of priority higher than $(a, z-1)$ diverge and $p\left(s_{0}\right) \geq(a, z-1)$, it follows that the $(x, y)$ of Case 2 must be $(z-1$, $\left.(z-1)_{s_{0}-1}^{a}\right) \in F_{s_{0}-1}^{a}$ and $p\left(s_{0}\right)=(a, z-1)$, i.e., Subcase 2 holds. By Lemma 3.5, $(a, z-1)$ secures $I_{s_{0}}^{c}(z-1)$ for $c$ at stage $s_{0}$, contrary to hypothesis by Lemma 3.10. Thus, $p\left(s^{\prime}\right)<_{p}(a, z-1)$ for all $s^{\prime}>2 s_{j}^{c}$. At stage $2 s_{j}^{c}, j=$ $I_{2 s_{j}^{c}}^{c} c^{(z-1)}$ by Definition 3.3 and by Lemma $3.7 I_{2 s_{j}^{c}}^{c}{ }^{c}(z)=I_{2 s_{j}^{c}}^{c}(z-1)+1=j+1$.

The proof now proceeds by induction on $2 s>2 s_{j}^{c}$ such that $p(2 s)=(c, z)$. There are three possible ways for which $p(2 s)=(c, z)$. The first way is for $p(2 s-1)=(c, z)$ and Case 1 to hold at stage $2 s$; but then since $p(2 s-2)=$ $(a, z-1)$, it follows by the above that $2 s=2 s_{j}^{c}+2$ and, consequently, $l_{2 s}^{c}(z)=$ $I_{2 s}^{c} c(z)=j+1$, by our convention. Note this happens exactly once after stage $2 s_{j}^{c}$.

The second way is for Case 2 to hold at stage $2 s$ with the $(x, y)$ of Case 2 being $\left(z-1,(z-1)_{2 s-1}^{a}\right)$. As in the first paragraph, Subcase 1 or Subcase 3 must hold; it will follow that there are no elements of the form $(z-1, j)$ in $F_{2 s}^{a}$, at stage $2 s$ all elements of priority higher than $(c, z)$ diverge, and, thus, by Lemma 3.9 there is at most one stage $2 s^{\prime}$ where this second alternative takes place. It is clear that if Subcase 1 occurs that there are no elements of the form $(z-1, j)$ in $F_{2 s}^{a}$; so suppose Subcase 3 occurs at stage $2 s$. Since $z-1 \in G_{2 s-1}^{a}$, it follows that $\left(z-1,(z-1)_{2 s-1}^{a}\right)$ is the only element of the form $(z-1, j)$ in $F_{2 s-1}^{a}$. Let $s_{0}$ be the largest stage $s^{\prime} \leq 2 s-1$ such that $z-1$ is placed in $G_{s}^{a}$; by Lemma 3.1(v) $s_{0}$ must be odd, for otherwise by Lemma 3.2 there are no elements of the form $(z-1, j)$ in $F_{2 s-1}^{a}$; hence, there is a single element of the form $(z-1, j)$ in $F_{s_{0}}^{a}$. Let $s_{1}$ be the first stage $s^{\prime}$ such that $\left(z-1,(z-1)_{2 s-1}^{a}\right) \in F_{s^{\prime}}^{a}$ consequently by Lemma 3.1(ii) $z-1 \notin G_{s_{1}}^{a}$ and, hence, $s_{1}<s_{0}$. By Lemma 3.3, $\left(z-1,(z-1)_{2 s-1}^{a}\right) \in F_{s_{0}}^{a}$ and, hence, by Lemma 3.1(ii), $\left(z-1,(z-1)_{2 s-1}^{a}\right)$ is the only element of the form $(z-1, j)$ in $F_{2 s-1}^{a}$. Under Subcase $3,\left(z-1,(z-1)_{2 s-1}^{a}\right)$ is removed from $F_{2 s}^{a}$ and, hence, there are no elements of the form $(z-1, j)$ in $F_{2 s^{\circ}}^{a}$ Now by the construction $I_{2 s}^{c}(z)$ is defined as follows: It is clear that the largest $2 s^{\prime}<2 s$ such that $p\left(2 s^{\prime}\right)=(a, z-1)$ and $\left(z-1,(z-1)_{2 s-1}^{a}\right) \in F_{2 s^{\prime}}^{a}$ is $2 s_{j}^{c}$. Let

$$
Q_{c}=\left\{2 s^{\prime}: 2 s>2 s^{\prime}>2 s_{j}^{c} \wedge p\left(2 s^{\prime}\right)=\left(a, x^{\prime}\right)<_{p}(a, z-1)\right\} .
$$


If $Q_{c}=\varnothing$, then $I_{2 s}^{c}=I_{2 s-1}^{c}$ and, hence, for all $2 s^{\prime}$ such that $2 s_{j}^{c}<2 s^{\prime}<2 s$, it follows by Lemmas 3.4 and 3.2 that $(c, z) \leq_{p} p\left(2 s^{\prime}\right)<_{p}(a, z-1)$. In particular, either $p(2 s-2)=(c, z)$ and then by our inductive hypothesis $I_{2 s}^{c}(z)=I_{2 s-2}^{c}(z)=$ $j+1$, or $p(2 s-2)=(a, z-1)$ and $I_{2 s}^{c}(z)=I_{2 s_{j}^{c}}^{c}(z)=j+1$ by the above.

If $Q_{c} \neq \varnothing$, then let $2 s_{1}$ be the smallest element of $Q_{c}$; by Lemma 3.4 $p\left(2 s_{1}\right)=(a, z)$. By the construction $I_{2 s}^{c}(z)=k$ where $k=I_{2 s}^{c}(z)$. We will show $k=j+1$. We claim $p\left(2 s_{1}-2\right)=(c, z)$. First, by Lemma 3.4 since $p\left(2 s_{j}^{c}\right)=$ $(a, z-1), p\left(2 s_{1}\right)=(a, z)$, and $(a, z-1)_{p}>(c, z){ }_{p}>(a, z)$, there is a $2 s^{\prime}$ such that $2 s_{j}^{c}<2 s^{\prime}<2 s_{1}$ and $p\left(2 s^{\prime}\right)=(c, z)$; let $2 s_{2}$ denote the largest $2 s^{\prime}<2 s_{1}$ such that $p\left(2 s^{\prime}\right)=(c, z)$. By Lemma $3.4 p\left(2 s_{2}+1\right)=(a, z)$ and $p\left(2 s_{2}+2\right) p_{p} \geq$ $(a, z)$. By the hypothesis $p\left(2 s_{2}+2\right)<_{p}(a, z-1)$; hence, $(a, z) \leq_{p} p\left(2 s_{2}+2\right) \leq_{p}$ $(c, z)$ and, by the choice of $2 s_{2}, p\left(2 s_{2}+2\right)=(a, z)$. By the choice of $2 s_{1}, 2 s_{1}=$ $2 s_{2}+2$. Thus, $p\left(2 s_{1}-1\right)=(a, z)$ and $p\left(2 s_{1}\right)=(a, z)$. At stage $2 s_{1}$ either Case 1 holds or Case 2, Subcase B, Subcase 1 or Subcase 3 holds with the $(x, y)$ of Case 2 being $\left(z, z_{2 s_{1}-1}^{c}\right)$. If Case 1 holds at stage $2 s_{1}$, then $I_{2 s_{1}}^{c}=I_{2 s_{1}-2}^{c}$ and by our inductive hypothesis, since $p\left(2 s_{1}-2\right)=(c, z), I_{2 s_{1}-2}^{c}(z)=j+1$; if Case 2 holds at stage $2 s_{1}$, then since the largest stage $2 s^{\prime}<2 s_{1}$ such that $p\left(2 s^{\prime}\right)=(c, z)$ is $2 s_{1}-2$ the $Q_{c}, Q_{a}$ of Case 2 , Subcase B at stage $2 s_{1}$ are both empty; thus, by the construction $I_{2 s_{1}}^{c}=I_{2 s_{1}-2}^{c}$ and by our inductive hypothesis, $I_{2 s_{1}-2}^{c}(z)=j+1$; thus, $k=j+1$.

The third way for which $p(2 s)=(c, z)$ can occur is if at Stage $2 s$, Case 2, Subcase 2 holds with the $(x, y)$ of this case being $\left(z, z_{2 s-1}^{c}\right) \in F_{2 s-1}^{c}$. By the conditions of Case 2 it now follows that $p(2 s-1)<_{p}(c, z)$; by Lemma 3.4 $p(2 s-2) \leq_{p}(c, z)$ and, hence, $2 s-2>2 s_{j}^{c}$. Let $2 s_{0}$ be the largest $2 s^{\prime}<2 s$ such that $p\left(2 s^{\prime}\right)=(c, z)$ and $\left(z,(z)_{2 s-1}^{c}\right) \in F_{2 s^{\prime}}^{c}$; it follows by Lemma 3.3 and Lemma 3.1(iii) (Lemma 3.4) that $2 s_{0}$ exists. Also, $2 s_{0} \geq 2 s_{j}^{c}+2$ and hence by our inductive hypothesis at stage $2 s_{0}, I_{2 s_{0}}^{c}(z)=j+1$. Let

$$
Q_{c}=\left\{2 s^{\prime}: 2 s>2 s^{\prime}>2 s_{0} \wedge p\left(2 s^{\prime}\right)=\left(a, z^{\prime}\right)<_{p}(c, z)\right\} \text {. }
$$

If $Q_{c}=\varnothing$, then $I_{2 s}^{c}=I_{2 s-1}^{c}$ and $I_{2 s}^{c}=I_{2 s-2^{\circ}}^{c}$ However, $p(2 s-2) \leq_{p}(c, z)$ since $p(2 s-1)<_{p}(c, z)$ and, hence, $p(2 s-2)=(c, z)$; for otherwise $p(2 s-2)<_{p}(c, z)$ and since $p\left(2 s_{0}\right)=(c, z), 2 s_{0}<2 s-2$, and by Lemma 3.4, for some $2 s^{\prime}, 2 s_{0}<$ $2 s^{\prime} \leq 2 s-2$, we have $p\left(2 s^{\prime}\right)=(a, z)$ and $Q_{c} \vDash \varnothing$. Thus, $2 s_{0}=2 s-2$ and, hence, $I_{2 s}^{c}(z)=I_{2 s_{0}}^{c}(z)=j+1$. Suppose $Q_{c} \neq \varnothing$, then by the construction let $2 s_{1}$ be the smallest element of $Q_{c}$ such that $p\left(2 s_{1}\right)=(a, z)$ and let $k=I_{2 s_{1}}^{c}(z)$, then $I_{2 s}^{c}(z)=k$. We will show $k=j+1$. Since $2 s_{0}<2 s_{1}<2 s$, it follows that $2 s_{1}=$ $2 s_{0}+2$; for otherwise, $p\left(2 s_{0}+1\right)=(a, z),(c, z)_{p} \geq p\left(2 s_{0}+2\right) p p\left(2 s_{0}+1\right)=$ 
$(a, z)$, and, hence, $p\left(2 s_{0}+2\right)=(c, z)$, contrary to our choice of $2 s_{0}$ by Lemma 3.3. Thus, $2 s_{0}+2=2 s_{1}$ and, hence, since $\left(z,(z)_{2 s-1}^{c}\right) \in F_{2 s_{1}}^{c}$ it follows that Case 1 holds at stage $2 s_{1}$. Thus, $I_{2 s_{1}}^{c}=I_{2 s_{1}-2}^{c}=I_{2 s_{0}}^{c}$. Therefore, $I_{2 s_{1}}^{c}(z)=$ $I_{2 s_{0}}^{c}(z)=j+1$ and, hence, $k=j+1$. Q.E.D.

We state here a fact whose proof is contained in the proof of Lemma 3.12 and finally the last result about the construction.

Lemma 3.13. If $(z, j)$ is removed from $F_{2 s}^{a}$, then there are no elements of the form $(z, k)$ in $F_{2 s^{\circ}}^{a}$

Lemma 3.14. Eacb $k$ is secured for $a(c)$ by some $(c, z)((a, z))$. Eacb condition $(c, z)((a, z))$ secures some $j$ for $a(c)$ and at most finitely many $j$ for $a(c)$. For all $z$, conditions $(c, z)((a, z))$ are true.

Proof. We show simultaneously by induction on $k$ that every $k$ is secured for $a(c)$ and by induction on our priority assignment that each condition $(c, z)$ $((a, z))$ secures at least one $j$ and at most finitely many $j$ for $a(c)$ and that $(c, z)((a, z))$ is true.

The number 0 is secured for $a$ by $(c, 0)$ at stage 0 by definition. Hence, $2 s_{0}^{a}=0$ (Definition 3.5). There is no condition $(a,-1)$ to be satisfied here. At Stage $2,(a, 0)$ secures 0 for $c$ since $(0,0)$ is the only element in $F_{2}^{c}$ and $\{0\}(0)$ diverges under Kleene's indexing; hence, $2 s_{0}^{c}=2$. Moreover, condition $(c, 0)$ is true since there is an $i$ such that $\{0\}(i)$ diverges, namely $i=0$; by Lemma 3.9 it is clear that, for all $s>0, p(s)<_{p}(c, 0)$ since at stage $2,(a, 0)$ secures 0 for $c$. Thus, $(c, 0)$ secures exactly 0 for $a$. (This justifies our ignoring the possibility that $p(2 s)=(c, 0)$ with $s>0$. $)$

This entire paragraph is our inductive hypothesis or consequences of our inductive hypothesis. Suppose that $k$ is the largest number secured for $a$ by condition $(c, z)$ and that condition $(c, z)$ is true. Moreover, we suppose that each number $j$ with $j \leq k$ is secured for $a$ at stage $2 s_{j}^{a}$ where, by Definition 3.5 and Lemmas 3.10 and 3.11, $j<j^{\prime} \leq k$ implies $2 s_{j}^{a}<2 s_{j}^{a}$ and $p\left(2 s_{j}^{a}\right)_{p} \geq p\left(2 s_{j}^{a}\right)_{p} \geq$ $(c, z)$. Moreover, we suppose that each condition $(c, w)$ with $(c, w){ }_{p}>(c, z)$ secures some $j$ for $a$, secures at most finitely many $j$ for $a$, and is true. By Lemma 3.9 and Lemma 3.10, it follows that $p\left(s^{\prime}\right)<_{p}(c, z)$ for all $s^{\prime}>2 s_{k}^{a}$. Suppose that each condition $(a, w)$ with $(a, w) p_{p} \geq(c, z)$ secures some $j$ for $c$, secures at most finitely many $j$ for $c$, and is true. Let $m$ be the largest number secured for $c$ by condition $(a, z-1)$. In addition, we suppose each $j$ with $j \leq m$ is secured for $c$ by some condition $(a, w)$ at stage $2 s_{j}^{c}$; by Definition 3.5 and Lemmas 3.10 and 
3.11, $j<j^{\prime} \leq m$ implies $p\left(2 s_{j}^{c}\right)_{, p} \geq p\left(2 s_{j^{\prime}}^{c}\right)_{p} \geq(a, z-1)$. By Lemma 3.10, $2 s_{m}^{c}<2 s_{k}^{a}$.

Now we will show under the above hypothesis that $(a, z)$ secures $m+1$ for $c$, for some $r(a, z)$ secures $j$ for $c$ iff $m+1 \leq j \leq m+1+r$, and condition $(a, z)$ is true. By the symmetry of the construction it will be clear that one could next establish that $(c, z+1)$ secures $k+1$ for $a$, for some $r(c, z+1)$ secures $j$ for $a$ iff $k+1 \leq j \leq k+1+r$, and condition $(c, z+1)$ is true. From these inductions the results clearly follow.

First we show $(a, z)$ secures $m+1$ for $c$. Since $m$ is the largest number secured for $c$ by $(a, z-1), 2 s_{m}^{c}<2 s_{k}^{a}$, and $p\left(2 s_{k}^{a}\right)=(c, z)$, then by Lemma 3.12, $I_{2 s_{k}^{c}}^{c}(z)=m+1$. By Lemma 3.5 at stage $2 s_{k}^{a}$ all elements of priority higher than $(c, z)$ diverge. Suppose now that either there are no elements of the form $(z, j)$ in $F_{2 s_{k}^{c}}^{c}$ or there is a $(z, j) \in F_{2 s_{k}^{c}}^{c}$ and $\{z\}\left(z_{2 s_{k}^{c}}^{c}\right)$ diverge. In either case, at stage $2 s_{k}^{a}+2$ Case 1 holds, $p\left(2 s_{k}^{a}+2\right)=(a, z)$, and all elements of prioirty higher than $(a, z)$ diverge; conse quently, by Lemma 3.5, $(a, z)$ secures $I_{2 s_{k}^{c}}^{c}(z)=$ $I_{2 s_{k}^{a}}^{c}(z)=m+1$ for $c$ at stage $2 s_{k}^{a}+2$. Suppose next that there is a $(z, j) \epsilon_{2 s_{k}^{c}}^{c}$ and $\{z\}\left(z_{2 s_{k}^{a}}^{c}\right)$ converges; let $2 s_{1}$ be the first stage such that $(E t)_{t \leq 2 s_{1}}$ $T_{1}\left(z, z_{2 s_{k}^{c}}^{c}, t\right)$ and $2 s_{k}^{a}<2 s_{1}$. By Lemma 3.9, $p\left(2 s_{1}-2\right) \leq_{p}(c, z)$. Moreover, $\left(z, z_{2 s_{k}^{c}}^{c}\right) \in F_{2 s_{1}-2}^{c}$ since if $\left(z, z_{2 s_{k}^{c}}^{c}\right)$ is removed from $F_{2 s^{\prime}}^{c}$ where $2 s_{k}^{a}<2 s^{\prime} \leq$ $2 s_{1}-2$ by Lemma $3.1(\mathrm{i})(E t)_{t \leq 2 s^{\prime}} T_{1}\left(z, z_{2 s_{k}^{c}}^{c}, t\right)$, contrary to our choice of $2 s_{1}$. Moreover, $\left(z, z_{2 s_{1}-2}^{c}\right)=\left(z, z_{2 s_{k}^{c}}^{c}\right)$, for otherwise let $2 s^{\prime}$ be the smallest number $2 s$ such that $2 s_{k}^{a}<2 s \leq 2 s_{1}-2$ and $\left(z, z_{2 s}^{c}\right) \neq\left(z, z_{2 s_{k}^{a}}^{c}\right)\left(z_{2 s}^{c}>z_{2 s_{k}^{c}}^{c}\right)$; by Lemma 3.1(ii) $\left(z, z_{2 s^{\prime}}^{c}\right)$ is first placed in $F_{2 s^{\prime}}^{c}$ and by Lemma 3.1(iii) $(E t)_{t \leq 2 s^{\prime}} T_{1}\left(z, z_{2 s_{k}^{c}}^{c}, t\right)$, contrary to our choice of $2 s_{1}$. Thus, Case 2, Subcase B holds at stage $2 s_{1}$ with the $(x, y)$ of Case 2 being $\left(z, z_{2 s_{k}^{c}}^{c}\right)$. Also, either Subcase 1 or Subcase 3 holds at stage $2 s_{1}$, otherwise $p\left(2 s_{1}\right)=(c, z)$ and by Lemmas 3.9 and $3.5,(c, z)$ secures $I_{2 s_{1}}^{a}(z-1)$ for $a$ at stage $2 s_{1}$, contrary to $k$ being the largest element secured for $a$ by $(c, z)$ via Lemma 3.10. By Lemma 3.13, since $\left(z, z_{2 s_{k}^{c}}^{c}\right)$ is removed from $F_{2 s_{1}}^{c}$, there are no elements of the form $(z, j)$ in $F_{2 s_{1}}^{c}$; thus, by Lemma 3.9 since $2 s_{1}>2 s_{k}^{a}$ all elements of priority higher than $(a, z)$ diverge at stage $2 s_{1}$ and by Lemma 3.5 it follows that $(a, z)$ secures $I_{2 s_{1}}^{c}(z)$ for $c$ at stage $2 s_{1}$. We claim that $I_{2 s_{1}}^{c}(z)=I_{2 s_{k}^{a}}^{c}(z)=m+1$ since let $2 s_{0}$ be the 
largest $2 s^{\prime}<2 s_{1}$ such that $p\left(2 s^{\prime}\right)=(c, z)$ and $\left(z, z_{2 s_{k}^{c}}^{c}\right) \in F_{2 s^{\prime}}^{c}$. Clearly, $2 s_{0}=$ $2 s_{k}^{a}$ and following the instructions for Subcase B, Subcase 1 or Subcase 3 let

$$
Q_{c}=\left\{2 s^{\prime}: 2 s_{1}>2 s^{\prime}>2 s_{0} \wedge p\left(2 s^{\prime}\right)=\left(a, x^{\prime}\right)<_{p}(c, z)\right\} \text {. }
$$

If $Q_{c}=\varnothing$, then $2 s_{1}-2=2 s_{k}^{a}$ since $p\left(2 s_{k}^{a}+2\right)=(a, z)$ and, hence, $I_{2 s_{1}}^{c}(z)=$ $r_{2 s_{1}-2}^{c}(z)=I_{2 s_{k}^{a}}^{c}(z)=m+1$. If $Q_{c} \neq \varnothing$, let $2 s_{2}$ be the smallest element of $Q_{c}$. By Lemma 3.4, $p\left(2 s_{2}\right)=(a, z)$ and, hence, $2 s_{2}=2 s_{k}^{a}+2 ; I_{2 s_{1}}^{c}(z)=I_{2 s_{2}}^{c}(z)=$ $m+1$, via Lemma 3.12 since $p\left(2 s_{k}^{a}+1\right)=(a, z)$ and $p\left(2 s_{k}^{a}+2\right)=(a, z)$ implies Case 1 holds at stage $2 s_{k}^{a}+2$ since $2 s_{k}^{a}+2<2 s_{1}$. Thus, $(a, z)$ secures $m+1$ for $c$ at stage $2 s_{1}$.

Next we show that $z \notin D_{2 s_{m+1}^{c}}^{a}$. Suppose $z \in D_{2 s_{m+1}^{a}}^{a}$ and let $s_{0}$ be largest stage $s$ with $s \leq 2 s_{m+1}^{c}$ such that $z$ is placed in $D_{s}^{a}$. By Lemma 3.1(vi) $s_{0}$ is odd and $z$ is placed in $G_{s_{0}}^{a}$. Hence, by Case 1 at odd $s_{0}, p\left(s_{0}-1\right)=(a, z)$. At stage $s_{0}-1,(a, z)$ does not secure $I_{s_{0}-1}^{c}(z)$ for $c$ since otherwise by Lemma 3.10 $2 s_{k}^{a}<s_{0}-1$, and, hence, $(a, z)$ would secure $j$ for $c$ at stage $s_{0}-1$ where $j \geq$ $m+1$, contrary to Lemma 3.11 for $s_{0}-1<2 s_{m+1}^{c}$. By Lemma 3.5 there is some condition $(c, x)((a, x))$ of priority higher than $(a, z)$ such that $\left(x, x_{s_{0}-1}^{c}\right) \epsilon$ $F_{s_{0}-1}^{c}$ and $\{x\}\left(x_{s_{0}-1}^{c}\right)$ converges $\left(\left(x, x_{s_{0}-1}^{a}\right) \in F_{s_{0}-1}^{a}\right.$ and $\{x\}\left(x_{s_{0}-1}^{a}\right)$ converges $)$. At some even stage $2 s$ with $s_{0}-1<2 s \leq 2 s_{m+1}^{c}$, Case 2 holds with the $(x, y)$ of Case 2 having priority higher than $(a, z)$ (since at stage $2 s_{m+1}^{c}$ all elements of priority higher than $(a, z)$ diverge). Since $z \in D_{2 s_{-1}}^{a}$, it follows by Case 2 , Subcase $\mathrm{B}_{2}\left(\mathrm{~A}_{2}\right)$ conditions that $z$ is removed from $D_{2 s}^{a}$, contrary to our choice of $s_{0}$. Thus, at stage $2 s_{m+1}^{c}, z \notin D_{2 s_{m+1}^{c}}^{a}$.

Suppose at stage $2 s_{m+1}^{c}, z \in G_{2 s_{m+1}^{c}}^{a}$ and by the above, $z \notin D_{2 s_{m+1}^{c}}^{a}$. By Lemma 3.2 there are no elements of the form $(z, j)$ in $F_{2 s_{m+1}^{c}}^{a}$ and by Lemma 3.9 it follows that all elements of priority higher than $(c, z+1)$ diverge at stage $2 s_{m+1}^{c}+1$. Thus, by Lemma 3.9, $p\left(2 s^{\prime}\right) \leq_{p}(a, z)$ for all $2 s^{\prime}>2 s_{m+1}^{c}$ and $p\left(2 s^{\prime}\right)$ $<_{p}(a, z)$ for all $2 s^{\prime}>2 s_{m+1}^{c}$ since the only way for $p\left(2 s^{\prime}\right)_{p} \geq(a, z)$ for $2 s^{\prime}>$ $2 s_{m+1}^{c}$, since by Lemma $3.4 p\left(2 s^{\prime}-1\right)<_{p}(a, z)$, is for Case 2 to hold at $2 s^{\prime}$ with the $(x, y)$ of Case 2 having priority higher or the same as $(a, z)$ (this is impossible since all elements of priority higher than $(c, z+1)$ diverge at stage $\left.2 s^{\circ}-1\right)$. Thus, $m+1$ is the only number secured for $c$ by $(a, z)$ and by Lemma $3.2(a, z)$ is true. Thus the inductive step holds.

Suppose that at stage $2 s_{m+1}^{c}$ there is no element of the form $(z, j)$ in $F_{2 s_{m+1}^{a}}^{c}$ 
or there is an element of the form $(z, j)$ in $F_{2 s_{m+1}^{c}}^{a}$ and $\{z\}\left(z_{2 s_{m+1}^{a}}^{c}\right)$ diverges. In the former case, let $2 s$ be the largest stage $2 s^{\prime}$ with $2 s^{\prime} \leq 2 s_{m+1}^{c}$ such that some element $(z, j)$ is removed from $F_{2 s^{\prime}}^{a}$ (2s exists since the first stage $2 s^{\prime}+1$ such that $p\left(2 s^{\prime}+1\right)=(a, z)$ occurs by Lemma 3.4 before stage $\left.2 s_{m+1}^{c}\right)$. By Lemma 3.1(i), $z \in G_{2 s}^{a}$ and by Lemma 3.1(vii), it follows that $z \in G_{2 s_{m+1}^{c}}^{a}$. However, this case was treated in the paragraph immediately above. In the latter case at stage $2 s_{m+1}^{c}+1$ all conditions of priority higher than $(c, z+1)$ diverge; thus, as above, $p\left(s^{\prime}\right) \leq_{p}(c, z+1)$ for all $s^{\prime}>2 s_{m+1}^{c}$. Hence, $m+1$ is the only number secured for $c$ by $(a, z)$ and, clearly, in either case $(a, z)$ is true. Thus, the result holds.

We claim that $(* *)$ for every $2 s \geq 2 s_{m+1}^{c}$ such that $p(2 s)=(a, z)$ and $r=$ $I_{2 s}^{c}(z)$ that every number $j, m+1 \leq j \leq r$, is secured for $c$ by $(a, z)$ at stage $2 s_{j}^{c} \leq 2 s$. Clearly, the result holds for $2 s=2 s_{m+1}^{c}$ and suppose the result holds for all $2 s^{\prime}, 2 s_{m+1}^{c} \leq 2 s^{\prime}<2 s$, where $p(2 s)=(a, z)$. By Lemma 3.9, $p(2 s-2) \leq_{p}$ $(a, z)$ and by Lemma $3.4, p(2 s-1)<_{p}(a, z)$. Thus, at stage $2 s$, Case 2 holds and necessarily the element $(x, y)$ of Case 2 is $\left(z, z_{2 s-1}^{a}\right)$ since at stage $2 s-1$ all elements of priority higher than $(a, z)$ diverge at Lemma 3.9 and $p(2 s)=$ $(a, z)$. Moreover, Subcase 2 holds at stage $2 s$ since $p(2 s)=(a, z)$; thus, $\{z\}\left(z_{2 s-1}^{a}\right) \geq z_{2 s-1}^{a}+1$. Let $2 s_{0}$ be the largest stage $2 s^{\prime}<2 s$ such that $p\left(2 s^{\prime}\right)=$ $(a, z)$ and $\left(z, z_{2 s-1}^{a}\right) \in F_{2 s^{\prime}}^{a}$. It follows that $2 s_{0} \geq 2 s_{m+1}^{c}$ since if $\left(z, z_{2 s-1}^{a}\right) \epsilon$ $F_{2 s_{m+1}^{c}}^{a}$, this is clear and $\left(z, z_{2 s-1}^{a}\right)$ is first placed in $F_{2 s^{\prime \prime}}^{a}$ where by Lemma 3.3, $2 s^{\prime \prime}>2 s_{m+1}^{c}$ if $\left(z, z_{2 s-1}^{a}\right) \notin F_{2 s_{m+1}^{c}}^{a}$ (note that if $2 s_{m+1}^{c}<2 s^{\prime \prime}$, then $2 s_{m+1}^{c}<$ $2 s_{0}$, and since $p\left(2 s_{0}\right)=(a, z)$, Case 2 , Subcase 2 holds at stage $2 s_{0}$ but then by Lemma 3.3 and Case 2, Subcase 2 procedures it follows that $\left(z, z_{2 s-1}^{a}\right)$ is first placed in $F_{2 s_{0}}^{a}$, i.e., $\left.2 s^{\prime \prime}=2 s_{0}\right)$. An element $(z, j)$ is first placed in $F_{2 s}^{a}$ and by Lemmas 3.9 and $3.5,(a, z)$ secures $I_{2 s_{0}}^{c}(z)+1$ at stage $2 s$ since by the construction $I_{2 s}^{c}(z)=I_{2 s_{0}}^{c}(z)+1$. By our hypothesis at $2 s_{0}$, the result follows.

Suppose now that at stage $2 s_{m+1}^{c}$ there is an element of the form $(z, j)$ in $F_{2 s_{m+1}^{c}}^{a},\{z\}\left(z_{2 s_{m+1}^{a}}^{c}\right)$ converges, and $\{z\}\left(z_{2 s_{m+1}^{a}}^{c}\right) \leq z_{2 s_{m+1}^{a}}^{a}$; or some element $\left(z, j^{*}\right)$ is first placed in $F_{2 s^{\prime}}^{a}$ for some $2 s^{\prime}>2 s_{m+1}^{c}$ and $\{z\}\left(j^{*}\right) \leq j^{*}$. In either case it is clear that condition $(a, z)$ is true and that at some stage $2 s_{0}^{\prime}>2 s_{m+1}^{c}$, Case 2 , Subcase 1 or Subcase 3 holds at stage $2 s_{0}^{\prime}$ with the $(x, y)$ of Case 2 being $\left(z, z_{2 s_{m+1}^{c}}^{a}\right)\left(\left(z, j^{*}\right)\right)$. At stage $2 s_{0}^{j}$ all elements of the form $(z, j)$ in $F_{2 s_{0}^{\prime}-1}^{a}$ are removed from $F_{2 s_{0}}^{a}$. Consequently, all elements of priority higher 
than $(c, z+1)$ diverge at stage $2 s_{0}^{\prime}$ and, hence, for all $2 s^{\prime} \geq 2 s_{0}^{\prime}, p\left(2 s^{\prime}\right)<_{p}$ $(a, z)$. Thus, $(a, z)$ secures only finitely many $j$ for $c$ and by $(* *)$ above there is some $r$ such that $(a, z)$ secures $j$ for $c$ iff $m+1 \leq j \leq m+1+r$.

Similarly, if at some stage $2 s^{\prime}>2 s_{m+1}^{c}$ an element $\left(z, j^{*}\right)$ such that $\{z\}\left(j^{*}\right)$ diverges is first placed in $F_{2 s^{\prime}}^{a}$, then all elements of priority higher than $(c, z+1)$ diverge at stage $2 s^{\prime}+1$ and hence $p(2 s)<_{p}(a, z)$ for all $2 s>2 s^{\prime}$. Hence, $(a, z)$ secures only finitely many $j$ for $c$ and by $(* *)$ at $2 s^{\prime}$, there is an $r$ such that $(a, z)$ secures $j$ for $c$ iff $m+1 \leq j \leq m+1+r$.

We may now suppose that every element $(z, j)$ which is placed in $F_{2 s}^{a}$ has the property that $\{z\}(j)$ converges and $\{z\}(j) \geq j+1$. Moreover, we can suppose $z \notin G_{2 s_{m+1}^{a}}^{c}\left(z \notin D_{2 s_{m+1}^{a}}^{c}\right.$ is established above). We assume these additional hypotheses for the remaining part of the proof of this lemma; otherwise, we are done by the above argument.

Suppose $z$ is placed in $G_{s}^{a}$ at some stage $s$ with $s>2 s_{m+1}^{c}$. Clearly, $s$ cannot be even for if so, at stage $s$ Case 2, Subcase A, Subcase 1 holds with the $(x, y)$ of Case 2 being $\left(z, z_{s-1}^{a}\right) \in F_{s-1}^{a}$; but then $\{z\}\left(z_{s-1}^{a}\right)<z_{s-1}^{a}+1$ contrary to the above hypothesis. Thus, $s$ is odd and we claim $(a, z)$ is true and that the other results of our inductive step are true. First, replace $s$ by $2 s+1$; then clearly Case 1 holds for $(a, z)$ at stage $2 s+1$. By Case 1 conditions at stage $2 s+1$, there are two elements $\left(z, k_{1}\right),\left(z, k_{2}\right)$ in $F_{2 s}^{a}$ with $k_{1}<k_{2}$ such that Case 1 holds for $\left(z, k_{1}\right)$ and $\left(z, k_{2}\right)$ at stage $2 s+1$, i.e., the following conditions are true: First using the same notation as in stage $2 s+1$, Case 1 , let $2 s_{0}$ be the largest $2 s^{\prime}<2 s$ such that $p\left(2 s^{\prime}\right)=(c, z)$; clearly by our inductive hypothesis since $2 s+1>2 s_{m+1}^{c}>2 s_{k}^{a}, 2 s_{0}=2 s_{k}^{a}$. Let $2 s_{1}$ be the largest stage $2 s^{\prime}$ such that $2 s_{0} \leq 2 s^{\prime} \leq 2 s$ and for some $j(z, j)$ is removed from $F_{2 s^{\prime}}^{c}$ or $2 s_{1}=2 s_{0}$, otherwise. Clearly, $2 s_{1} \leq 2 s_{m+1}^{c}$ since at stage $2 s_{m+1}^{c}$ all elements of priority higher than $(a, z)$ diverge and by Lemma 3.9 no element of the form $(z, j)$ is removed from $F_{s^{\prime}}^{c}$ where $s^{\prime}>2 s_{m+1}^{c}$. If $2 s_{1}>2 s_{0}$, it follows that $2 s_{1}=2 s_{m+1}^{c}$ since by Lemma 3.13 at stage $2 s_{1}$ all elements of the form $(z, j)$ are removed from $F_{2 s_{1}}^{c}$ and consequently so is $\left(z, z_{2 s_{1}-1}^{c}\right)$. By Lemmas 3.1(iii) and 3.3, stage $2 s_{1}$ is the first stage larger than $2 s_{k}^{a}$ such that all elements of priority higher than $(a, z)$ diverge and by Case 2, Subcase B, Subcase 1 or Subcase 3 conditions $p\left(2 s_{1}\right)=(a, z)$. Now let $j^{*}$ and $2 s_{2}$ be as in Case 1 , stage $2 s+1$. Hence, $f^{a}\left(2 s_{0}\right) \leq j^{*}$ and for $2 s_{2}, 2 s_{1} \leq 2 s_{2} \leq 2 s, p\left(2 s_{2}\right)=(a, z)$ and $j^{*}=I_{2 s_{2}}^{c}(z)$. Clearly, $2 s_{m+1}^{c} \leq 2 s_{2}$ since $2 s_{m+1}^{c}$ equals the smallest stage $2 s^{\prime}$ such that $2 s^{\prime} \geq$ $2 s$, and $p\left(2 s^{\prime}\right)=(a, z)$ from the above. By $(* *),(a, z)$ secures each $j$ with $m+1 \leq$ $j \leq j^{*}$ for $c$ at stage $2 s_{j}^{c}$ and by our inductive hypothesis each $j$ with $j<m+1$ is 
secured for $c$ at stage $2 s_{j}^{c}$. Thus, by Lemma 3.5, $\left|\gamma_{f} c\left(2 s_{j}^{c}\right)\right|=\left|\delta\left(b_{j-1}, b_{j}\right)\right|$ for each $j \leq j^{*}$ and by Lemma 3.11, $j<j^{\prime} \leq j^{*}$ implies $2 s_{j}^{c}<2 s_{j^{\prime}}^{c} \leq 2 s_{j}^{c}=2 s_{2}$. Thus,

$$
\left|c_{f^{c}\left(2 s_{2}\right)}\right|=\left|\sum_{i=0}^{f^{c}\left(2 s_{2}\right)} \gamma_{i}\right| \geq\left|\sum_{i=0}^{j^{*}} \delta\left(b_{j-1}, b_{j}\right)\right|=\left|b_{j}\right| \text {. }
$$

By Lemma 3.8,

$$
\left|a_{f_{\left(2 s_{0}\right)}}\right| \leq\left|b_{f_{\left(2 s_{0}\right)}}\right| \leq\left|b_{j}\right|
$$

Thus, $\left|a, a_{\left(2 s_{0}\right)}\right| \leq\left|c_{f} a_{\left(2 s_{2}\right)}\right|$. But $f^{c}\left(2 s_{2}\right) \leq k_{1}$ and, consequently, $\left|c_{f} c_{\left(2 s_{2}\right)}\right| \leq$ $\left|c_{k_{1}}\right|$. Now we claim condition $(a, z)$ is true. Suppose that $\left.\left|c_{k_{1}}\right| \leq \mid a_{\{z}\right\}\left(k_{1}\right) \mid$ (by Lemma 3.1(iii) we know $\{z\}\left(k_{1}\right)$ converges since $\left(z, k_{2}\right)$ with $k_{2}>k_{1}$ is first placed in $F_{s^{\prime}}^{a}$ at stage $s^{\prime}$ later than stage $s^{\prime \prime}$ where $\left(z, k_{1}\right)$ is first placed in $\left.F_{s^{\prime \prime}}^{a}\right)$. Consequently, by the above the following inequality is true:

$$
\left|a_{f^{a}\left(2 s_{0}\right)}\right| \leq\left|c_{f^{c}\left(2 s_{2}\right)}\right| \leq\left|c_{k_{1}}\right| \leq\left|a_{\{z\}\left(k_{1}\right)}\right| \leq\left|a_{k_{2}}\right| .
$$

Thus, $\left|\delta\left(a_{f} a_{\left(2 s_{0}\right)}, a_{k_{2}}\right)\right| \geq\left|\delta\left(c_{f} c_{\left(2 s_{2}\right)}, c_{k_{1}}\right)\right|$. Howerer, by Case 1, stage $2 s+1$ conditions, it follows that

$$
\left|\delta\left(c_{f}{ }^{\left(2 s_{2}\right)}, c_{k_{1}}\right)\right| \geq \omega \cdot m^{*}, \quad\left|\delta\left(a_{f^{a}\left(2 s_{0}\right)}, a_{k_{2}}\right)\right| \leq \omega \cdot m, \text { and } m^{*}>m \text {, }
$$

a contradiction. Thus, it follows that $\left|a_{\{z\}\left(k_{1}\right)}\right|<\left|c_{k_{1}}\right|$; therefore, condition $(a, z)$ is true. Applying result $(* *)$ above at stage $2 s \geq 2 s_{m+1}^{c}$, it follows that $(a, z)$ secures all numbers $j$ for $c$ with $m+1 \leq j \leq I_{2 s}^{c}(z)$. At stage $2 s^{\prime}$ where $2 s^{\prime}$ is the smallest number $2 s^{\prime \prime} \geq 2 s+1$ such that $(E t)_{t \leq 2 s^{\prime \prime}} T_{1}\left(z, z_{2 s+1}^{a}, t\right)$

Case 2, Subcase $A$, Subcase 3 holds since $z \in G_{2 s^{\prime}-1}^{a}$; hence, $p\left(2 s^{\prime}\right)=(c, z+1)$ and at stage $2 s^{\prime}$ all elements of priority higher than $(c, z+1)$ diverge. By Lemma 3.9, for all $s^{\prime \prime}>2 s^{\prime}, p\left(s^{\prime \prime}\right) \leq_{p}(c, z+1)$ and, thus, $I_{2 s}^{c}(z)$ is the largest number secured for $c$ by $(a, z)$. This completes the induction in case $z$ is placed in $G_{2 s+1}^{a}$ at some stage $2 s+1>2 s_{m+1}^{c}$.

It remains to be shown under the above hypotheses that eventually at some stage $2 s+1$ with $2 s+1>2 s_{m+1}^{c}$ that Case 1 holds for $(a, z)$. Suppose that Case 1 never holds for $(a, z)$ at any stage $2 s+1>2 s_{m+1}^{c}$. Let $j_{m+1}=z_{2 s_{m+1}^{c}}^{c}$ since by the above subsidiary hypothesis there is some $j$ such that $(z, j) \epsilon$ $F_{2 s_{m+1}^{c}}^{a}$; moreover, by this hypothesis $\{z\}\left(j_{m+1}\right)$ converges and $\{z\}\left(j_{m+1}\right)>j_{m+1}$. 
Let $2 s_{1}^{\prime}$ be the smallest number $2 s>2 s_{m+1}^{c}$ such that $(E t)_{t \leq 2 s} T_{1}\left(z, j_{m+1}, t\right)$. By Lemma 3.9 for all $s$ with $s \geq 2 s_{m+1}^{c}, p(s) \leq_{p}(a, z)$ and by our choice of $2 s_{1}^{\prime}$, $p(s)<_{p}(a, z)$ for $2 s_{m+1}^{c}<s<2 s_{1}^{\prime}$. By Lemma 3.1(i), $\left(z, j_{m+1}\right) \in F_{s}^{a}$ for each $s$ with $2 s_{m}^{c} \leq s<2 s_{1}^{\prime}$; in particular $\left(z, j_{m+1}\right) \in F_{2 s_{1}^{\prime}-1}^{a}$ and $\left(z, j_{m+1}\right)=\left(z, z_{2 s_{1}^{\prime}-1}^{a}\right)$. Also $z \notin G_{2 s_{1}^{\prime}-1}^{a}$ since $z \in G_{2 s_{1}^{\prime}-1}^{a}$ implies $z$ is placed in $G_{s}^{a}$ for some $s$ with $2 s_{m}^{c}<s \leq 2 s_{1}^{\prime}-1$ but $s$ is odd implies Case 1 holds for $(a, z)$ at stage $2 s+1$, contrary to our assumption, and $s$ is even implies that Case 2, Subcase A, Subcase 1 holds at stage $s$ with $\left(z, z_{s-1}^{a}\right)=\left(z, j_{m+1}\right)$ being the $(x, y)$ of the construction, contrary to our choice of $2 s_{1}^{\prime}$ (and $\{z\}\left(j_{m+1}\right)>j_{m+1}$ ). Thus, at stage $2 s_{1}^{\prime}$ Case 2, Subcase $A$, Subcase 2 holds with the $(x, y)$ of the construction being $\left(z, j_{m+1}\right)$. At stage $2 s_{1}^{\prime}, p\left(2 s_{1}^{\prime}\right)=(a, z)$ and by Lemmas 3.9 and $3.5,(a, z)$ secures $I_{2 s_{1}^{\prime}}^{c}(z)$ for $c$; however, by Subcase $A$, Subcase $2, I_{2 s_{1}^{\prime}}^{c}(z)=I_{2 s_{m+1}^{c}}^{c}(z)+$ $1=m+2$ since by the above $p(s)<_{p}(a, z)$ for every $s$ with $2 s_{m+1}^{c}<s<2 s_{1}^{\prime}$, i.e., $2 s_{m+1}^{c}$ is the largest number $2 s^{\prime}<2 s_{1}^{\prime}$ such that $p\left(2 s^{\prime}\right)=(a, z)$ and $\left(z, j_{m+1}\right) \epsilon$ $F_{2 s^{\prime}}^{a}$. Thus, $2 s_{1}^{\prime}=2 s_{m+2}^{c}$ and $z \notin G_{2 s_{m+2}^{a}}^{a}$. At stage $2 s_{1}^{\prime}$, an element $\left(z, j_{m+2}\right)$ is first placed in $F_{2 s_{m+2}^{c}}^{a}$ and clearly $j_{m+1}^{m+2}<\{z\}\left(j_{m+1}\right)<j_{m+2}$ since by Kleene's Gödel numbering $T_{1}\left(z, j_{m+1}, t\right)$ implies $\{z\}\left(j_{m+1}\right)<t$ and $t \leq j_{m+2}$. Thus, at stage $2 s_{m+2}^{c}$ by our subsidiary hypothesis, exactly the same circumstances hold as at stage $2 s_{m+1}^{c}$. Thus by induction on this argument for each $n \geq m+1$ there is a stage $2 s_{n}^{c}$ such that $(a, z)$ secures $n$ for $c$ at stage $2 s_{n}^{c}$; moreover, at stage $2 s_{n}^{c}$ for $n>m+1$ an element $\left(z, j_{n}\right)$ is first placed in $F_{2 s_{n}^{c}}^{c^{n}}$ and for all $n \geq m+1$, $j_{n}<\{z\}\left(j_{n}\right)<j_{n+1}$. By Lemma 3.11, $2 s_{n}^{c}<2 s_{n+1}^{c}$ for all $n$. Now we will show that for sufficiently large $2 s+1$, at stage $2 s+1$, Case 1 holds for $(a, z)$, contrary to our assumption. For $2 s+1$ with $2 s+1>2 s_{m+1}^{c}$, let $2 s_{0}$ equal the largest stage $2 s^{\prime}<2 s+1$ such that $p(2 s)=(c, z)$; by our inductive hypothesis, $2 s_{0}=2 s_{k}^{a}$. Also, let $2 s_{1}$ equal the largest stage $2 s^{\prime}<2 s+1$ such that for some $j(z, j)$ is removed from $F_{2 s^{\circ}}^{c}$. By Lemma 3.9, $2 s_{1} \leq 2 s_{m+1}^{c}$ and as in the preceding paragraph $2 s_{m+1}^{c}$ equals the first stage $2 s^{\prime} \geq 2 s_{1}$ such that $p\left(2 s^{\prime}\right)=(a, z)$. Compute $f^{a}\left(2 s_{0}\right)$ and let $2 s_{2}^{\prime}=2 s_{m+1}^{c}$ if $m+1 \geq f^{a}\left(2 s_{0}\right)$ or $2 s_{2}^{\prime}=2 s^{c} f^{a}\left(2 s_{0}\right)$, if $m+1<$ $f^{a}\left(2 s_{0}\right)$. It is clear that for $2 s_{j}^{c}+1>2 s_{2}^{\prime}\left(p\left(2 s_{j}^{c}\right)=(a, z)\right)$ that the $j^{*}$ and $2 s_{2}$ chosen as in the procedure for Case 1 at stage $2 s_{j}^{c}+1$ are always $I_{2 s}^{c},(z)$ and $2 s_{2}^{\prime}$, respectively. (Note that $(*)$ of Case 1 , stage $2 s_{j}^{c}+1$ with $2 s_{j}^{c}+1>2 s_{2}^{\prime}$ holds for all $t$ since $(c, z)$ secures $k$ for $a$ at stage $2 s_{0}$ and $(a, z)$ secures $I_{2 s_{2}^{\prime}}^{c}(z)$ for $c$ at stage $2 s_{2}^{\prime}$ via Definition 3.3.) Let $i^{*}$ be the first $i>I_{2 s_{2}^{\prime}}^{c}(z) \geq$ 
$f^{a}\left(2 s_{0}\right)$ such that $\left|\delta\left(b_{i-1}, b_{i}\right)\right| \geq \omega^{2}$ since by the hypothesis of Theorem 3.1 such an $i$ always exists [0]. Let $i^{*}$ be secured for $c$ at stage $2 s_{i}^{c}$ where $i^{*}>m+1$ since $I_{2 s}^{c}(z) \geq m+1$. By the above there are infinitely many $j_{n}^{\prime}$ 's such that $\left(z, j_{n}\right)$ is placed in $F_{2 s_{n}^{c}}^{a}$ and $j_{n}<\{z\}\left(j_{n}\right)<j_{n+1}$, choose $k_{1}$ equal to the smallest $j_{n}$ such that $j_{n} \geq f^{c}\left(2 s_{i}^{c} c^{n}\right)$. By the construction, $\left|c_{f} f_{\left(2 s_{i}^{*}\right)}\right| \leq\left|c_{k_{1}}\right|$ and by Lemma 3.8 ,

$$
\left|a_{f\left(2 s_{0}\right)}\right| \leq\left|b_{f\left(2 s_{0}\right)}\right|
$$

By Lemma 3.11, $2 s_{0}^{c}<2 s_{1}^{c}<\cdots<2 s_{i}^{c}$ and hence by Lemma 3.5

$$
\left|b_{f_{\left(2 s_{0}\right)}^{a}}\right| \leq\left|c_{f^{c}\left(2 s_{2}^{\prime}\right)}\right| \leq\left|c_{f^{c}\left(2 s_{i} c^{*}\right)}\right|
$$

since $2 s_{2}^{\prime} \geq 2 s_{f}^{c}{ }^{a}\left(2 s_{0}\right)^{\circ}$ Clearly, $\left|\delta\left(c_{f} c_{\left(2 s_{2}^{\prime}\right)}, c_{k_{1}}\right)\right| \geq \omega^{2}$ since $\left|\delta\left(c_{f} c_{\left(2 s_{2}^{\prime}\right)}, c_{k_{1}}\right)\right| \geq$ $\left|\gamma_{f^{c}\left(2 s_{i}^{c} c^{c}\right.}\right| \geq \omega^{2}$. By Theorem 1.1, let $m_{2 s+1}^{*}=$ the number of distinct limit notations in enm $(d, 0), \cdots, \operatorname{enm}(d, 2 s+1)$ where $d=\delta\left(c_{f}{ }^{c}\left(2 s_{2}^{\prime}\right), c_{k_{1}}\right)$; clearly, $m_{2 s+1}^{*}$ is an unbounded nondecreasing function of $2 s+1$. Let $s^{*}=\left\{2 s^{\prime}: 2 s_{0}<2 s^{\prime}\right.$, $p\left(2 s^{\prime}\right)=(c, w)$, and $\left.f^{a}\left(2 s^{\prime}\right) \leq k_{2}\right\}$ where $k_{2}$ is the first $j_{n}>k_{1}$. Since $f^{a}$ is increasing, it is clear that $s^{*}$ is finite. For $2 s^{\prime} \in s^{*}$ since $2 s_{k}^{a}=2 s_{0}<2 s^{\prime}$, $p\left(2 s^{\prime}\right)=(c, w)<_{p}(a, z)$. By Lemma 3.10, for any $2 s^{\prime} \in S^{*} p\left(2 s^{\prime}\right)=(c, w)$ does not secure $I_{2 s^{\prime}}^{a}(w-1)$ for $a$ at stage $2 s^{\prime}$, for otherwise $2 s^{\prime}>2 s_{n}^{c}$ for all $n \geq m+$ 1. Thus, by Definition 3.3 for each $2 s^{\prime} \in S^{*}$, there is a smallest number $t_{2 s^{\prime}}$ such that $\left\{r\left(\left(p\left(2 s^{\prime}\right), 2 s^{\prime}\right)\right)\right\}\left(t_{2 s^{\prime}}\right) \neq 0$. For each $2 s^{\prime} \in s^{*}$ let $q_{2 s^{\prime}}$ be obtained via Theorem 2.2(vi) and $t_{2 s^{\prime}}$ so that $\left|\alpha_{f^{a}\left(2 s^{\prime}\right)}\right| \leq \omega \cdot q_{2 s^{\prime}}$. Let $m=\left(\sum_{2 s^{\prime} \in s^{*}} q_{2 s^{\prime}}\right)+1$. Finally, choose $2 s_{n}^{c}+1$ with $2 s_{n}^{c}+1>2 s^{c}{ }^{*}+1$ so large that $\left(z, k_{1}\right),\left(z, k_{2}\right) \epsilon$ $F_{2 s_{n}^{c}}^{a}$ (Lemma 3.1(iii)), $2 s_{n}^{c}+1>2 s_{2}^{\prime}, m_{2 s_{n}^{c} *^{i}}^{c}>m, k_{2} \leq f^{a}\left(2 s_{n}^{c}\right)$, and for each $t_{2 s^{\prime}}$ with $2 s^{\prime} \in S^{*}, t_{2 s^{\prime}} \leq 2 s_{n}^{c}+1$. At stage $2 s_{n}^{c}+1$ under Case 1 procedure for the above choice of $k_{1}, k_{2}\left(k_{1}<k_{2}\right)$ and $\left(z, k_{1}\right),\left(z, k_{2}\right)$ in $F_{2 s_{n}}^{a} j^{*} j^{*}$ and $2 s_{2}$ are respectively $I_{2 s_{2}^{\prime}}^{c}(z)$ and $2 s_{2}^{\prime}$ above, $S$ is $S^{*}$ above, $m^{*}$ is $m_{2 s_{n}^{c}}^{*}{ }_{n}^{2}$ above, and $m$ is $m$ above. Hence, at stage $2 s_{n}^{c}+1$ Case 1 holds for $(a, z)$, a contradiction. Thus, under the above hypotheses, there is always some stage $2 s+1>2 s_{m+1}^{c}$ such that Case 1 holds for $(a, z)$ at stage $2 s+1$. By the preceding paragraph the inductive step follows. Q.E.D. 
Now by virtue of Lemma 3.14 all conditions $(2)_{x}^{a}$ and $(2)_{x}^{c}$ are true and in view of Lemmas 3.11 and 3.14 all conditions $(3)_{n}^{a}$ and $(3)_{n}^{c}$ are true. By Lemma 3.8, conditions $(1)_{s}^{a}$ and $(1)_{s}^{c}$ are true. Thus, Theorem 3.1 is established.

Theorem 3.2. If $\omega^{3} \leq \gamma<\omega_{1}$ and $\gamma$ is not of the form $\alpha+1, \alpha+\omega$, or $\alpha+\omega^{2}$ for any ordinal $a$, then $\mathcal{L}(\gamma)$ bas no minimal elements and below any element of $\mathcal{L}(y)$ are two elements of incomparable many-one degrees.

Proof. Immediate by Theorem 3.1 and Theorem 1.6 since, for some $\alpha, \beta$, $\gamma=\alpha+\beta$ such that $\beta \geq \omega^{3}$ and $\beta$ is principal for addition [0].

\section{REFERENCES}

0. Alexander Abian, The theory of sets and transfinite arithmetic, Saunders, Philadelphia and London, 1965.

1. Martin Davis, On the theory of recursive unsolvability, Ph.D. thesis, Princeton University, Princeton, N. J., 1950.

2. - Relatively recursive functions and the extended Kle ene hierarchy, Proc. Internat. Congress Math., 1952, vol. 1, p. 723.

3. S. C. Kleene, On the forms of the predicates in the theory of constructive ordinals, Amer. J. Math. 66 (1944), 41-58. MR 5, 197.

4. —, Introduction to metamathematics, Van Nostrand, Princeton, N. J., 1952. MR 14, 525.

5. - Arithmetical predicates and function qualifiers, Trans. Amer. Math. Soc. 79 (1955), 312-340. MR 17, 4.

6. - On the forms of the predicates in the theory of constructive ordinals. II, Amer. J. Math. 77 (1955), 405-428. MR 17, 5.

7. S. C. Kleene and E. L. Post, The upper semi-lattice of degrees of recursive unsolvability, Ann. of Math. (2) 59 (1954), 379-407. MR 15, 772.

8. Y. N. Moschovakis, Many-one degrees of the predicates $H_{a}(x)$, Pacific J. Math. 18 (1966), 329-342. MR 37 \#1247.

9. A. Mostowski, Über gewisse universelle Relationen, Ann. Soc. Polon. Math. 17 (1938), 117-118.

10. H. Rogers, Jr., Theory of recursive functions and effective computability, McGraw-Hill, New York, 1967. MR 37 \#61.

11. E. L. Post, Recursively enumerable sets of positive integers and their decision problems, Bull. Amer. Math. Soc. 50 (1944), 284-316. MR 6, 29.

12. G. E. Sacks, Degrees of unsolvability, Princeton Uni v. Press, Princeton, N. J., 1963. MR $32 \# 4013$.

13. C. Spector, Recursive well-orderings, J. Symbolic Logic 20 (1955), 151-163. MR 17, 570, 1437.

DEPARTMENT OF MATHEMATICS, UNIVERSITY OF IOWA, IOWA CITY, IOWA $\$ 2240$ 